\title{
Attitude determination of planetary exploration rovers using solar panels characteristics and accelerometer
}

Takayuki Ishida $^{1 \dagger}$, Masaki Takahashi ${ }^{2}$

${ }^{1}$ School of Science for Open and Environment Systems, Graduate School of Science and Technology, Keio University, 3-14-1 Hiyoshi, Kohoku-ku, Yokohama 223-8522, Japan, Tel.:+81 45566 1660; Fax: +81 455661660 .

${ }^{2}$ Department of System Design Engineering, Keio University, 3-14-1 Hiyoshi, Kohoku-ku, Yokohama 223-8522, Japan

${ }^{\dagger}$ Corresponding author

Email addresses:

Takayuki Ishida: apoptoxin4869@z3.keio.jp

Masaki Takahashi: takahashi@sd.keio.ac.jp 


\section{Abstract}

In this study, we propose a new attitude determination system, which we call Irradiance-based Attitude Determination (IRAD). IRAD employs the characteristics and geometry of solar panels. First, the sun vector is estimated using data from solar panels including current, voltage, temperature, and the normal vectors of each solar panel. Because these values are obtained using internal sensors, it is easy for rovers to provide redundancy for IRAD. The normal vectors are used to apply to various shapes of rovers. Second, using the gravity vector obtained from an accelerometer, the attitude of a rover is estimated using a three-axis attitude determination method. The effectiveness of IRAD is verified through numerical simulations and experiments that show IRAD can estimate all the attitude angles (roll, pitch, and yaw) within a few degrees of accuracy, which is adequate for planetary explorations.

\section{Keywords}

Planetary rover

Navigation

Attitude determination

Solar panel

$q$ method 


\section{Introduction}

Planetary rovers are being increasingly used in space exploration missions. With their ability to traverse the surface of planets they are capable of in-situ exploration even in areas where landers cannot land, such as steep craters. The National Aeronautics and Space Administration (NASA) sent two rovers, named Mars Exploration Rovers (MER) Spirit and Opportunity, to Mars in 2003 [1]. The two rovers subsequently explored the surface of Mars and gathered a wealth of information. In 2011 NASA also sent Curiosity to explore the habitability of Mars [2]. The Japan Aerospace Exploration Agency (JAXA) is currently planning a mission to the moon called the SELENE-2 mission [3]. In this mission, rovers are expected to travel across wide open areas and observe terrain features using onboard scientific instruments.

In planetary exploration missions using rovers, navigation relies on integrated local measurements, such as combinations of inertial sensors, and wheel and visual odometry. These systems are essential for obtaining data on the attitude of rovers that are required for accurate guidance. This system for planetary rovers is called an attitude determination system.

A number of studies have proposed attitude determination systems for rovers. The Mars exploration rovers, Spirit and Opportunity [4], explored the surface of Mars and gathered a wealth of information using an attitude determination system with cameras and inertial sensors. Furgale et al. [5] proposed a system that combined a sun sensor and an accelerometer, and showed its efficiency in experiments.

For future missions using rovers, a redundant attitude determination system is now a requirement. First, because rovers are required to enable long traverse 
explorations and long mission terms. Additionally a sensor malfunction was reported in the Curiosity mission [6]. A redundant attitude determination system is needed in such cases. Generally there are two means of building a redundant system, one is to equip with identical redundant sensors, and the other is to use a different combination of sensors. Therefore in this study, we aim at building an attitude determination system by the different combination of sensors that rovers are already equipped with. In this study, we propose a new attitude determination system, which we call Irradiance-based Attitude Determination (IRAD). IRAD combines the characteristics of solar panels, and an accelerometer. The sun vector can be estimated by measuring the current, voltage, and temperature of the solar panels as the electric power generation of each solar panel depends on their arrangement and the position of the sun. By combining this sun vector with the gravity vector obtained using an accelerometer, the attitude of the rover can be estimated. The $q$ method [7] is generally known as a technique for obtaining an attitude from two observation vectors. Santoni et al. [8][9] proposed an attitude determination system for small spinning spacecraft, which combines the characteristics of solar panels with a magnetometer. This system was aimed at regular octagonal pillar shaped spacecraft, which is a characteristic shape of spinning spacecraft. Burton et al. [10] proposed an online attitude determination filter using the electrical current from body mounted solar panels and the spacecraft dynamics for the CubeSat nano-satellites. In this study we employ the minimization of a cost function using the normal vectors of the solar panels of the rovers to apply to various shapes of rovers. The efficiency of IRAD was validated through numerical simulations and experiments. 
IRAD is useful for planetary rovers. First, it does not require any external sensors. Second, the solar panels necessary for power generation can also be used as sensors for attitude determination. 


\section{Mathematical models}

This chapter provides the mathematical models for the analysis used in this study. We first define the frames of reference and mathematical models for solar panels and noise which has an effect upon an attitude determination system. These noises should be considered and compensated to estimate the attitude precisely.

\subsection{Frames of reference}

As shown in Fig. 1a, the topocentric frame is defined with respect to the local horizon, the $Z_{t}$ axis is the normal vector to the tangent plane, the $Y_{t}$ axis is along North and the $X_{t}$ axis is defined by the right-handed system. In contrast, as shown in Fig. $1 b$, the origin of the rover frame is located at the rover itself, the $X_{r}$ axis is along the direction of travel, the $Z_{r}$ axis is vertical to the rover and the $Y_{r}$ axis is defined by the right-handed system. We define rotation around the $X_{r}$ axis as roll, the $Y_{r}$ axis as pitch, and the $Z_{r}$ axis as yaw.

\subsection{Solar panels model}

IRAD employs the electric power generated by the solar panels to estimate an attitude of the rover. Therefore solar panel modeling is required as discussed in this section.

There is relationship between the voltage and current of a solar panel, which is called the $I-V$ curve. It is possible to store the $I-V$ curve in the memory of a rover, but 
it varies depending on the irradiance and temperature of the solar panel, so it is not realistic to store all the $I-V$ curves corresponding to possible irradiance and temperature conditions. Therefore, solar panel modeling is required to obtain the $I-V$ curve from sensor values and the ephemeris, for example voltage, temperature and irradiance.

Several solar panel models for silicon solar cell have been proposed as equivalent circuits [11-13]. In this study we use the two-diode model [13]. Fig. 2 shows the equivalent circuit describing the two-diode model. $I_{P V}$ is the light generate current, $I_{D 1}$ and $I_{D 2}$ are the reverse saturation currents of diode, $R_{s}$ and $R_{p}$ are series resistance and shunt resistance respectively, and $I$ is output current.

Although we used silicon solar cells in this study because of their availability, triple-junction solar cells have been used in recent space missions. In this case, the model [14] is different from that used in this study. It is necessary to use an adequate and appropriate model depending on the type of solar cells.

\subsection{Irradiance model}

The sunlight consists of not only direct irradiance but also diffuse and reflected irradiance. Therefore we need to know the irradiance considering all components. In this study, we use Erbs model [15]. The details of this model are described in Chapter 3. 


\subsection{Voltage drop model of diode}

The solar panels used in our experiments have diodes. Because the voltage is dropped at these diodes depending on the current, the voltage drop should be considered. We approximated this relationship between the current and voltage drop of the diode $V_{f}$ by a power function of the current $I$ given by:

$$
V_{f}=0.9826 I^{0.0981}
$$

\subsection{Solar constant and solar degradation effect}

The change of the solar constant and the degradation in the solar panels are considered as factors which affect the attitude determination system. The change of the solar constant is approximately $0.1 \%$ [16], and we found the effect to estimate values is less than 0.03 degrees by the numerical simulation. Thus we do not take it into account. In the other hands, radiation is much affects the characteristics of solar cells. In the UNISAT-3 mission the observed solar array degradation was very fast in the first half-year, during which approximately one-fourth of the initial efficiency was lost [14]. Although this type of degradation should be considered when the attitude is estimated using the current of the solar panels in actual space exploration missions, it is ignored here because the experiments were conducted on the earth. 


\section{Algorithm of IRAD}

IRAD focuses on the characteristics of the solar panels. Fig. 3 shows the conceptual diagram of IRAD. This method employs the current, voltage and temperature of the solar panels to estimate the sun vector, employs data from an accelerometer to estimate the gravity vector, and then combines the two vectors to estimate the attitude of a rover using the $q$ method [7]. In this study, we assumed the position of the rover is known, and that it does not move during attitude determination.

The direction of the sun is represented by the altitude angle $\theta_{s}$ and azimuth angle $\alpha_{s}$ as shown in Fig. 4. The sun vector $\mathbf{S}_{r}$ in the rover frame is represented by a vector as given by Eq. (2), which contains $\theta_{s}$ and $\alpha_{s}$. This equation implies that estimating $\mathbf{S}_{r}$ is the same as estimating $\theta_{s}$ and $\alpha_{s}$.

$$
\mathbf{S}_{r}=\left[\begin{array}{lll}
\cos \theta_{s} \cos \alpha_{s} & \cos \theta_{s} \sin \alpha_{s} & \sin \theta_{s}
\end{array}\right]^{T}
$$

As the current of solar panels are almost proportional to the irradiance, we derive a cost function using this characteristic to estimate the sun vector. Although we need the solar irradiance on inclined surface, generally we can only obtain the global solar irradiance. To obtain the irradiance on a tilted solar panel from measurements of global solar irradiance, the direct and diffuse components of global solar irradiance are required. Because the experiments in this study were carried out on the earth, we used the Erbs model [15] to estimate the components. Although the irradiance models are different, depending on the environment of the planet being explored, for example 
on the moon there is no diffuse solar radiation because there is no atmosphere, the model is easily created.

The global solar irradiance $H$ is represented as sum of the diffuse solar radiation and the direct solar radiation.

$$
H=H_{d}+H_{b},
$$

where $H_{d}$ and $H_{b}$ are the diffuse and direct solar radiation, respectively. The ratio of $H_{d}$ to $H_{b}$ is derived from:

$$
\text { if } \begin{aligned}
H / H_{0} & \leq 0.22 \\
& \frac{H_{d}}{H}=1.0-0.09 \frac{H}{H_{0}}
\end{aligned}
$$

if $0.22<H / H_{0} \leq 0.80$

$$
\frac{H_{d}}{H}=0.9511-0.1604 \frac{H}{H_{0}}+4.388\left(\frac{H}{H_{0}}\right)^{2}-16.638\left(\frac{H}{H_{0}}\right)^{3}+12.366\left(\frac{H}{H_{0}}\right)^{4}
$$

$$
\text { if } \begin{aligned}
0.80<H / H_{0} \\
\frac{H_{d}}{H}=0.165
\end{aligned}
$$

where $H_{0}$ is extraterrestrial solar radiation. The direct, reflected, and diffuse irradiance on inclined surface of each solar panel are given by:

$$
\begin{aligned}
& h_{b i}=H_{b} \frac{\cos \theta_{i}}{\cos \theta_{z}}, \\
& h_{r i}=H p \frac{1-\cos \theta_{a}}{2}, \\
& h_{d i}=H_{d} \frac{1+\cos \theta_{a}}{2},
\end{aligned}
$$


where $\theta_{z}$ is zenith angle, $\theta_{a}$ is inclination angle of the solar panel, $p$ is albedo, and $\theta_{i}$ is an angle of incidence of each solar panel. $\theta_{i}$ is derived from the definition of the inner product given by:

$$
\theta_{i}=\cos ^{-1}\left(\mathbf{n}_{i} \cdot \mathbf{S}_{r}\right)
$$

where $\mathbf{n}_{i}$ represents the normal vector of each solar panel. Consequently solar irradiance on the solar panel is given by:

$$
h_{i}=h_{b i}+h_{r i}+h_{d i} .
$$

We derive the cost function to estimate the sun vector using Eq. (11):

$$
J=\sum_{i=1}^{m}\left(\frac{h_{i}}{h_{0}}-\frac{I_{i}}{I_{i \max }}\right)^{2},
$$

where $m$ is the number of the light receiving solar panels and $h_{0}$ is the irradiance when $\theta_{i}=0 . I_{i \max }$ is the output current when the incident angle of the sunlight is normal to the surface of solar panels, and $I_{i}$ is the current of each solar panel obtained using current sensors. This cost function $J$ includes the sun vector $\mathbf{S}_{r}$, which includes the two variables, the sun altitude $\theta_{s}$ and sun azimuth $\alpha_{s}$. Because current of solar panels are almost proportional to the irradiance, the optimum sun altitude and azimuth are estimated as a pair which minimizes $J$. Because the variables included in $J$ are $\theta_{s}$ and $\alpha_{s}$, it is possible to solve this problem as a minimization problem of two variables. We use the Nelder-Mead Simplex method [17] to minimize the cost function. The initial guesses for $\theta_{s}$ and $\alpha_{s}$ are 45 degrees and 180 degrees respectively. The sun vector $\mathbf{S}_{r}$ is then estimated by substituting $\theta_{s}$ and $\alpha_{s}$, obtained using the minimization of $J$, into Eq. (2). 
In addition, the gravity vector for the rover frame can be obtained using the accelerometer data. The relationship to the rover frame is represented as follows:

$$
\mathbf{G}_{r}=\mathbf{A} \mathbf{G}_{t}+\mathbf{v}_{g},
$$

where $\mathbf{G}_{r}$ is the gravity vector in the rover frame, $\mathbf{G}_{t}$ is the gravity vector in the topocentric frame, $\mathbf{A}$ is the direction cosine matrix, and $\mathbf{v}_{g}$ is the sensor noise.

Thus we can estimate the sun vector and gravity vector in the rover frame. With these vectors, the attitude of the rover is estimated using the $q$ method [7], which uses the vectors in the reference frame and the measured or estimated vectors, and also obtains the direction cosine matrix $\mathbf{A}(\tilde{\mathbf{q}})$ described by the quaternion $\tilde{\mathbf{q}}$ using minimization of the loss function $L(\mathbf{A})$ given by:

$$
L(\mathbf{A})=\frac{1}{2} \sum_{i=1}^{n} a_{i}\left|\hat{\mathbf{W}}_{i}-\mathbf{A} \hat{\mathbf{V}}_{i}\right|^{2}
$$

where $a_{i}$ are the corresponding weights, $n$ is the number of vectors (sun vector and gravity vector, so $n=2$ ), $\hat{\mathbf{W}}_{i}$ are the observation unit vectors which are measured in the rover frame. In this study these vectors define the direction of the sun and gravity obtained using the current from the solar panels, and an accelerometer, respectively. $\hat{\mathbf{V}}_{i}$ are the reference unit vectors in the reference frame in which we want to estimate attitude, which is the topocentric frame in Fig. 1a, and the sun vector in an arbitrary position and time can be obtained from ephemeris [18]. We assume the reference unit vector of gravity is along $-Z_{t} . a_{i}$ is determined from the observation-error variances of the sun vector $\sigma_{1}^{2}$ and the gravity vector $\sigma_{2}^{2}$ :

$$
a_{1}=\frac{\sigma_{1}^{-2}}{\sum_{i=1}^{2} \sigma_{i}^{-2}}
$$




$$
a_{2}=\frac{\sigma_{2}^{-2}}{\sum_{i=1}^{2} \sigma_{i}^{-2}}
$$

We define $\sigma_{1}^{2}=3.24, \sigma_{2}^{2}=9.81 \times 10^{-5}$. These parameters were determined by experiment.

Using the shorthand $\mathrm{c} \theta=\cos \theta$, and $\operatorname{s} \theta=\sin \theta, \mathbf{A}(\tilde{\mathbf{q}})$ can be converted into Euler angle formulation given by:

$$
\mathbf{A}(\tilde{\mathbf{q}})=\left[\begin{array}{ccc}
\mathrm{c} \theta_{r} \mathrm{c} \psi_{r} & \mathrm{c} \theta_{r} \mathrm{~s} \psi_{r} & -\mathrm{s} \theta_{r} \\
\mathrm{~s} \phi_{r} \mathrm{~s} \theta_{r} \mathrm{c} \psi_{r}-\mathrm{c} \phi_{r} \mathrm{~s} \psi_{r} & \mathrm{~s} \phi_{r} \mathrm{~s} \theta_{r} \mathrm{~s} \psi_{r}+\mathrm{c} \phi_{r} \mathrm{c} \psi_{r} & \mathrm{~s} \phi_{r} \mathrm{c} \theta_{r} \\
\mathrm{c} \phi_{r} \mathrm{~s} \theta_{r} \mathrm{c} \psi_{r}+\mathrm{s} \phi_{r} \mathrm{~s} \psi_{r} & \mathrm{c} \phi_{r} \mathrm{~s} \theta_{r} \mathrm{~s} \psi_{r}-\mathrm{s} \phi_{r} \mathrm{c} \psi_{r} & \mathrm{c} \phi_{r} \mathrm{c} \theta_{r}
\end{array}\right]
$$

where $\phi_{r}, \theta_{r}$, and $\psi_{r}$ denote the roll, pitch and yaw angles respectively, described as:

$$
\begin{aligned}
& \phi_{r}=\tan ^{-1}\left(\frac{A_{2,3}}{A_{3,3}}\right), \\
& \theta_{r}=-\sin ^{-1} A_{1,3}, \\
& \psi_{r}=\tan ^{-1}\left(\frac{A_{1,2}}{A_{1,1}}\right) .
\end{aligned}
$$




\section{Preliminary analysis}

Because we use the data of the solar panels employed on a rover in IRAD, the accuracy of attitude determination may change depending on the arrangement of the solar panels and the position of the sun. Accordingly, as a preliminary analysis, we examine the necessary conditions for the arrangement of the solar panels and the position of the sun for applying IRAD. First, we examine the relationship between an angle of the solar panels employed on a rover and the accuracy of attitude determination. Next, we examine the relationship between the altitude of the sun and the accuracy of attitude determination. In this study, we assume there is no individual difference or degradation in the solar panels. Table 1 shows the parameters of the solar panel used in this study. Table 2 shows the parameters used in the numerical simulations.

\subsection{Arrangement of the solar panel}

In this section, we examine the relationship between the angle of the solar panels $\beta_{i}$ and the accuracy of attitude determination. In this study, we define an arrangement of solar panels with two parameters: the number of solar panels and the angle of the solar panels $\beta_{i}$. Fig. 5 shows an example of a pair of two parameters. In the numerical simulations, we examine the accuracy of attitude determination when the number of solar panels is five, seven and nine including one panel on the top, and $\beta_{i}$ is $0 \sim 90$ degrees in units of a degree. We assume the rover estimates its attitude every second. 
Fig. 6a-c show the roll, pitch, and yaw standard deviations of the estimate values for $180 \mathrm{~s}$. We see from Fig. 6a and $6 \mathrm{~b}$ that the estimated roll and pitch angle do not correlate with $\beta_{i}$. This is because the accuracy of roll and pitch depends on the estimate values of the gravity vector obtained using an accelerometer. In contrast, Fig. $6 \mathrm{c}$ shows the standard deviation of the yaw estimate value correlates with $\beta_{i}$, especially when $\beta_{i}$ is near 0 degree, that is, all of the solar panels are near horizontal. This is because the currents of all the solar panels become almost equal when they are near horizontal, and the noise of the current sensor then deteriorates the accuracy of attitude determination. Besides, as shown in Fig. 6c, a small but significant standard deviation appears when $\beta_{i}$ is near 90 degrees. The standard deviation is smaller when the solar panels increase in number, because the number of solar panels receiving sunlight decreases when they become near vertical, and the data for attitude determination then decreases. From these results, it is necessary to tilt the solar panels more than approximately 20 degrees to avoid deterioration in accuracy.

\subsection{Altitude of the sun}

In this section, we examine the impact on the accuracy of attitude determination when $\beta_{i}$ is fixed and the position of the sun changes. In the numerical simulations we examine the accuracy of attitude determination when $\beta_{i}$ is fixed at 45 degrees, except for the upper solar panel, and the altitude of the sun is $0 \sim 90$ degrees in units of a degree. The condition for the number of solar panels is the same as in the previous section. 
Fig. 7a-c shows the roll, pitch, and yaw standard deviations of the estimate values for $180 \mathrm{~s}$. We see from Fig. 7c that the standard deviation for the estimate value of the yaw is larger when the sun is nearly directly above the rover, because when in this position the difference in each solar panel current, depending on the change in yaw angle, becomes smaller, and the correlation correspondingly becomes smaller. This problem inevitably occurs when attitude is estimated using the sun. Furgale et al. [5] has examined estimation errors when an attitude determination system uses the sun. As shown in Fig. 7a-c, the standard deviation in all attitude angles is larger when the altitude of the sun is low, especially under 15 degrees. This is the same as in the previous section, when the available data for attitude determination decrease because the number of solar panels receiving sunlight decreases when the altitude of the sun becomes lower. From these results, it was found that the altitude of the sun should be between approximately $15 \sim 70$ degrees to avoid deterioration in accuracy. 


\section{Simulations}

In this chapter, numerical simulations of the determination of the rover attitude are conducted to show the effectiveness of IRAD. The four rover models used in numerical simulations are listed in Fig. 8. Rover Model 1 has one panel on top and eight panels on the side. Rover Model 2 has one panel on the top and four panels on the side. Rover Model 3 has four panels on the side. Rover Model 4 has eight panels on the side. We assume that the rover is on the Moon, and five sets of ephemeris data were used in the numerical simulations for the solar conditions shown in Table 3. According to the results shown in Chapter 4, we chose ephemeris data which were 15 $\sim 70$ degrees for the sun's altitude.

Deans et al. [19] stated that the attitude estimate error in the heading direction needs to reduce below 3 degrees to reduce position estimation error within $5 \%$ in the Mars rover missions. Therefore our goal was to reduce the estimation error to within 3 degrees.

Here rovers were assumed to estimate their attitude for $180 \mathrm{~s}$, and the sampling period of the sensors was $1 \mathrm{~s}$. All the initial attitude angles of the rovers were assumed to be 10 degrees. The most appropriate attitude estimate values at time $t$ are the mean values of all estimate values obtained before time $t$ because in this study we assumed the rovers do not move during attitude determination.

Table 4 shows attitude estimate values after attitude determination over $180 \mathrm{~s}$. As one example, Fig. 9 shows the numerical simulation results for the case with ephemeris 1.

We see from Fig. 9a-d that the estimate values converged in approximately $60 \mathrm{~s}$ for each rover model. However, we also see from Fig. 9b and Fig. 9c that the yaw estimate errors remained after estimate values converged. This is because few data 
can be used for attitude determination because the number of solar panels is smaller than for the other models as shown in Fig. 8b and Fig. 8c. In contrast, the estimates for the roll and pitch angles were better than for the yaw angle. This is because the gravity vector can be obtained more precisely than the sun vector, and estimate values of the roll and pitch angles were very dependent on the estimated gravity vector. As shown in the results, the estimate values for the roll, pitch and yaw angles converged within 3 degrees for all models. Furthermore, as shown in Table 4, the estimate values for the roll, pitch and yaw angles are within 3 degrees for all cases of ephemeris and rover models. 


\section{Attitude determination platform}

To validate IRAD, some experiments with solar panels and sensors were carried out. In this chapter, we describe the experimental setup and our means of determining the true values of the attitude, and experiment conditions.

\subsection{Experiment system}

The experiments were conducted at Keio University $\left(35^{\circ} 33.3^{\prime} \mathrm{N}\right.$ latitude and $139^{\circ} 39.2^{\prime} \mathrm{E}$ longitude). The experiments data were collected by experiment platform shown in Fig. 10. The platform includes solar panels, a pyranometer, an accelerometer, an electronic load, and a laptop computer to collect these data. Although this system was not an actuated rover, our focus with this platform is on problems of attitude determination, and thus it was sufficient as a means to collect data.

We measured the true north before the experiments were conducted to obtain the yaw true value of the attitude. Because magnetometer contains magnetic declination, we measured the direction of a shadow of a vertical rod at the time of culmination and we considered this direction as the true north. Therefore the yaw true value is the true north. The true values of roll and pitch angle were the average values of 18000 samples which obtained by an accelerometer. 


\subsection{Experiment conditions}

The experiments were conducted in the six conditions shown in Table 5. In each condition, voltage, current, and temperature of each solar panel, irradiance, and acceleration were logged at every 1 second. 


\section{Experimental results}

This chapter presents experimental results using IRAD. Fig. 11 shows current of each solar panel (SP) for Experiment 1. It was confirmed from Fig. 11 that the current of each solar panel is different depending on the angles of incidence for each solar panel. Table 6 shows the mean values and standard deviations of the current errors between the model described in Chapter 2 and measured values.

We applied IRAD to the current data and estimated the attitude. Fig. 12 shows the experiment results for Experiment 1. Although the estimate values for the roll and pitch angle have small errors due to high precision of the accelerometer to obtain the gravity vector, the estimate values of the yaw angle include relatively large noise. This is because the current sensors have noise shown in Fig. 11. As mentioned in Chapter 5, because we assumed the rovers do not move during attitude determination, the most appropriate attitude estimate values at time $t$ were obtained using a filter which derives the mean values of all estimate values obtained before time $t$. Fig. 13 shows the results using this filter. The estimate values for the roll, pitch and yaw angle converge in approximately $60 \mathrm{~s}$.

The estimate errors for all experiments are shown in Table 7. It was confirmed from Table 7 that the estimate errors for the yaw angle are larger than the roll and pitch angle. Although the estimate errors are small for Experiments 1, 2 and 4, they deteriorate for Experiment 3. This is because only two solar panels received sunlight. In Experiments 5 and 6, the estimate errors are relatively large. The cause is as follows: the panels are nearly horizontal in Experiments 5 and 6, and current of solar panels is barely depended on the direction of the sun. 


\section{Conclusions}

We have proposed an attitude determination system which we call Irradiancebased Attitude Determination (IRAD), as a redundant system that employs the characteristics of solar panels and an accelerometer in planetary exploration rovers. In IRAD, the solar panels' current, voltage, temperature and normal vectors were employed to estimate the sun vector, and an accelerometer was used to obtain the gravity vector. The attitude of a rover can be estimated using the $q$ method with the two vectors. Because IRAD does not need any external sensors such as cameras or sun sensors, but only internal sensors, it is easy to apply to rovers. Because we use the solar panels' normal vectors, IRAD can be applied to various shapes of rovers. The preliminary analysis shows the necessary conditions for the arrangement of the solar panels and the position of the sun for applying IRAD. The experiments results indicate that IRAD can estimate the roll, pitch, and yaw angles within a few degrees of accuracy.

Although the maximum current delivered by the solar panels is known and fixed in this study, it varies as a result of clouds or wind-blown dust, depending on the planetary environment. In this case the maximum current should be estimated to avoid degradation of the attitude estimation accuracy. This needs to be carried out in future work. In addition, it is assumed that the position of the rover is known in this study, and IRAD is required to be combined with the localization system in order to build the integrated navigation system. 


\section{References}

[1] J. K. Erickson, J. L. Callas, A. F. C. Haldemann, The Mars Exploration Rover Project: 2005 surface operations results, Acta Astronautica 61 (2007) 699-706.

[2] J. P. Grotzinger, J. Crisp, A. R. Vasavada, R. C. Anderson, C. J. Baker, R. Barry, D. F. Blake, P. Conrad, K. S. Edgett, B. Ferdowski, R. Gellert, J. B. Gilbert, M. Golombek, J. Gómez-Elvira, D. M. Hassler, L. Jandura, M. Litvak, P. Mahaffy, J. Maki, M. Meyer, M. C. Malin, I. Mitrofanov, J. J. Simmonds, D. Vaniman, R. V. Welch, R. C. Wiens, Mars Science Laboratory Mission and science investigation, Space Science Reviews 170 (2012) 5-56.

[3] T. Hashimoto, T. Hoshino, S. Tanaka, M. Otsuki, H. Otake, H. Morimoto, Japanese moon lander SELENE-2 Present status in 2009, Acta Astronautica 68 (2011) 1386-1391.

[4] K. S. Ali, C. A. Vanelli, J. J. Biesiadecki, M. W. Maimone, A. M. San Martin, J. W. Alexander, Attitude and Position Estimation on the Mars Exploration Rovers, 2005 IEEE International Conference on Systems, Man and Cybernetics 1 (2005) 20-27.

[5] P. Furgale, J. Enright, T. Barfoot, Sun Sensor Navigation for Planetary Rovers: Theory and Field Testing, IEEE Transactions on Aerospace and Electronic Systems 47 (3) (2011) 1631-1647.

[6] J. Amos, Mars rover: Wind sensor damaged on Nasa's Curiosity (August 21, 2012) , BBC NEWS, http://www.bbc.co.uk/news/science-environment-19338870. (as of July 2014)

[7] M. D. Shuster, S. D. Oh, Three-Axis Attitude Determination from Vector Observation, Journal of Guidance and Control 4 (1) (1981) 70-77. 
[8] F. Santoni, F. Bolotti, Attitude Determination of Small Spinning Spacecraft Using Three Axis Magnetometer and Solar Panels Data, IEEE Aerospace Conference Proceedings 7 (2000) 127-133.

[9] F. Santoni, F. Piergentili, UNISAT-3 attitude determination using solar panel and magnetometer data, The 56th International Astronautical Congress (2005).

[10]R. Burton, S. M. Rock, J. Springmann, J. Cutler, Online Attitude Determination of a Passively Magnetically Stabilized Spacecraft, The 23rd AAS/AIAA Space Flight Mechanics Meeting (2013).

[11]G. Walker, EVALUATING MPPT CONVERTER TOPOLOGIES USING A MATLAB PV MODEL, Journal of Electrical \& Electronics Engineering 21 (1) (2001) 49-56.

[12]M. G. Villalva, J. R. Gazoli, E. R.Filho, Modeling and circuit-based simulation of photovoltaic arrays, Brazilian Power Electronics Conference (2009) 1244-1254.

[13]K. Ishaque, Z. Salam, H. Taheri, Accurate MATLAB Simulink PV System Simulator Based on a Two-Diode Model, Journal of Power Electronics 11 (2) (2011) 179-187.

[14]F. Santoni, F. Piergentili, Analysis of the UNISAT-3 Solar Array In-Orbit Performance, Journal of Spacecraft and Rockets 45 (1) (2008) 142-148

[15]D. G. Erbs, S. A. Klein, J. A. Duffie, Estimation of the Diffuse Radiation Fraction for Hourly, Daily and Monthly Average Global Radiation, Solar Energy 28 (4) (1982) 293-302.

[16] R. C. Willson, H. S. Hudson, The Sun's luminosity over a complete solar cycle, Nature 351 (1991) 42-44. 
[17]J. C. Lagarias, J. A. Reeds, M. H. Wright, P. E. Wright, Convergence Properties of the Nelder-Mead Simplex Method in Low Dimensions, Society for Industrial and Applied Mathematics 9 (1) (1998) 112-147.

[18]HORIZONS System, http://ssd.jpl.nasa.gov/?horizons. (as of July 2014)

[19]M. C. Deans, D. Wettergreen, D. Villa, A sun tracker for planetary analog rovers, The 8th International Symposium on Artificial Intelligence, Robotics and Automation in Space (2005). 


\section{Vitae}

T. Ishida received the B.S. degree in engineering from department of system design engineering, Keio University, Yokohama, Japan, in 2013. His research interest includes motion control and attitude determination of automotive systems and robotics. He is a student member of The Japan Society of Mechanical Engineers.

M. Takahashi received the Ph.D. degrees in engineering in from the school of science for open and environmental systems, Keio University, in 2002 and 2004 respectively. From 2005 to 2008, he worked as a Research Assistant of the Department of System Design Engineering, Keio University, Yokohama, Japan and became an Associate Professor in 2009. His primary research interests include human-robot interaction, motion and vibration control, and sensor fusion. He is a member of American Institute of Aeronautics and Astronautics, The Japan Society of Mechanical Engineers and The Robotics Society of Japan. 


\section{Figure Captions}

Fig. 1.

(a) Topocentric frame which is defined with respect to the local horizon. (b) Rover frame in which the origin is at the rover.

Fig. 2.

Equivalent circuit of a cell of a solar panel described in the two-diode model [11].

Fig. 3.

Conceptual diagram of IRAD.

Fig. 4.

Sun vector in rover frame.

Fig. 5.

Example of the solar panels arrangement. Angle of solar panels is 30 degrees for eight solar panels on the side. 


\section{Fig. 6.}

Effect of the angle of solar panels on the attitude error standard deviation (five, seven, and nine solar panels). (a) roll, (b) pitch, and (c) yaw angle.

\section{Fig. 7.}

Effect of the sun altitude on the attitude error standard deviation (five, seven, and nine solar panels), (a) roll, (b) pitch, and (c) yaw angle.

Fig. 8.

Four rover models used in the numerical simulations, (a) Model 1, eight solar panels on the side and one on the top, (b) Model 2, four solar panels on the side and one on the top, (c) Model 3, four solar panels on the side, (d) Model 4, eight solar panels on the side.

\section{Fig. 9.}

Time history of the attitude estimate error (roll, pitch, and yaw), (a) - (d) show for Rover Models 1 to 4.

\section{Fig. 10.}

Experiment platform (shown here on Keio University). 
Fig. 11.

Output current of solar panels (Experiment 1, SP: Solar Panel).

Fig. 12.

Time history of the attitude estimate error (Experiment 1, raw).

Fig. 13.

Time history of the attitude estimate error (Experiment 1, filtered). 


\section{Tables}

Table 1

Parameters of the solar panel

\begin{tabular}{ccc}
\hline \hline Parameter & Unit & Value \\
\hline Short circuit current & $\mathrm{A}$ & 1.117 \\
Open circuit voltage & $\mathrm{V}$ & 10.470 \\
Short circuit current coefficient & $\%$ & +0.1 \\
Open circuit voltage coefficient & $\%$ & -0.38 \\
Cell number & - & 18 \\
Series resistance & $\Omega$ & 0.793 \\
Shunt resistance & $\Omega$ & 265.451 \\
Current at maximum power point & $\mathrm{A}$ & 1.026 \\
Voltage at maximum power point & $\mathrm{V}$ & 8.913 \\
\hline
\end{tabular}


Table 2

Parameters of numerical simulation

\begin{tabular}{ccc}
\hline \hline Parameter & Unit & Value \\
\hline Standard deviation (current) & $\mathrm{A}$ & 0.05 \\
Standard deviation (temperature) & $\mathrm{deg}$ & 1 \\
Standard deviation (gravity) & $\mathrm{m} / \mathrm{s}^{2}$ & 0.3 \\
Irradiance & $\mathrm{W} / \mathrm{m}^{2}$ & 1366 \\
Albedo & - & 0.1 \\
Temperature & $\mathrm{deg}$ & 25 \\
\hline
\end{tabular}


Table 3

Parameters of ephemeris data

\begin{tabular}{cccc}
\hline \hline Ephemeris & Longitude & Latitude & Sun altitude [deg] \\
\hline 1 & $15^{\circ} \mathrm{E}$ & $60^{\circ} \mathrm{N}$ & 21.4 \\
2 & $30^{\circ} \mathrm{E}$ & $30^{\circ} \mathrm{N}$ & 30.6 \\
3 & $0^{\circ} \mathrm{E}$ & $45^{\circ} \mathrm{N}$ & 43.5 \\
4 & $0^{\circ} \mathrm{E}$ & $30^{\circ} \mathrm{N}$ & 52.1 \\
5 & $0^{\circ} \mathrm{E}$ & $0^{\circ} \mathrm{N}$ & 66.3 \\
\hline
\end{tabular}


Table 4

Attitude determination error at $180 \mathrm{~s}$

\begin{tabular}{ccccccc}
\hline \hline \multicolumn{2}{c}{ Ephemeris } & 1 & 2 & 3 & 4 & 5 \\
\hline \multirow{2}{*}{ Rover } & Roll & 0.29 & 0.02 & 0.00 & 0.02 & 0.02 \\
Model 1 & Pitch & 0.19 & -0.01 & 0.01 & 0.01 & -0.01 \\
& Yaw & -0.64 & 0.23 & -0.02 & -0.05 & -1.23 \\
\hline \multirow{2}{*}{ Rover } & Roll & -0.02 & -0.10 & -0.08 & -0.19 & -0.36 \\
Model 2 & Pitch & 0.00 & -0.02 & -0.14 & -0.15 & 0.05 \\
& Yaw & -1.89 & -0.14 & -0.12 & -2.17 & 0.53 \\
\hline \multirow{2}{*}{ Rover } & Roll & -0.15 & -0.26 & -0.08 & -0.03 & -0.21 \\
Model 3 & Pitch & -0.08 & -0.02 & -0.17 & -0.03 & 0.01 \\
& Yaw & 1.26 & 2.87 & 2.15 & -0.60 & 0.76 \\
\hline \multirow{2}{*}{ Rover } & Roll & -0.24 & 0.22 & 0.12 & 0.43 & 0.25 \\
Model 4 & Pitch & -0.15 & 0.07 & 0.29 & 0.35 & -0.03 \\
& Yaw & -0.25 & -0.30 & 0.63 & -0.24 & -0.98 \\
\hline
\end{tabular}




\section{Table 5}

Experiment conditions of solar panels

\begin{tabular}{ccc}
\hline \hline $\begin{array}{c}\text { Experiment } \\
\text { No. }\end{array}$ & $\begin{array}{c}\text { Number of } \\
\text { solar panels }\end{array}$ & $\begin{array}{c}\text { Angle of } \\
\text { solar panels [deg] }\end{array}$ \\
\hline 1 & 4 & 45 \\
2 & 8 & 45 \\
3 & 4 & 60 \\
4 & 8 & 60 \\
5 & 4 & 30 \\
6 & 8 & 30 \\
\hline
\end{tabular}


Table 6

Current error between model and measured value

\begin{tabular}{ccc}
\hline \hline Solar panel & $\begin{array}{c}\text { Mean of } \\
\text { current error [A] }\end{array}$ & $\begin{array}{c}\text { Standard deviation of } \\
\text { current error [A] }\end{array}$ \\
\hline 1 & 0.083 & 0.038 \\
2 & 0.046 & 0.038 \\
3 & 0.024 & 0.045 \\
4 & -0.013 & 0.038 \\
\hline
\end{tabular}


Table 7

Attitude estimate error at $180 \mathrm{~s}$

\begin{tabular}{cccc}
\hline \hline $\begin{array}{c}\text { Experiment } \\
\text { No. }\end{array}$ & $\begin{array}{c}\text { Roll } \\
\text { Error [deg] }\end{array}$ & $\begin{array}{c}\text { Pitch } \\
\text { Error [deg] }\end{array}$ & $\begin{array}{c}\text { Yaw } \\
\text { Error [deg] }\end{array}$ \\
\hline 1 & 0.07 & -0.03 & -0.82 \\
2 & 0.02 & 0.01 & -1.21 \\
3 & -0.03 & 0.07 & -4.97 \\
4 & -0.03 & 0.06 & -1.89 \\
5 & -0.03 & 0.04 & -4.56 \\
6 & 0.02 & 0.02 & -3.06 \\
\hline
\end{tabular}




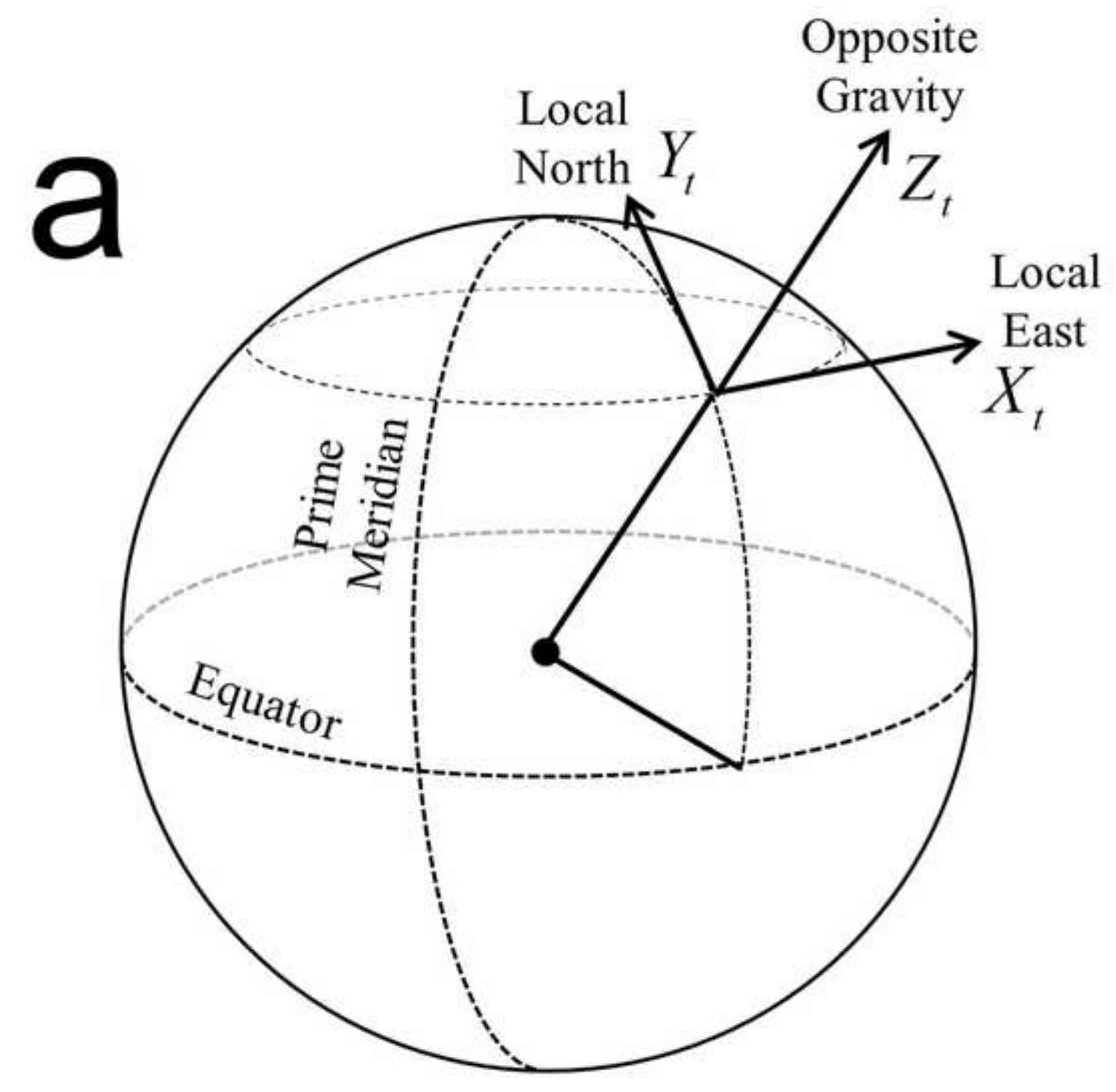

Opposite 


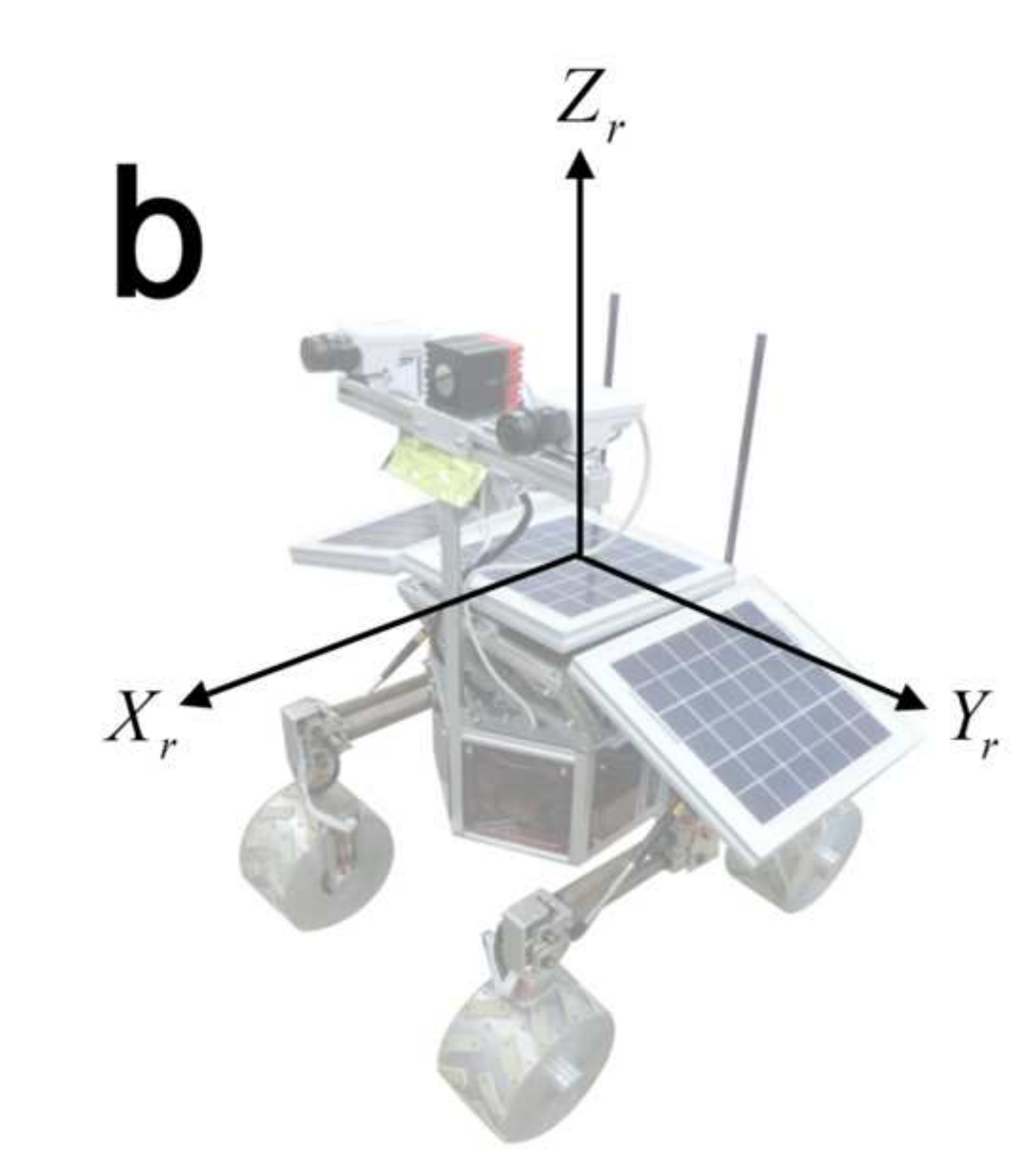

Figure1b (web)

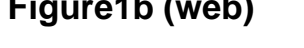

$+$

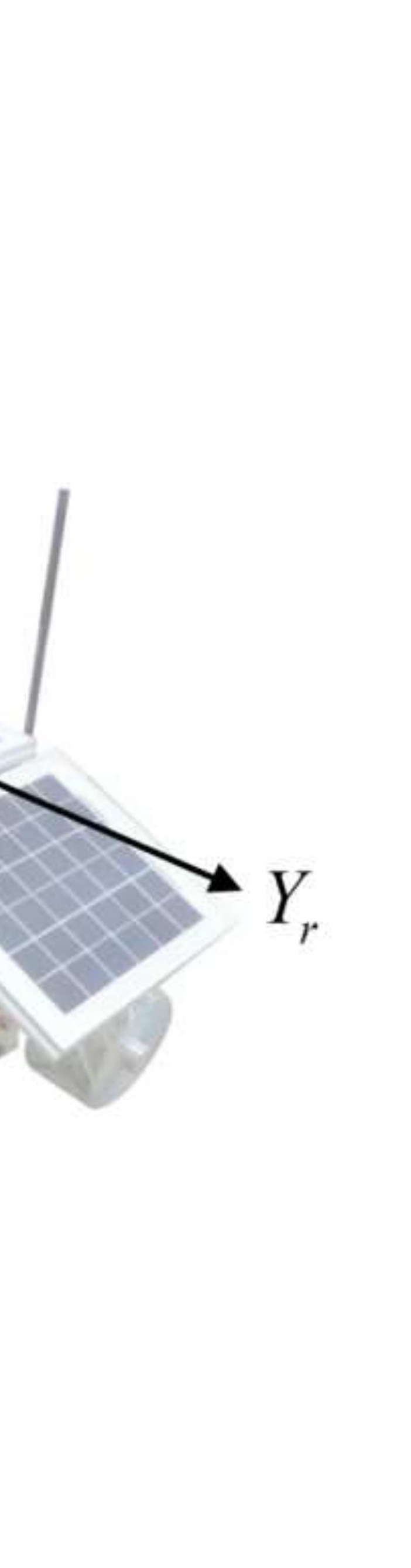




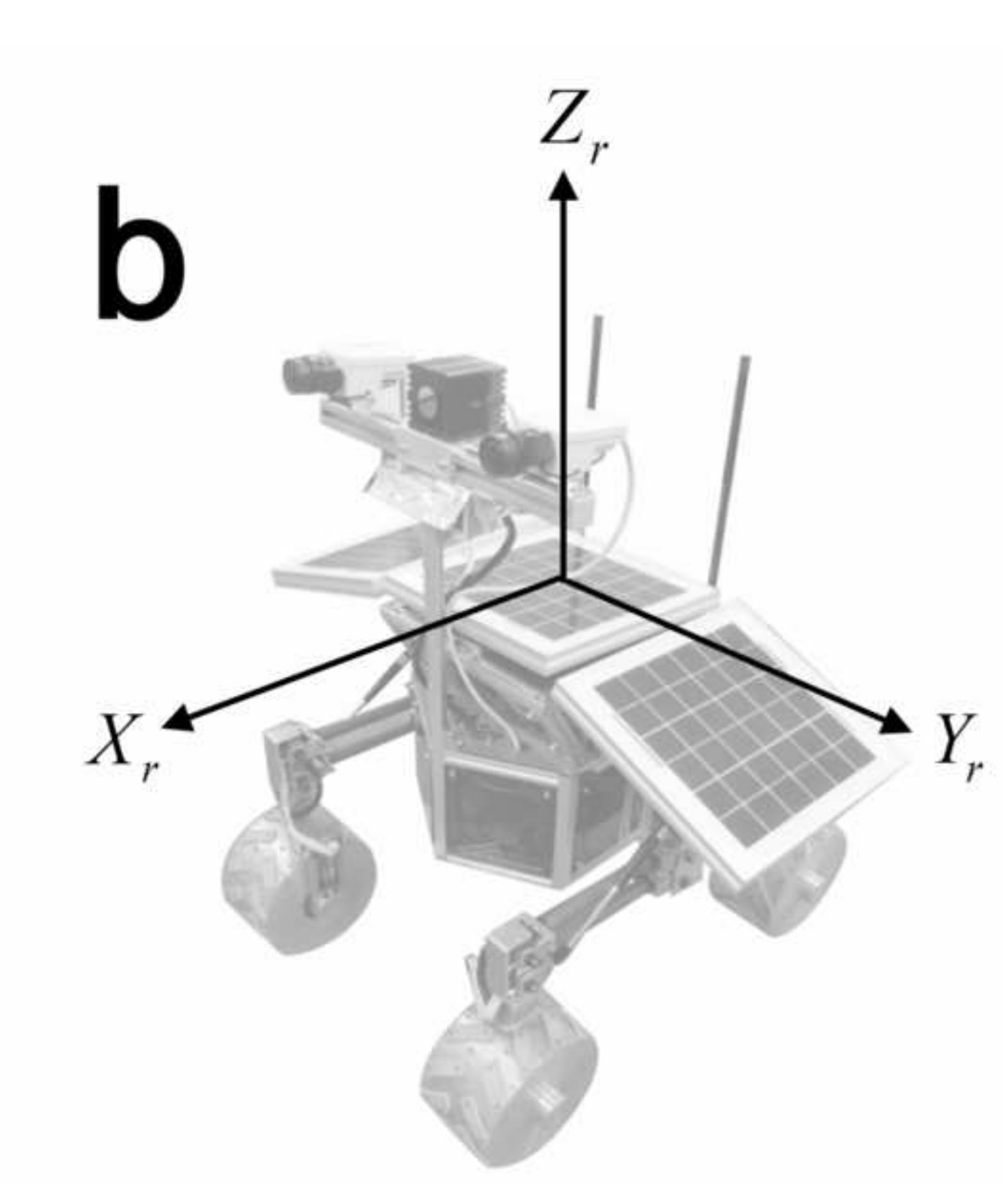

Figure1b (print)

(1)

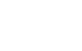

-
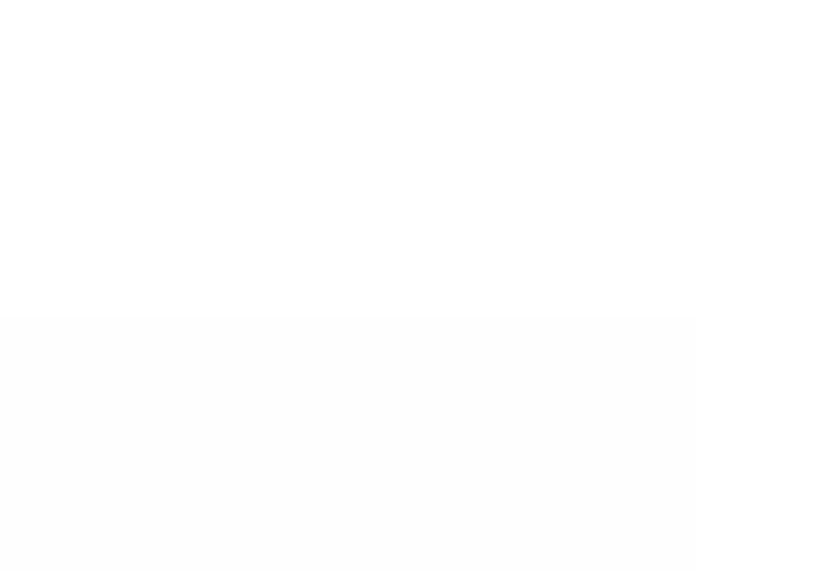


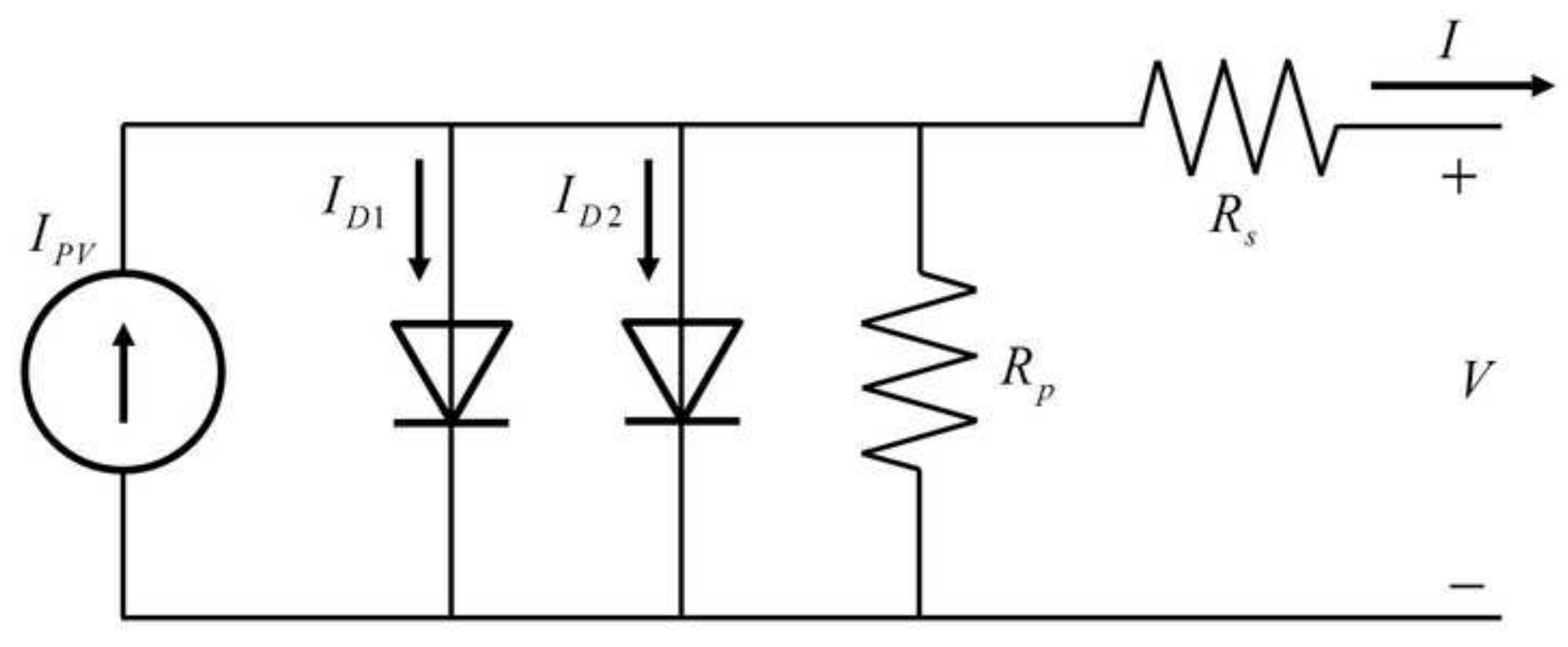




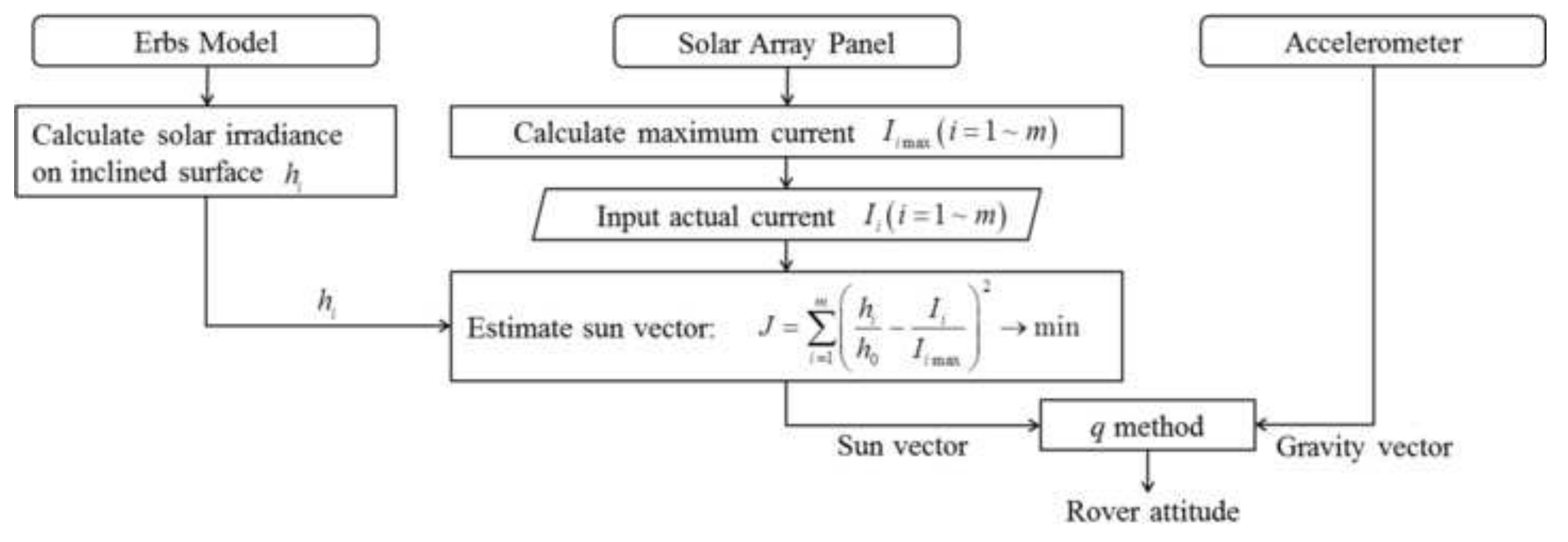




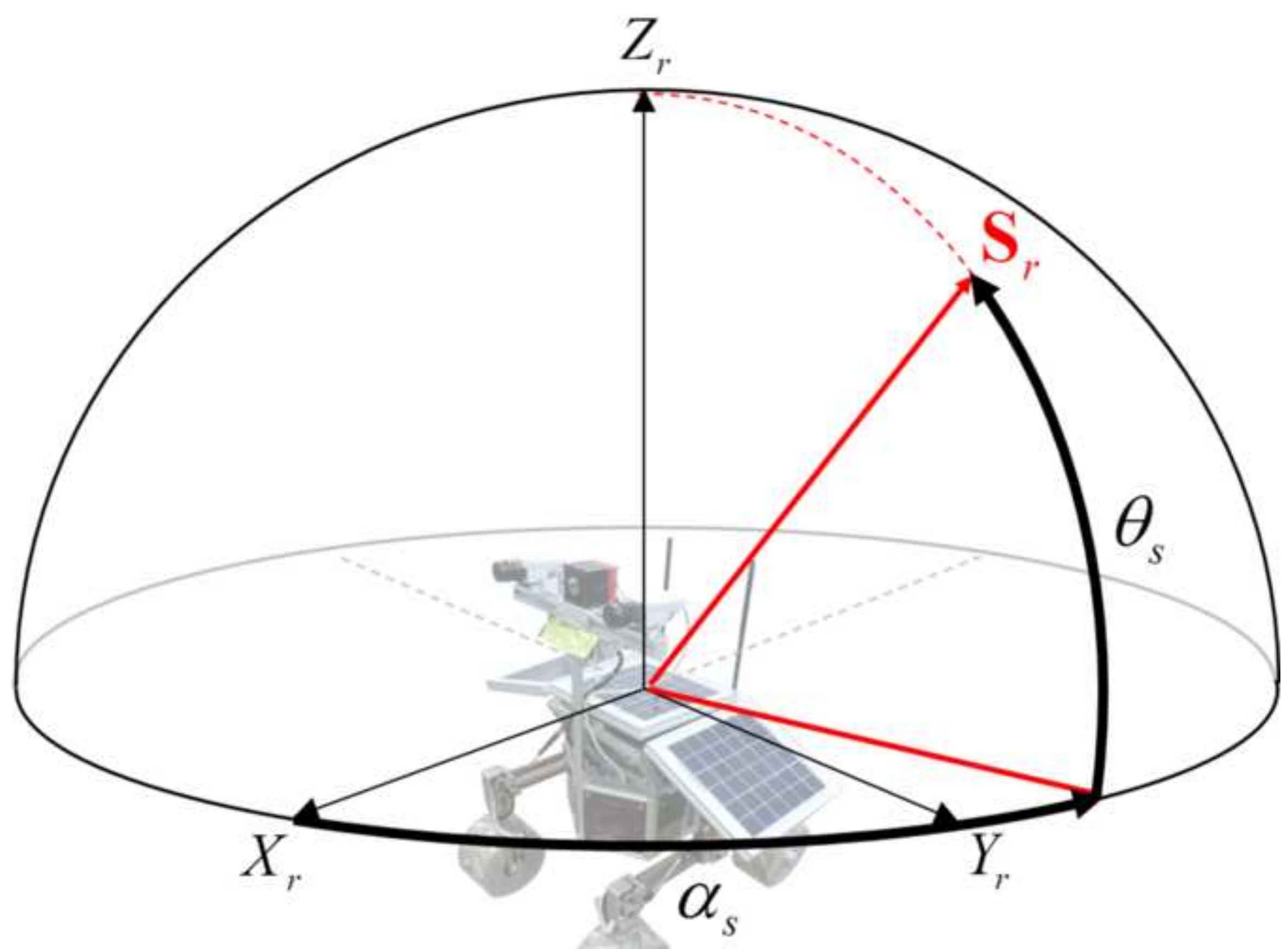




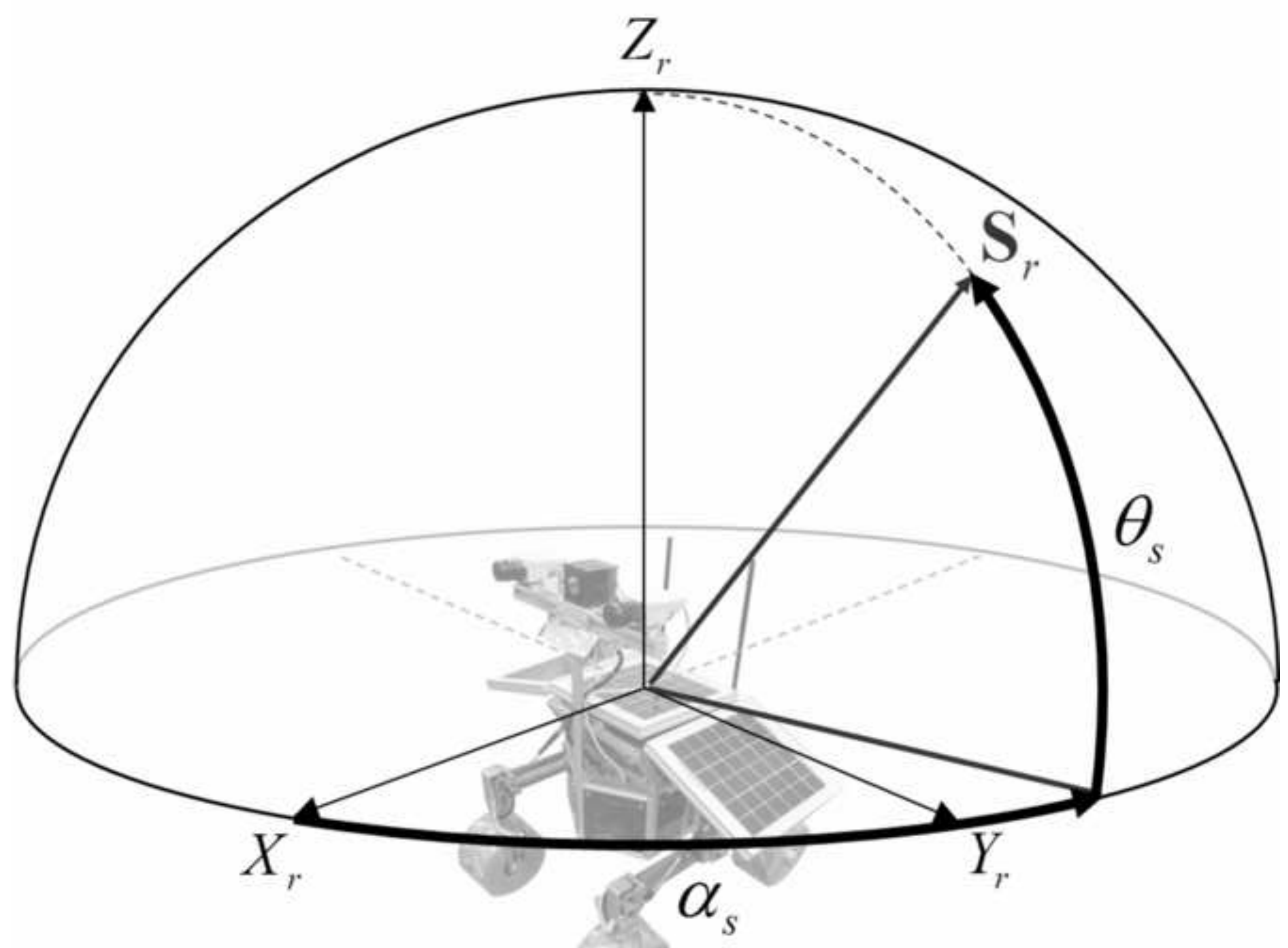


Figure5 (web)

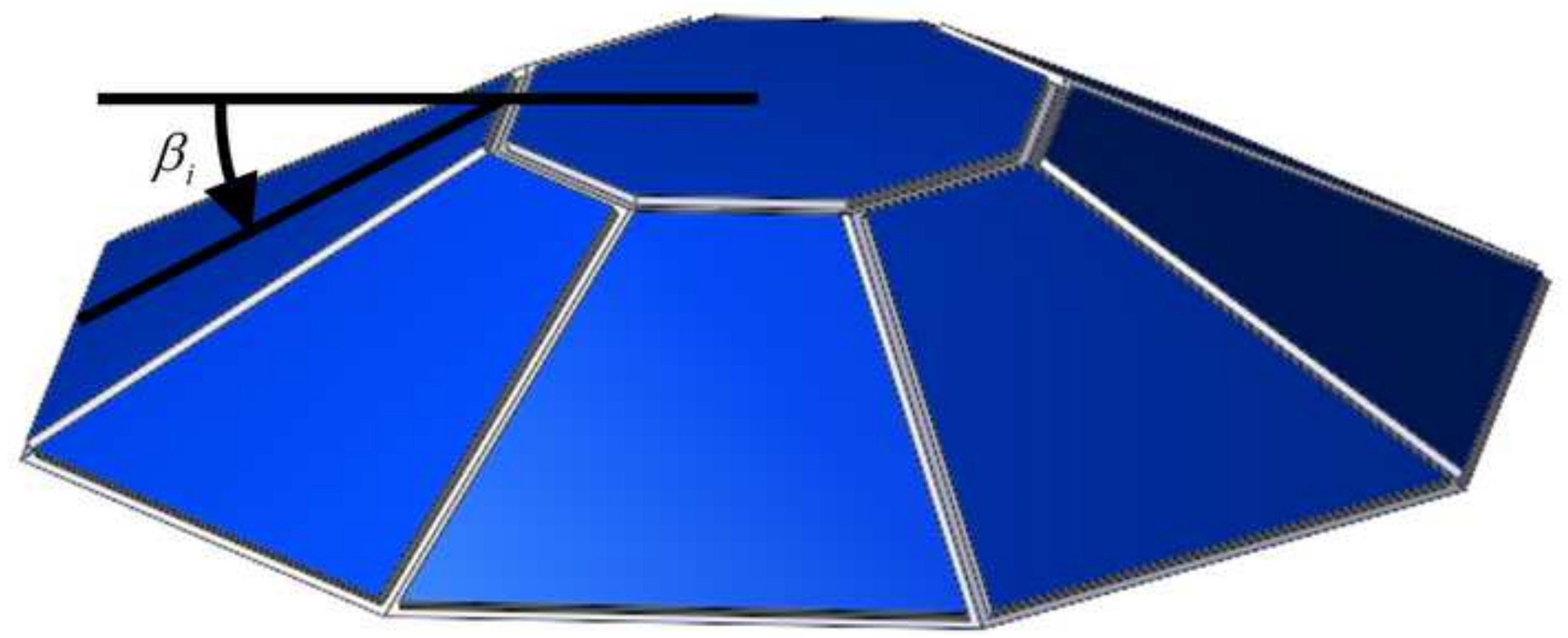




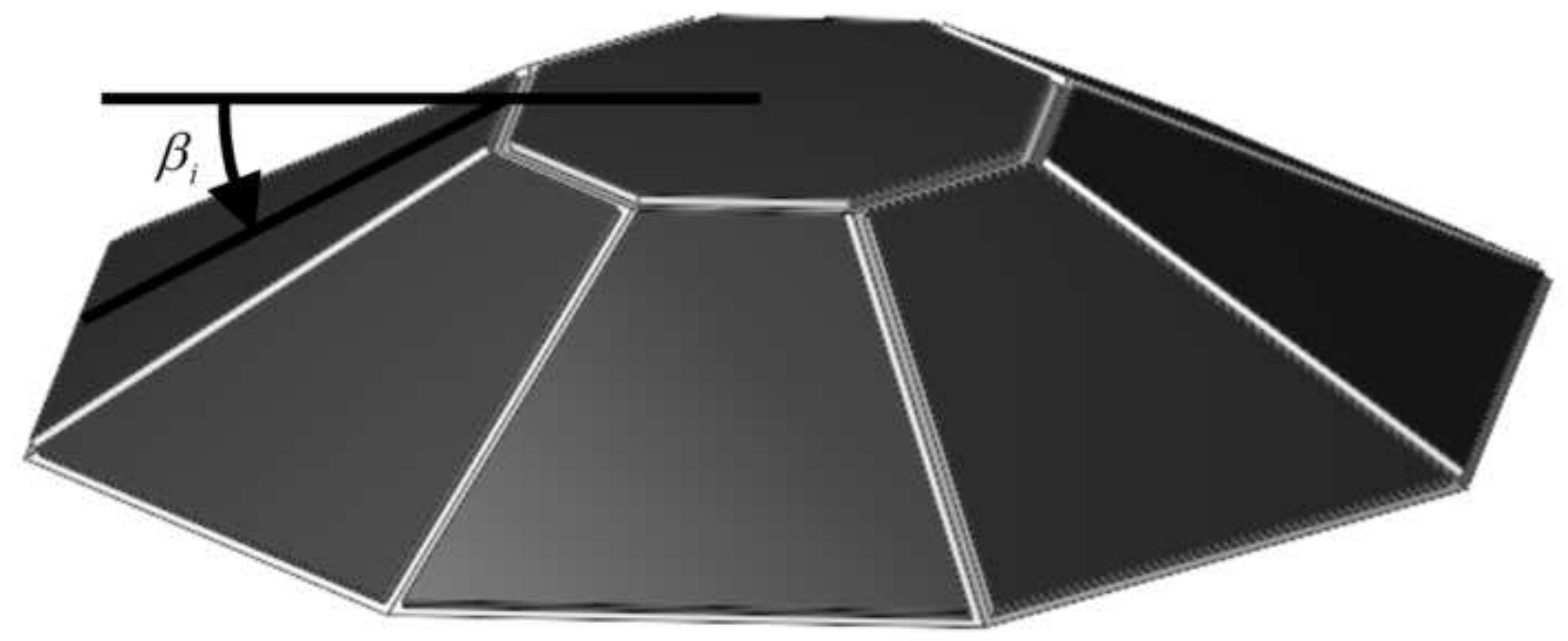

\section{Figures (print)}




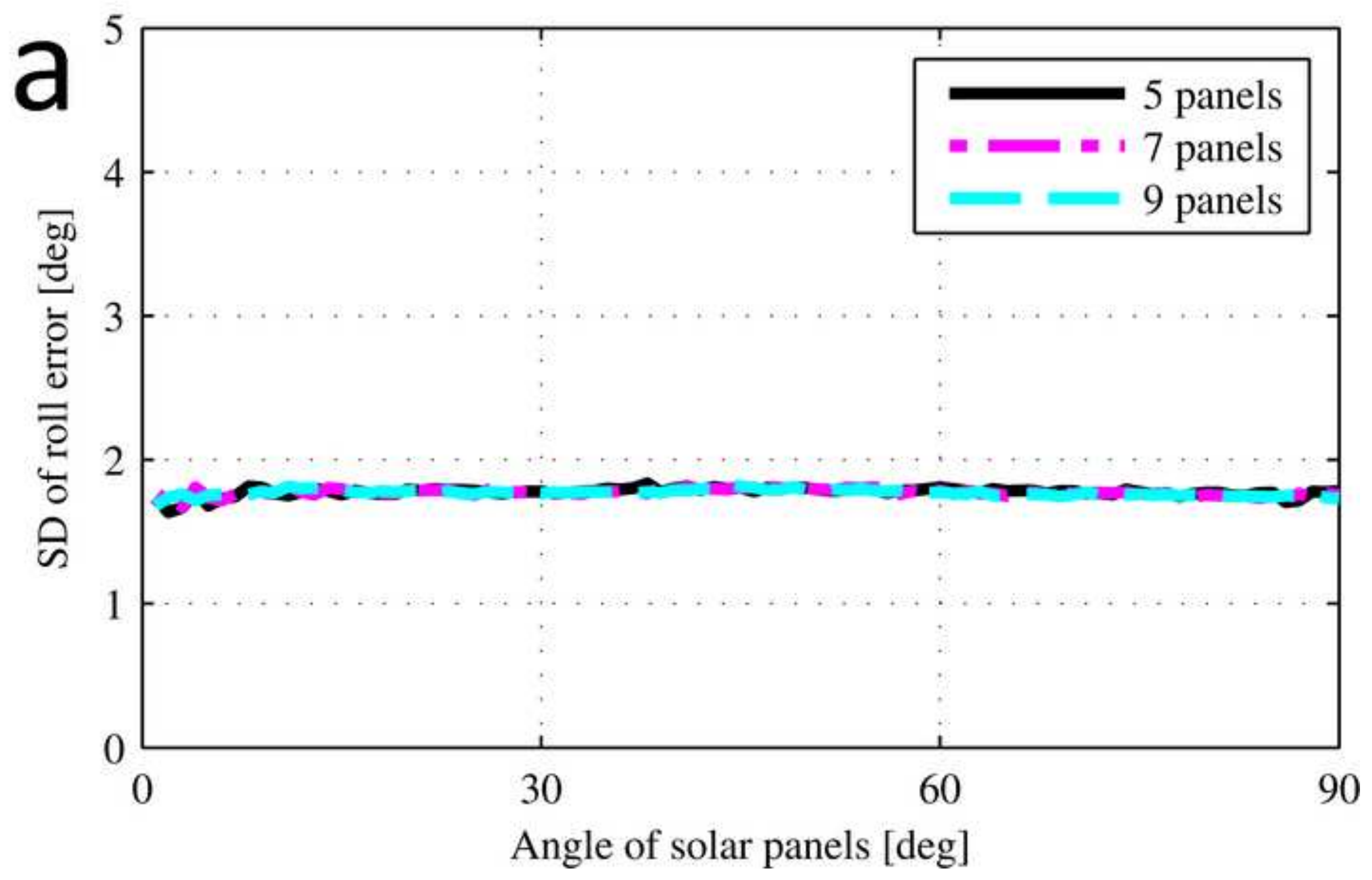




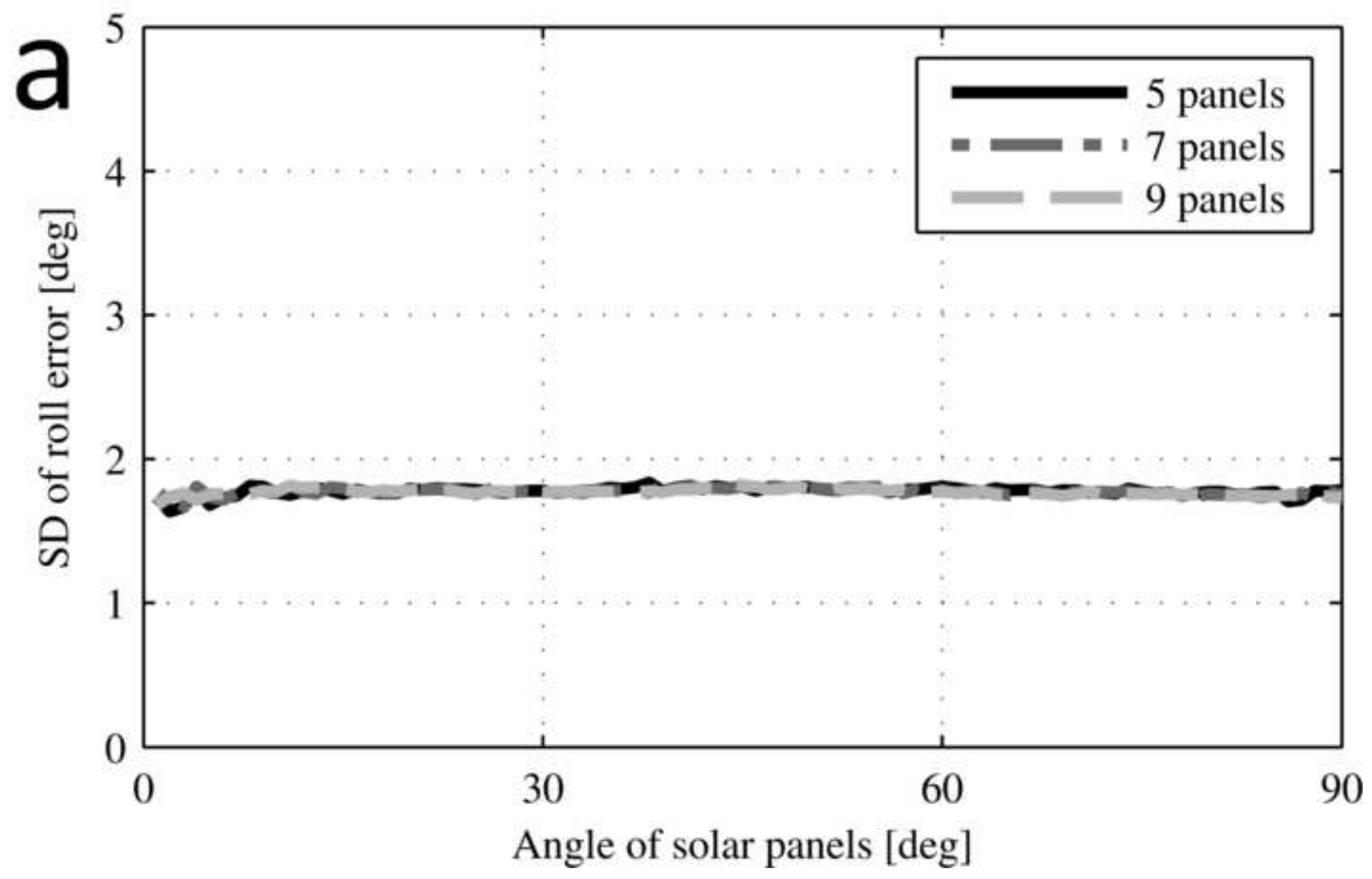




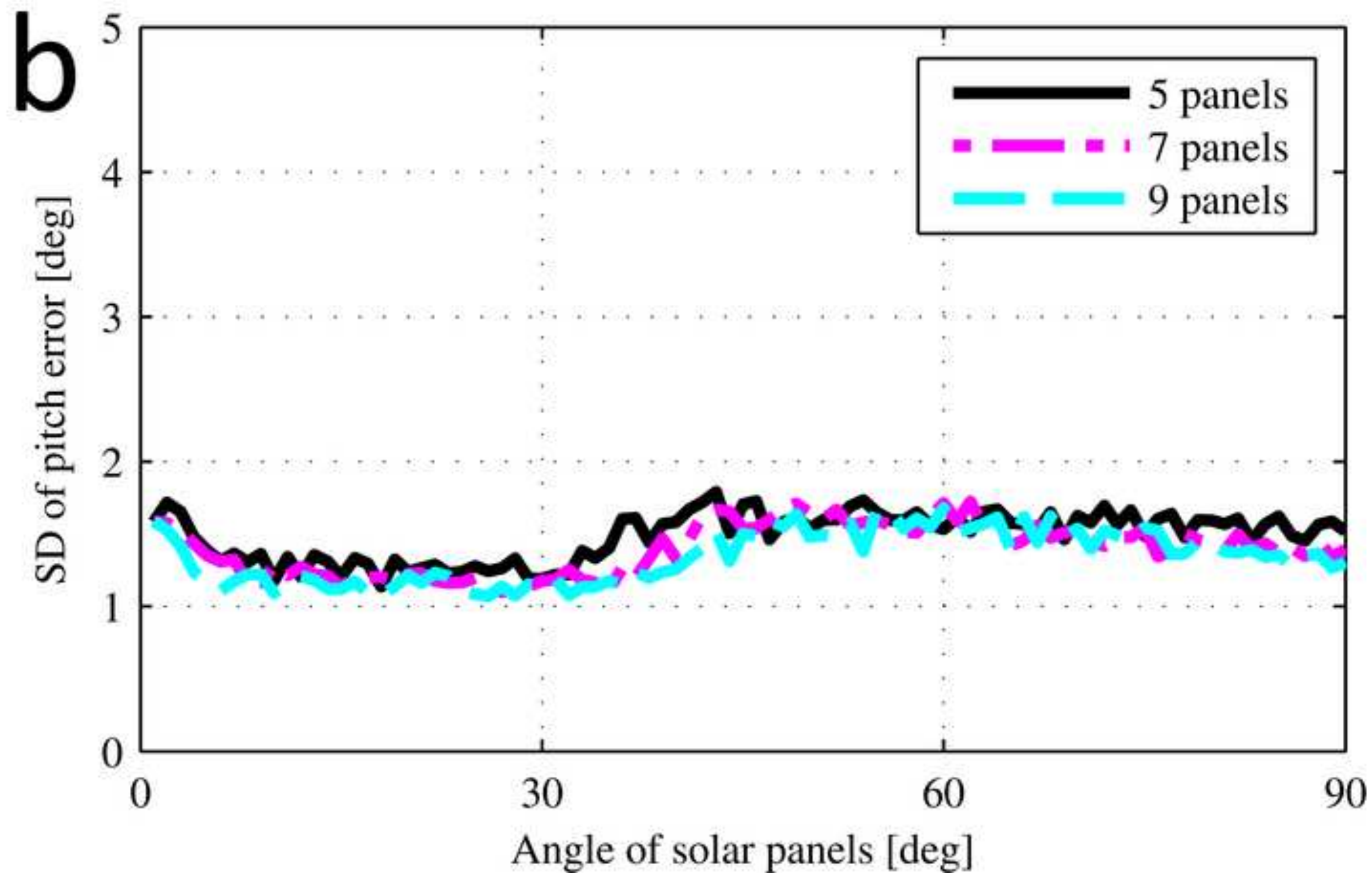




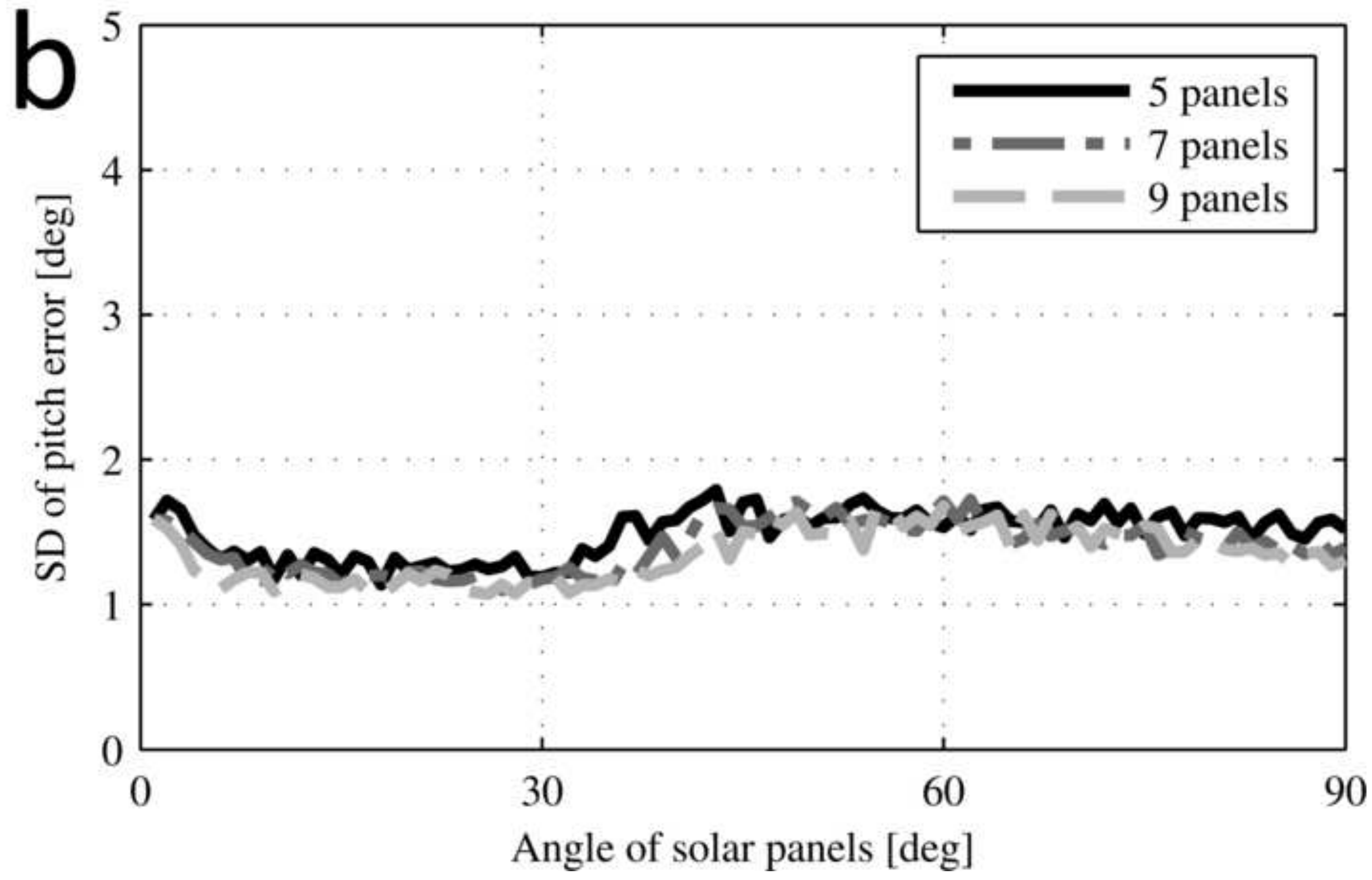




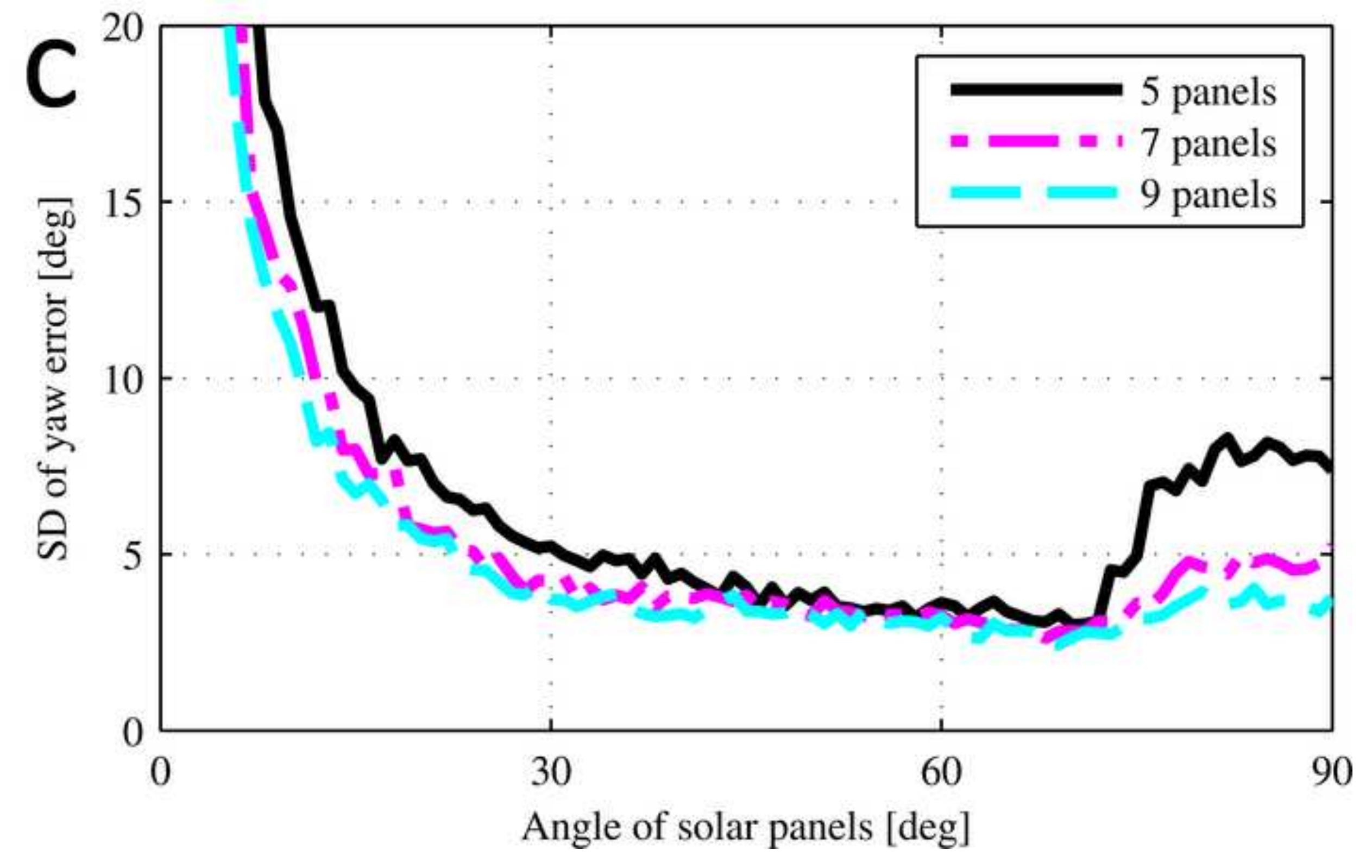




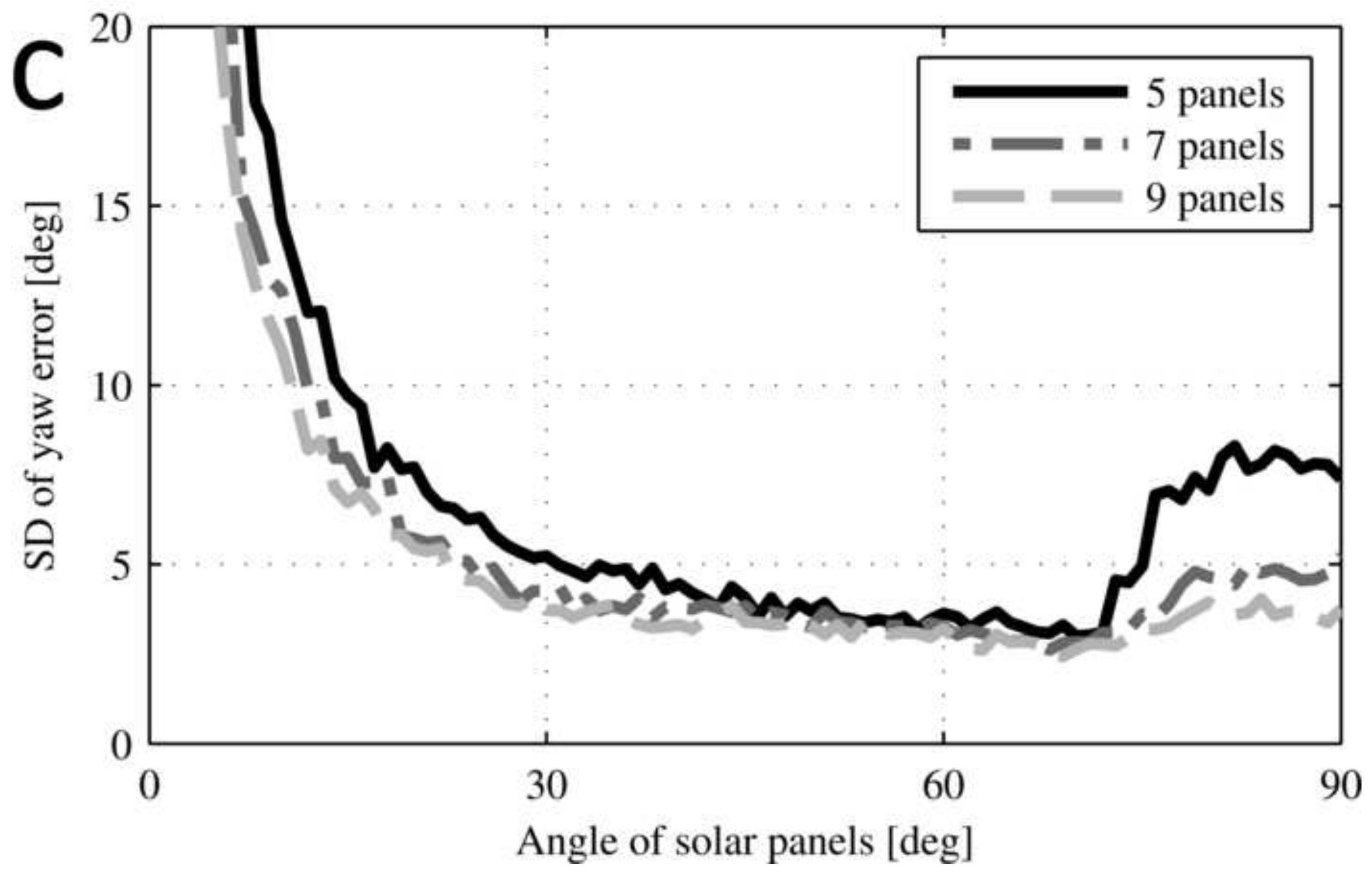




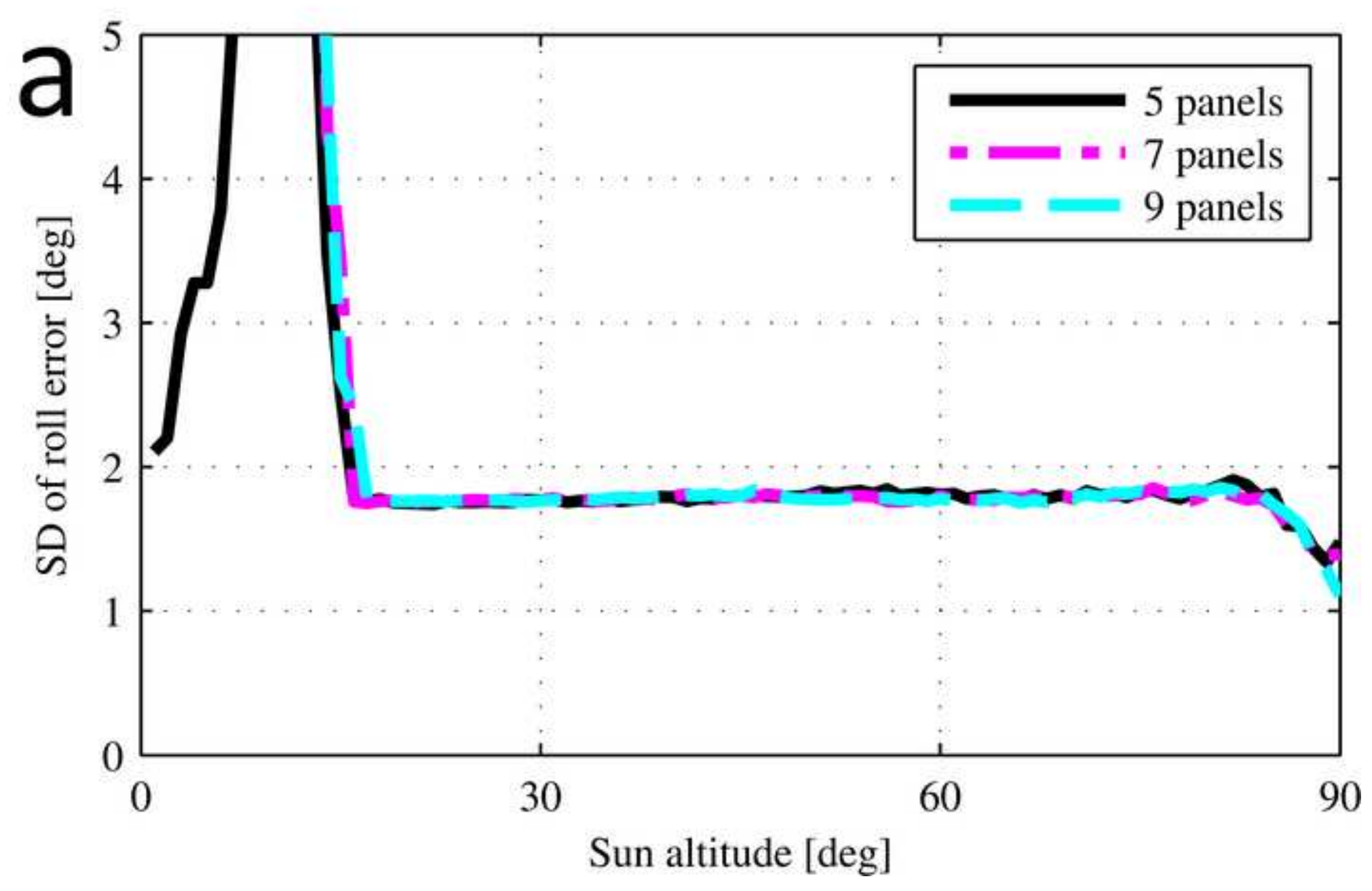




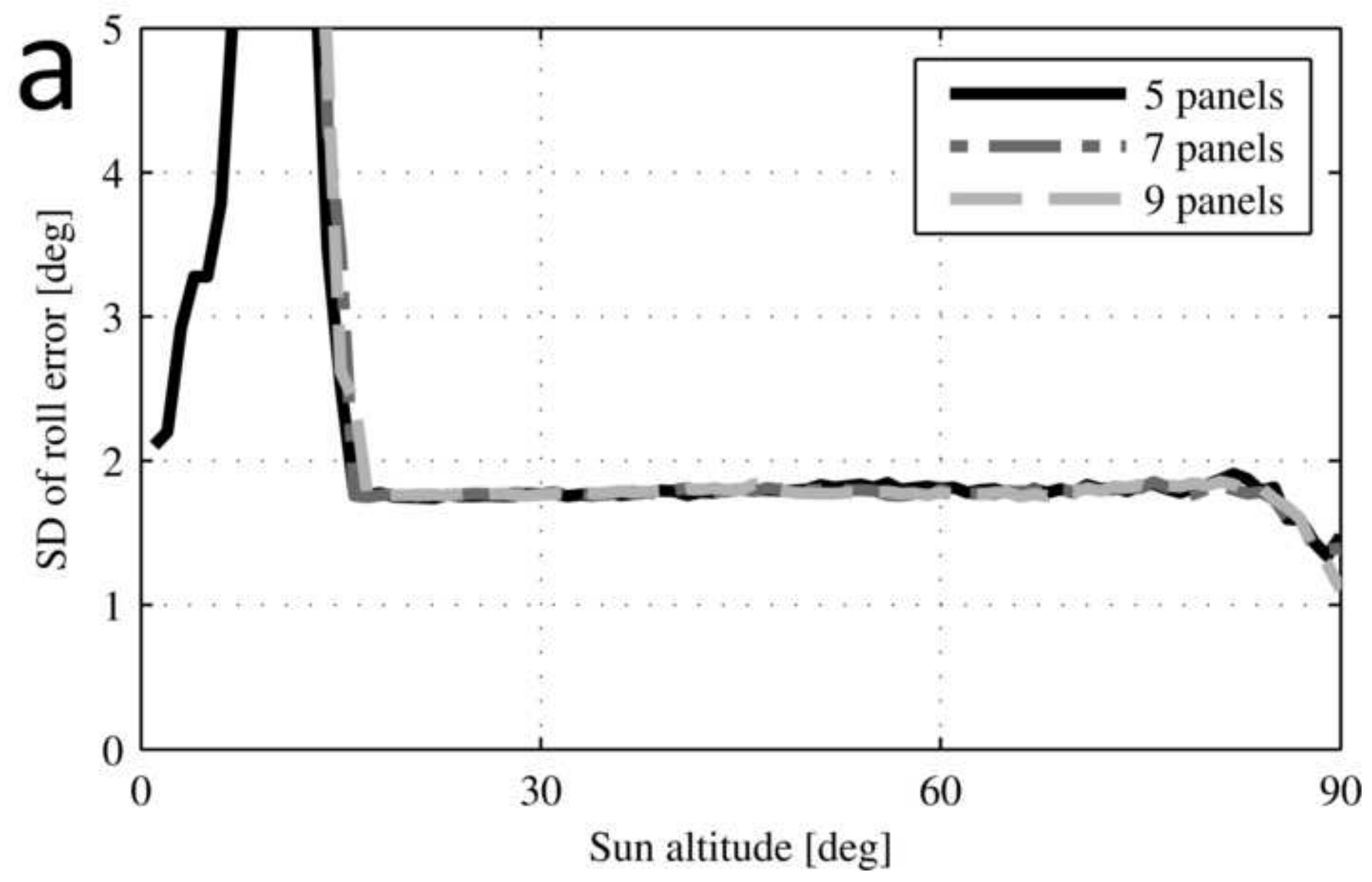




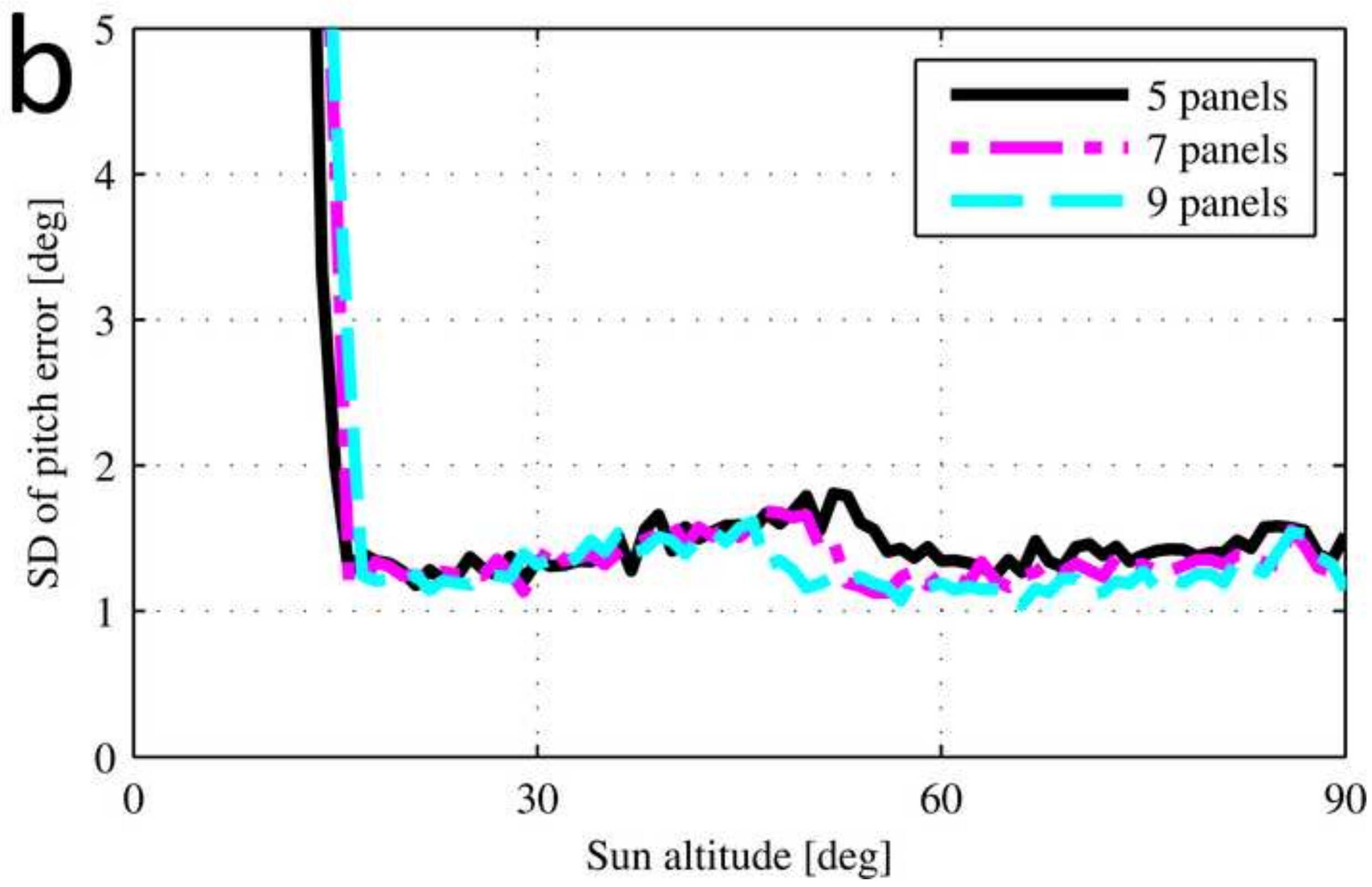




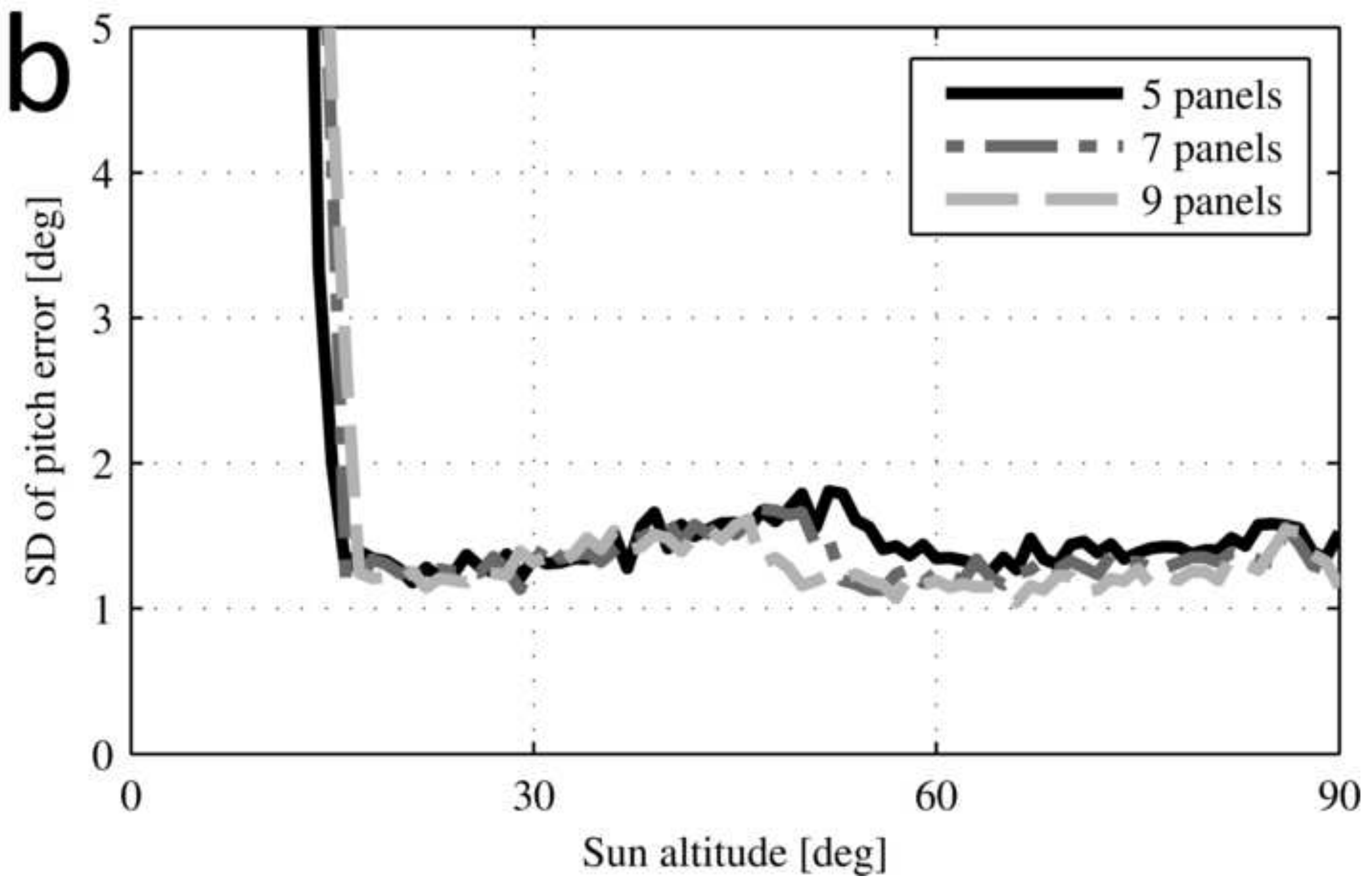



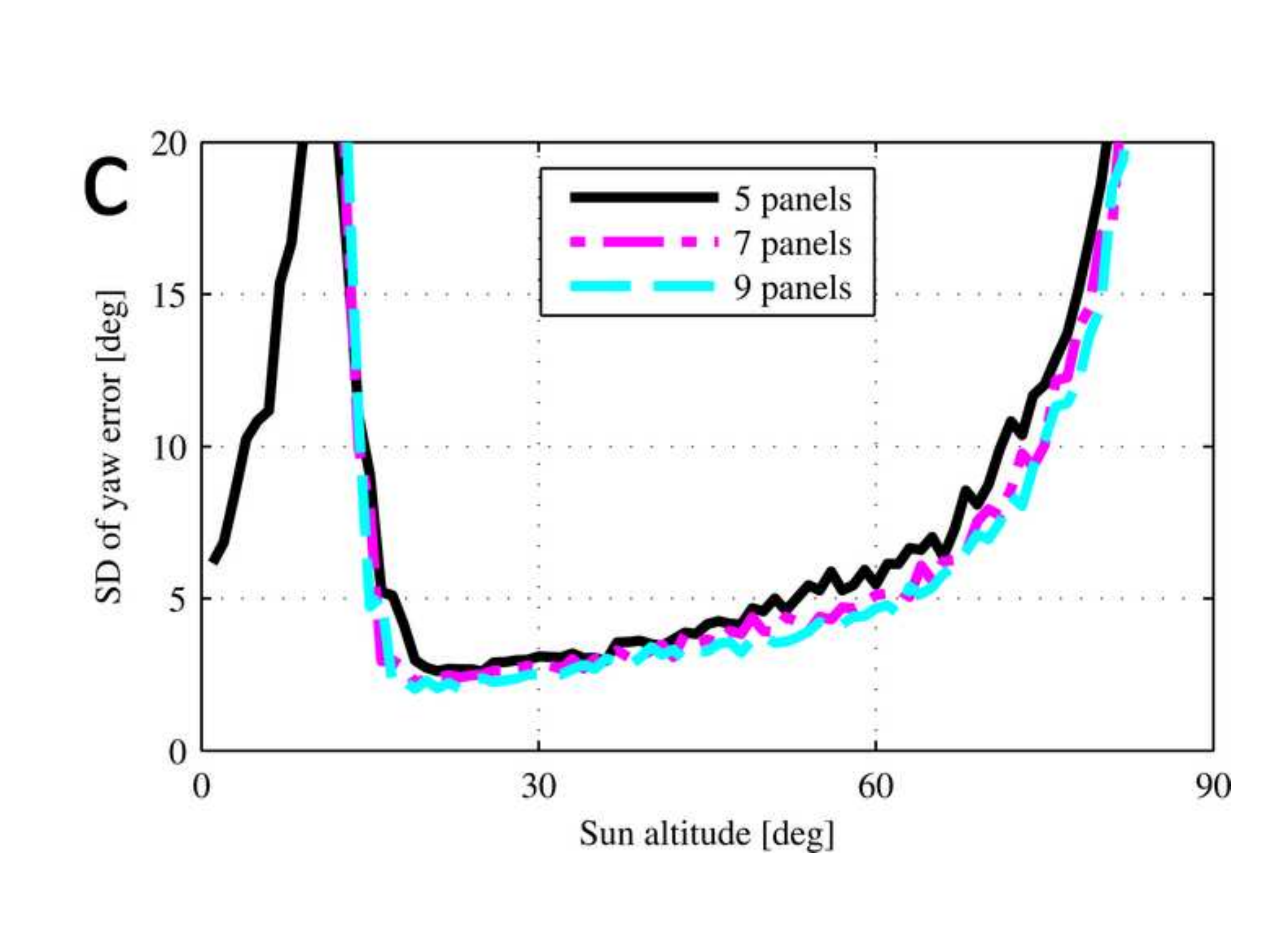


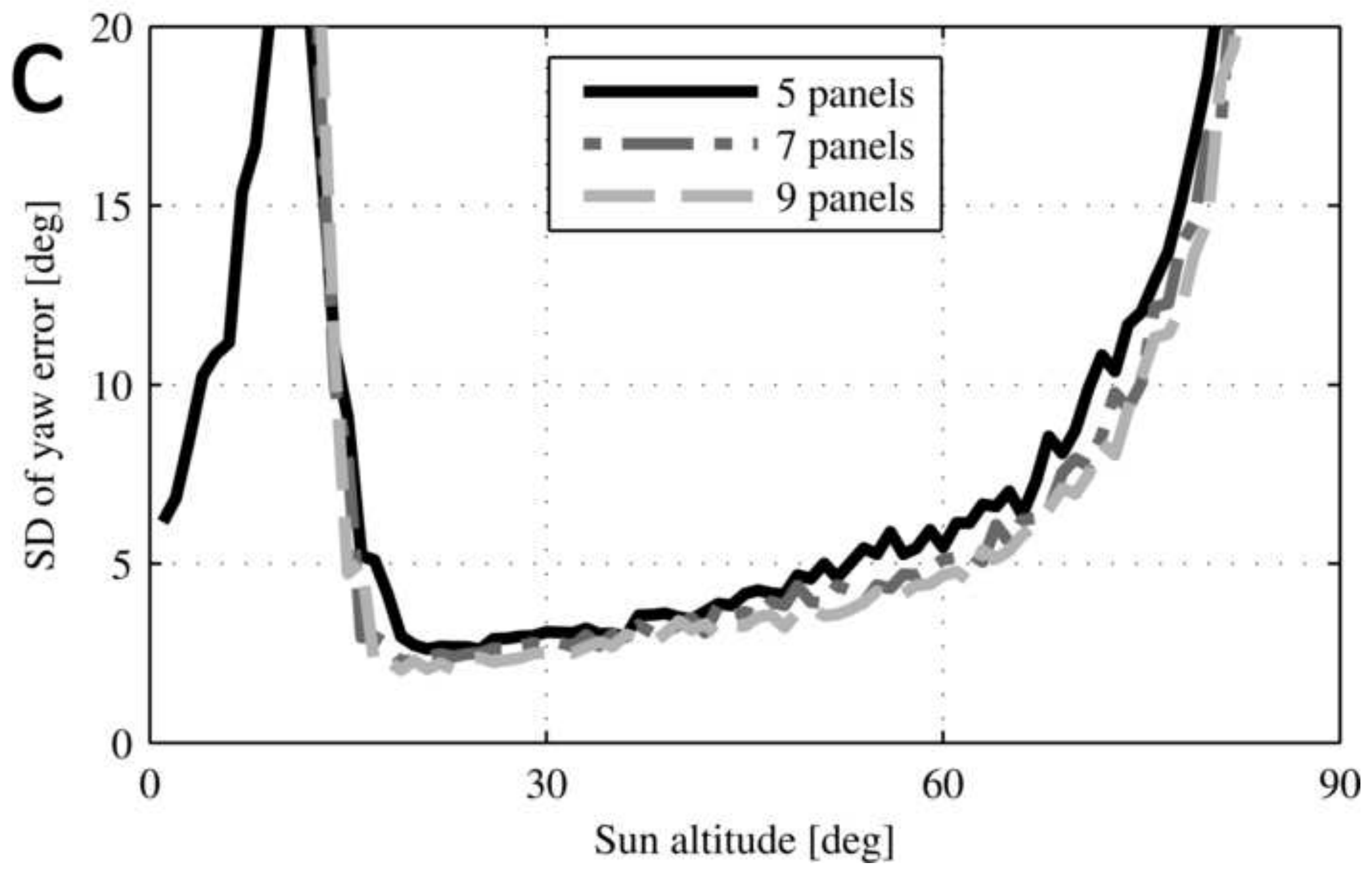


Figure $8 a$ (web)

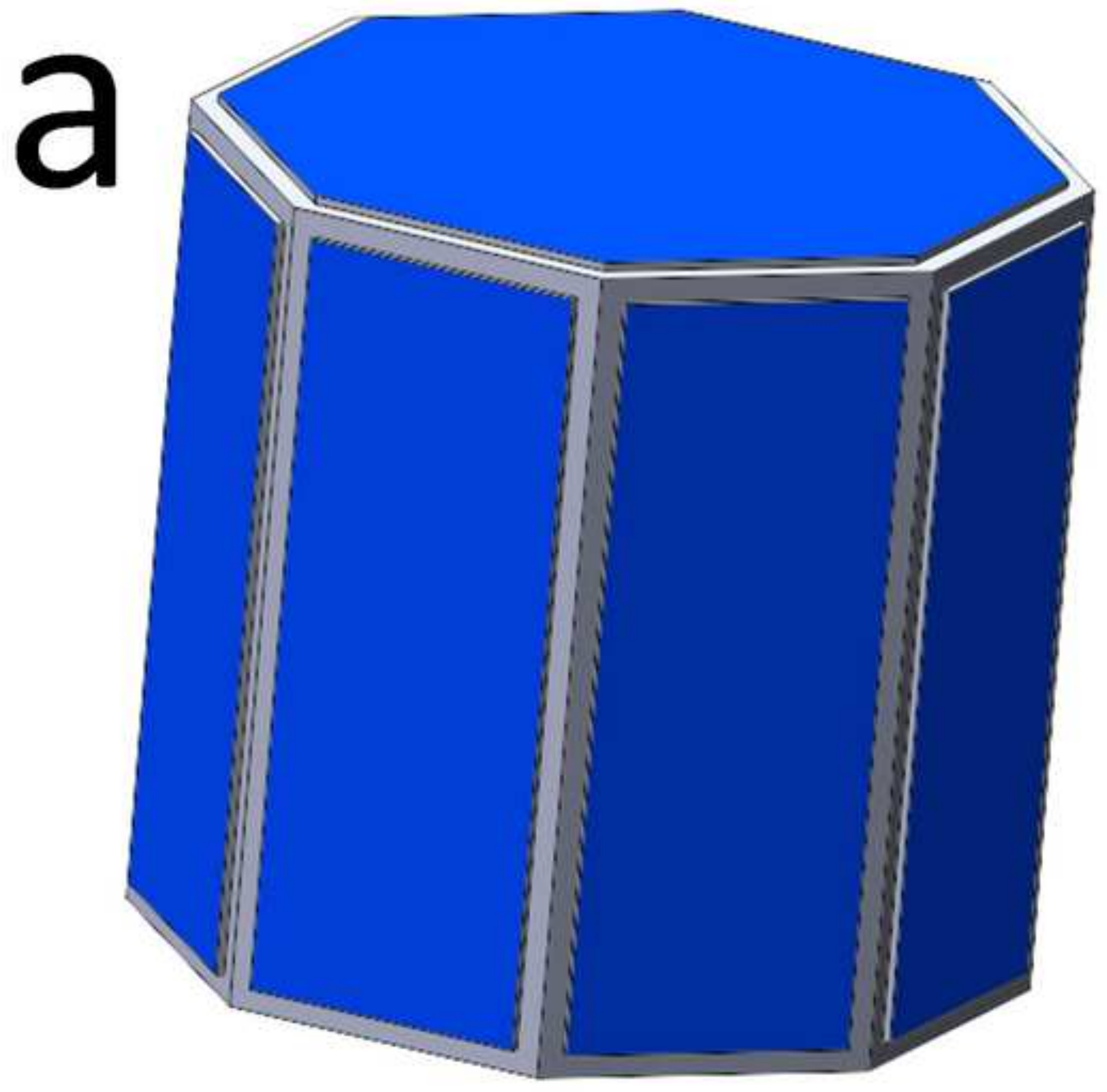


Figure $8 a$ (print)
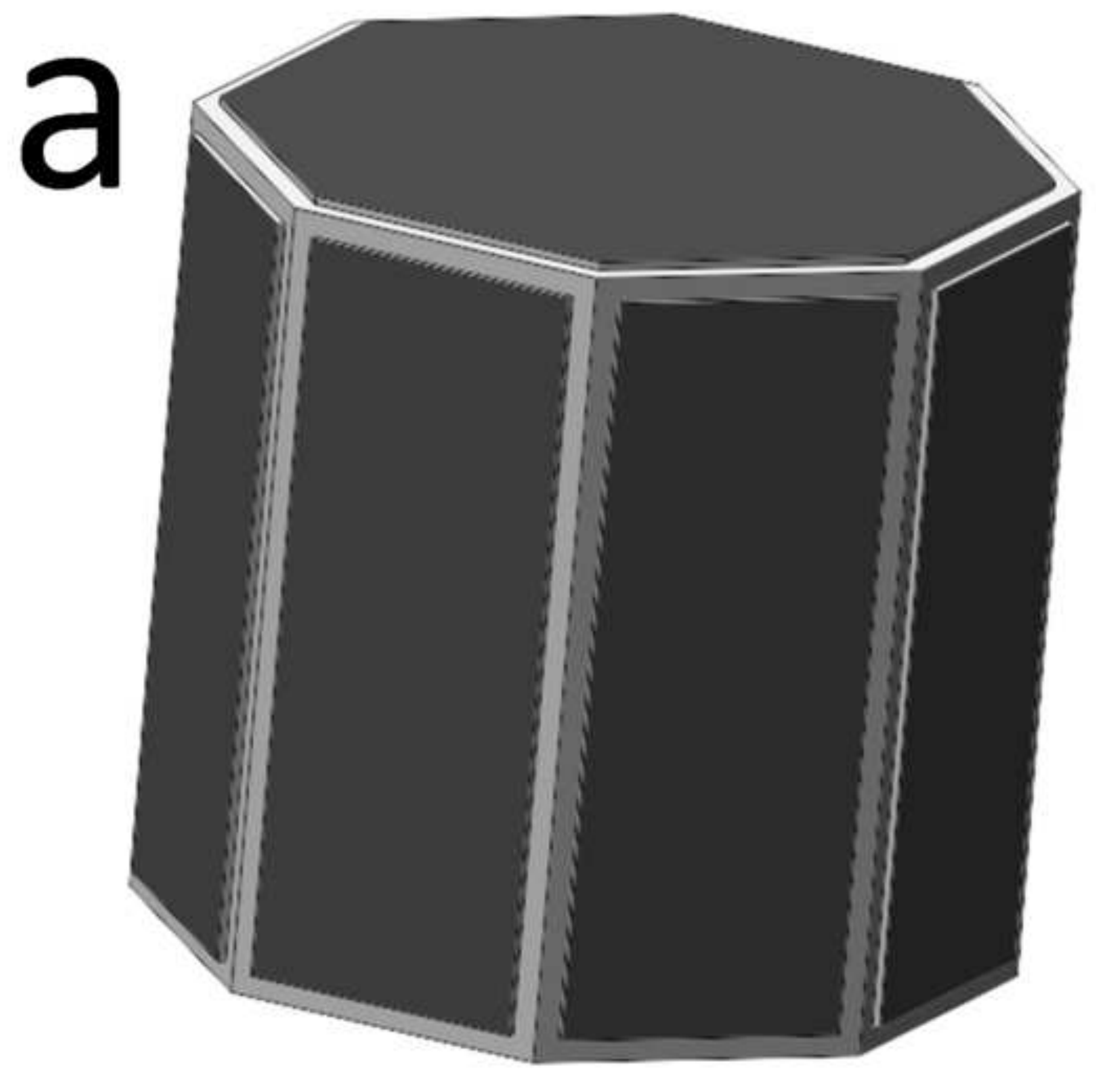

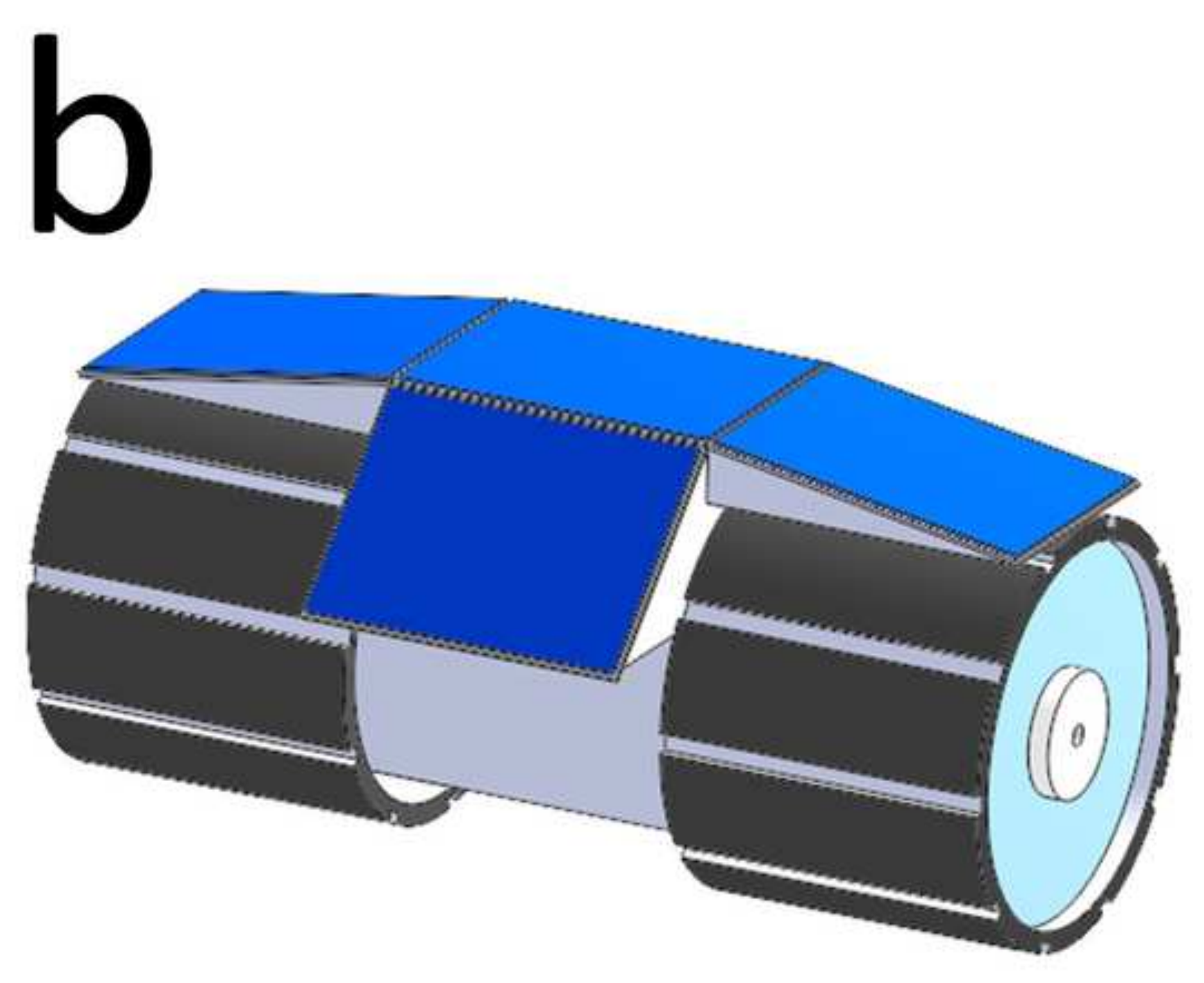

Figure $8 b$ (web)

Figure 8b (web)
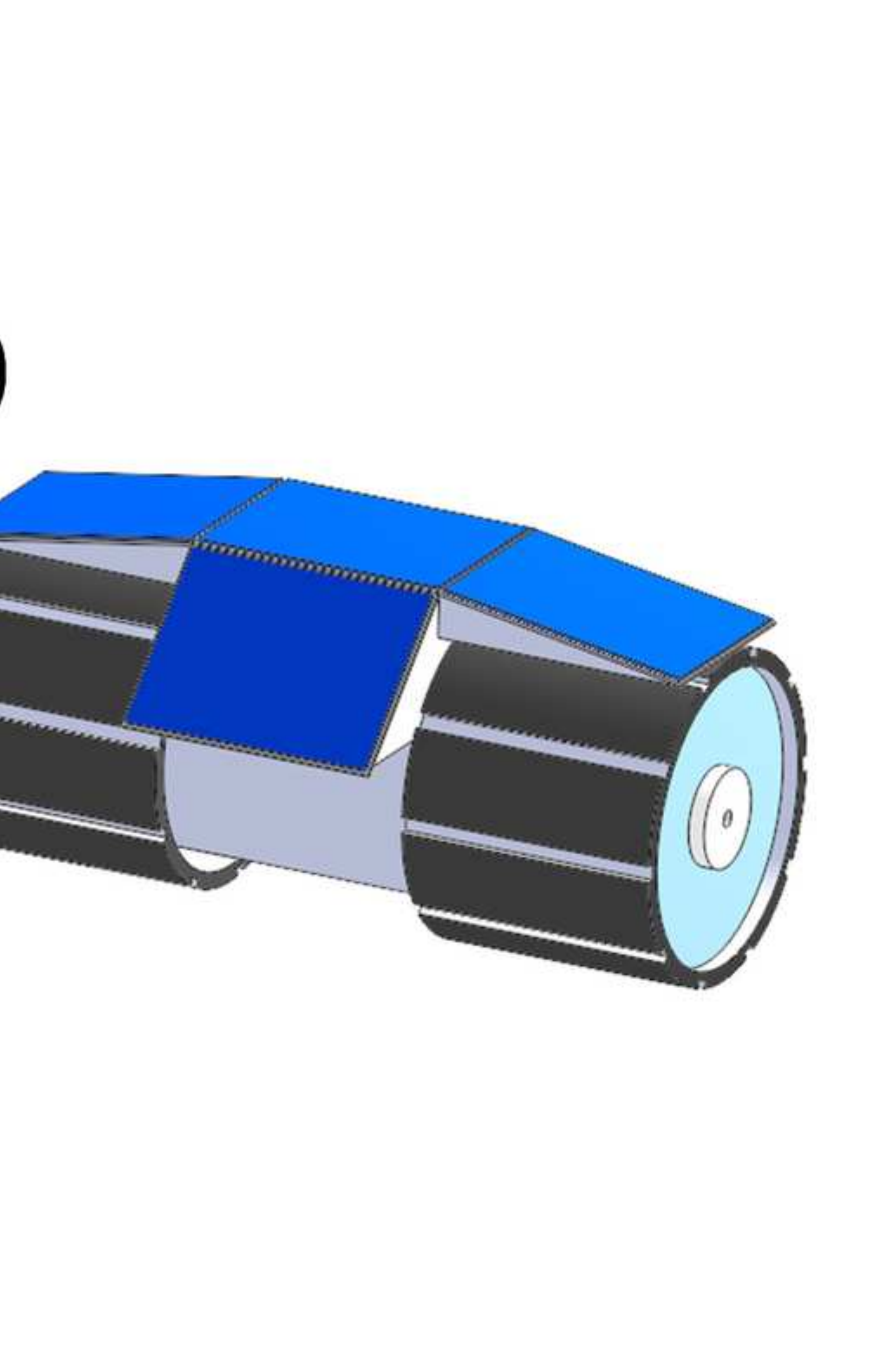

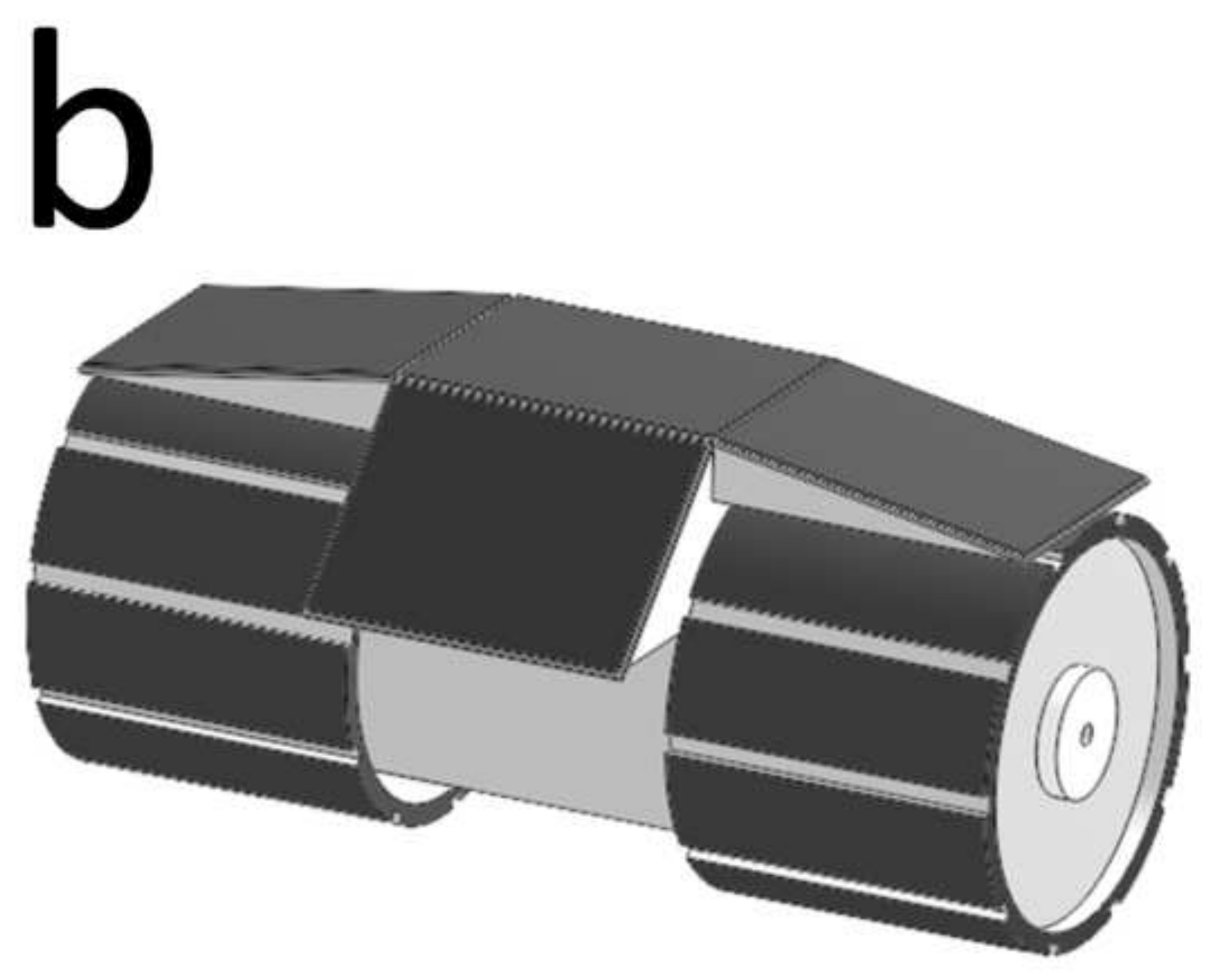

Figure $8 b$ (print)

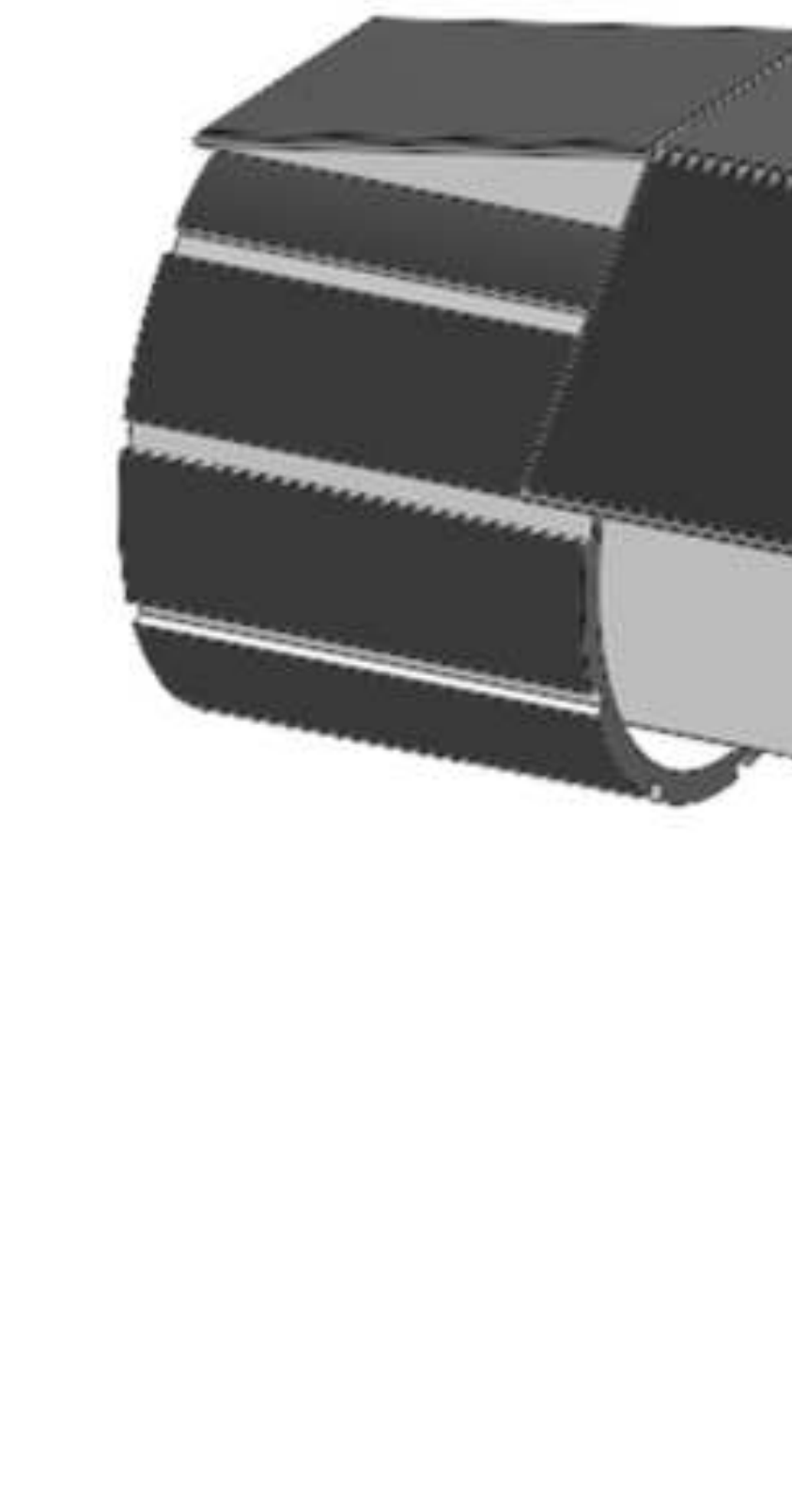

Fure $8 \mathrm{~b}$ (print) 
Figure $8 c$ (web)
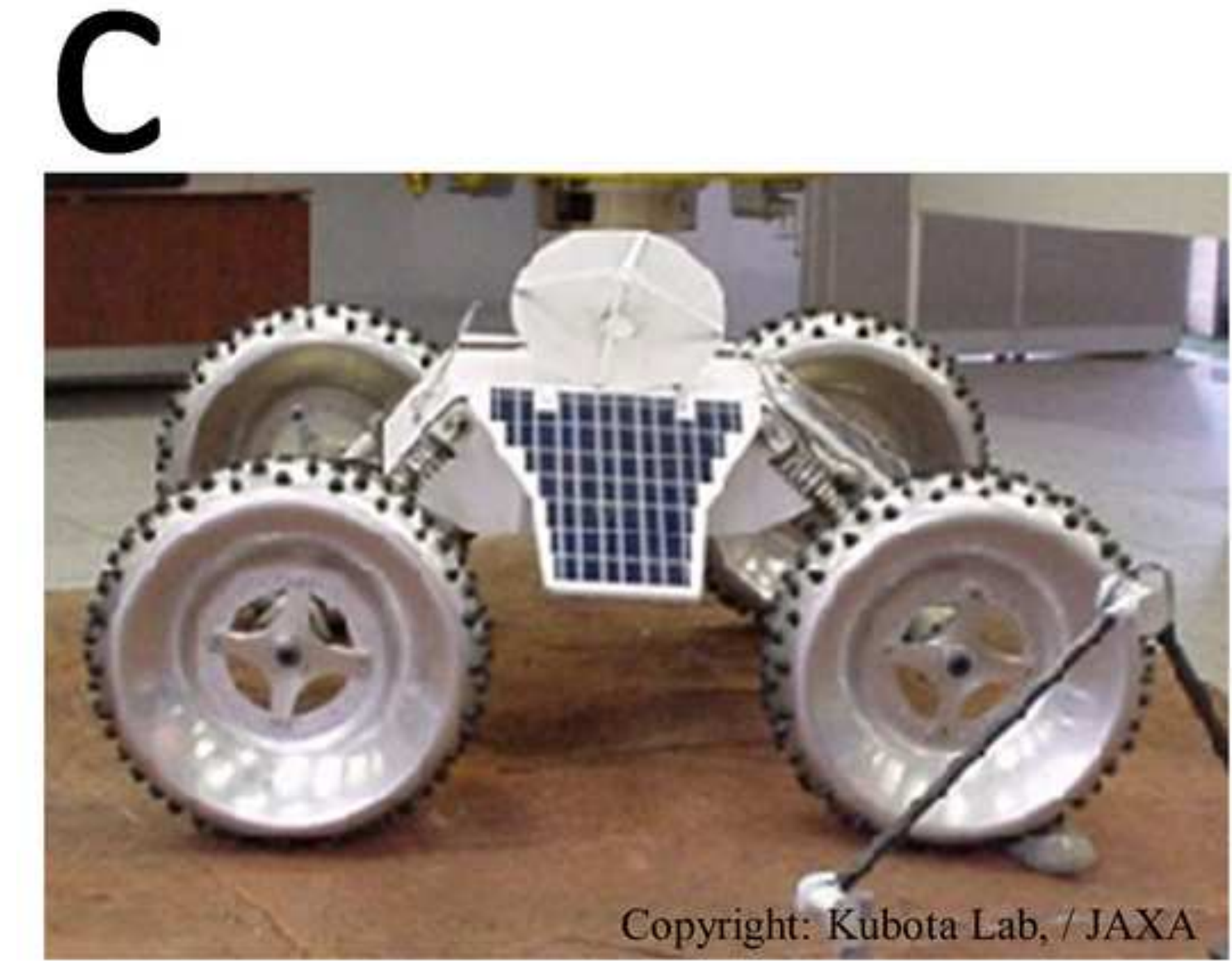

Copyright: Kubota Lab, / JAXA

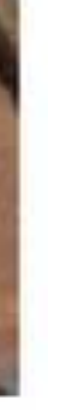

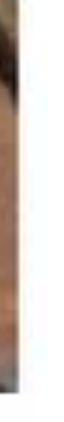
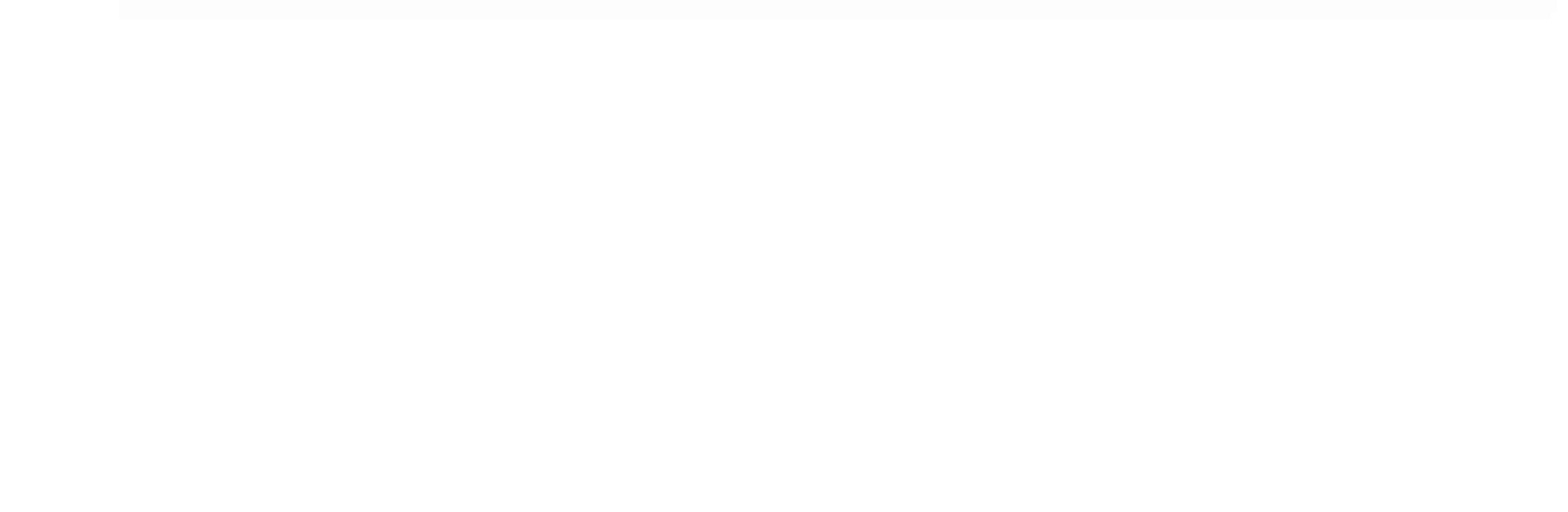

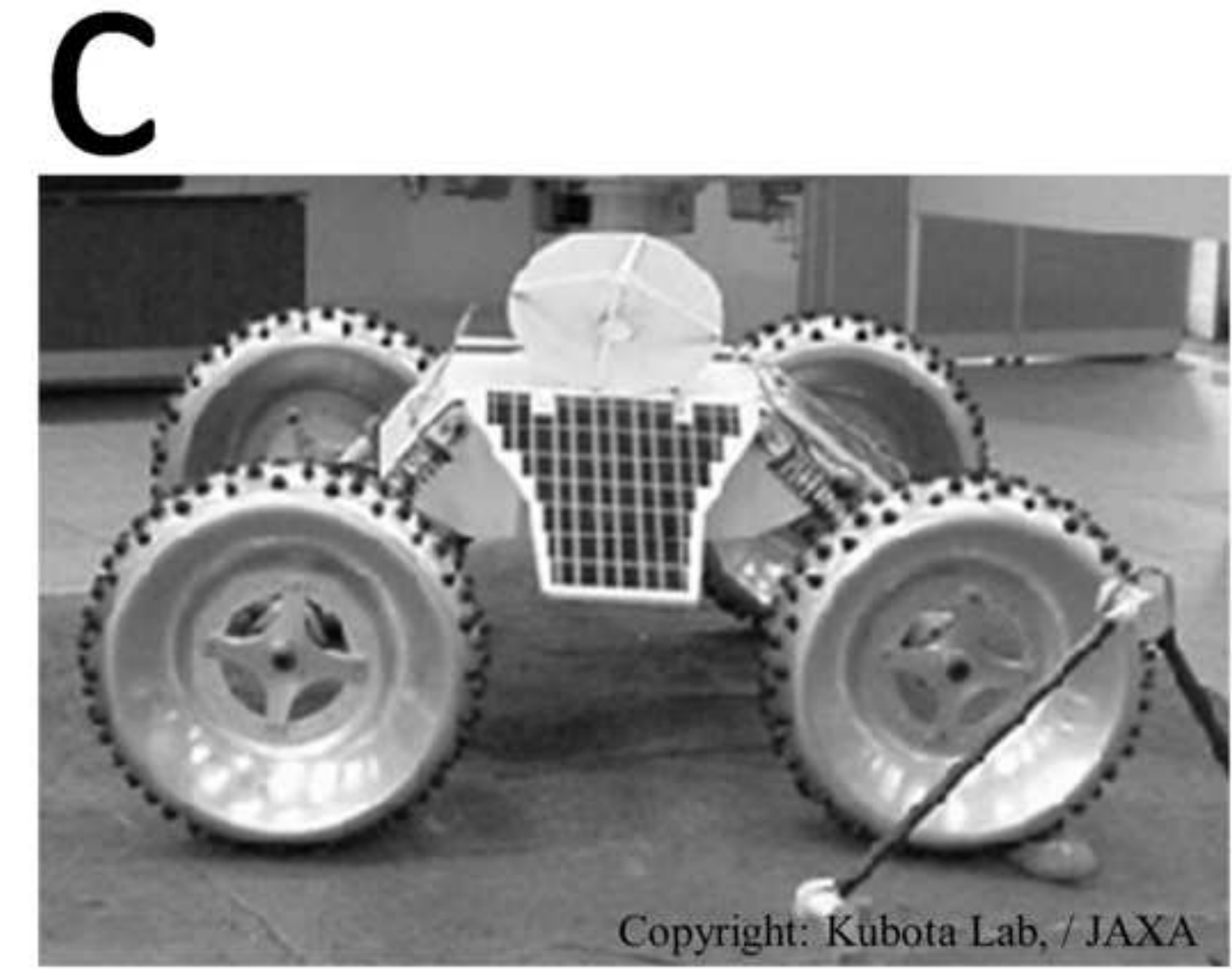

Figure $8 c$ (print)

Copyright: Kubota Lab, / JAXA

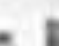

.

$$
\text { . }
$$

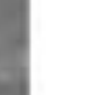

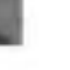

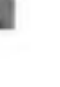



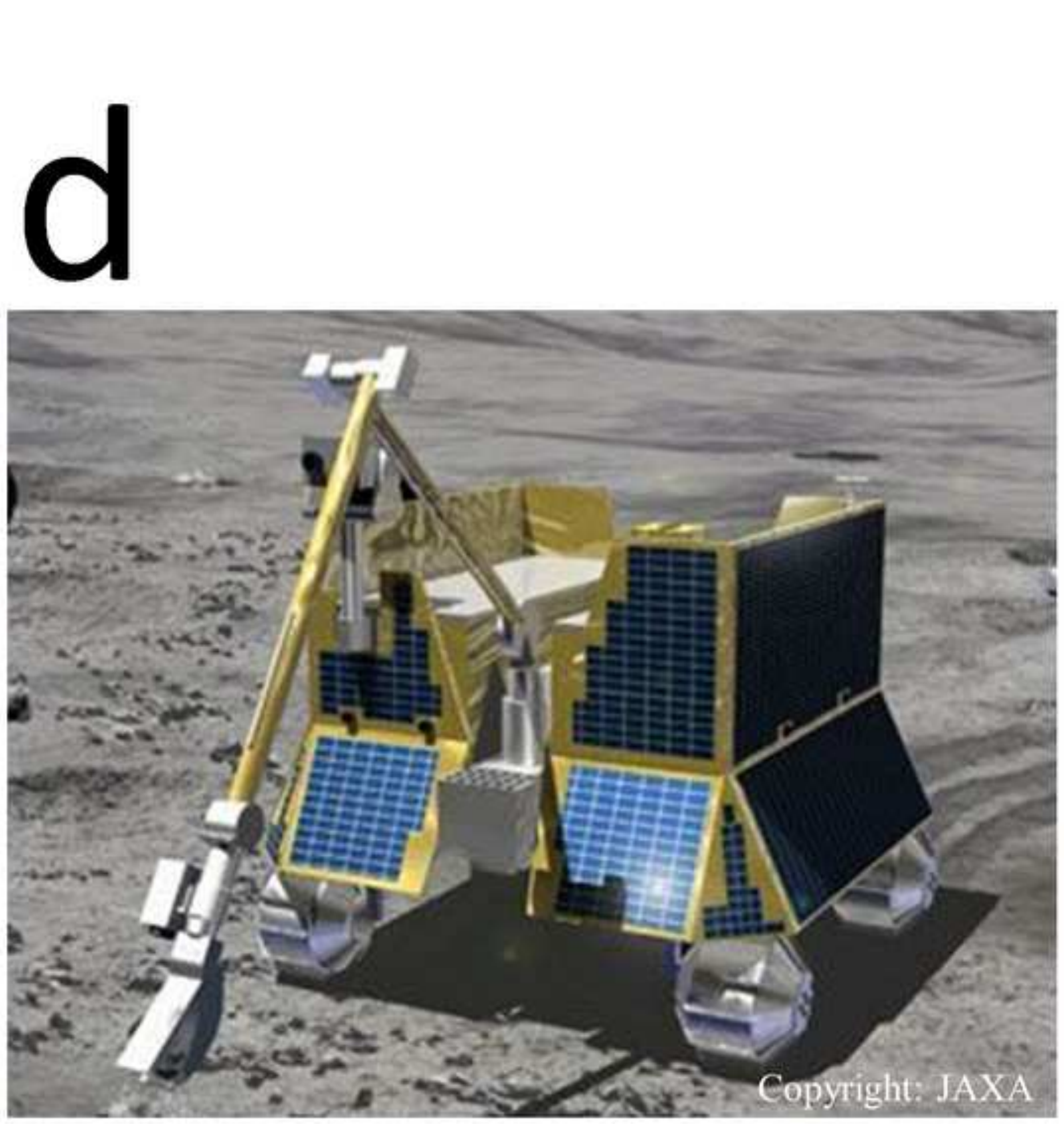

Figure $8 d$ (web)

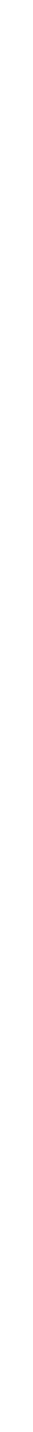

\author{
Figu (web)
}

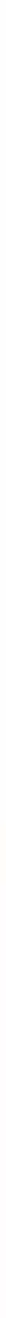
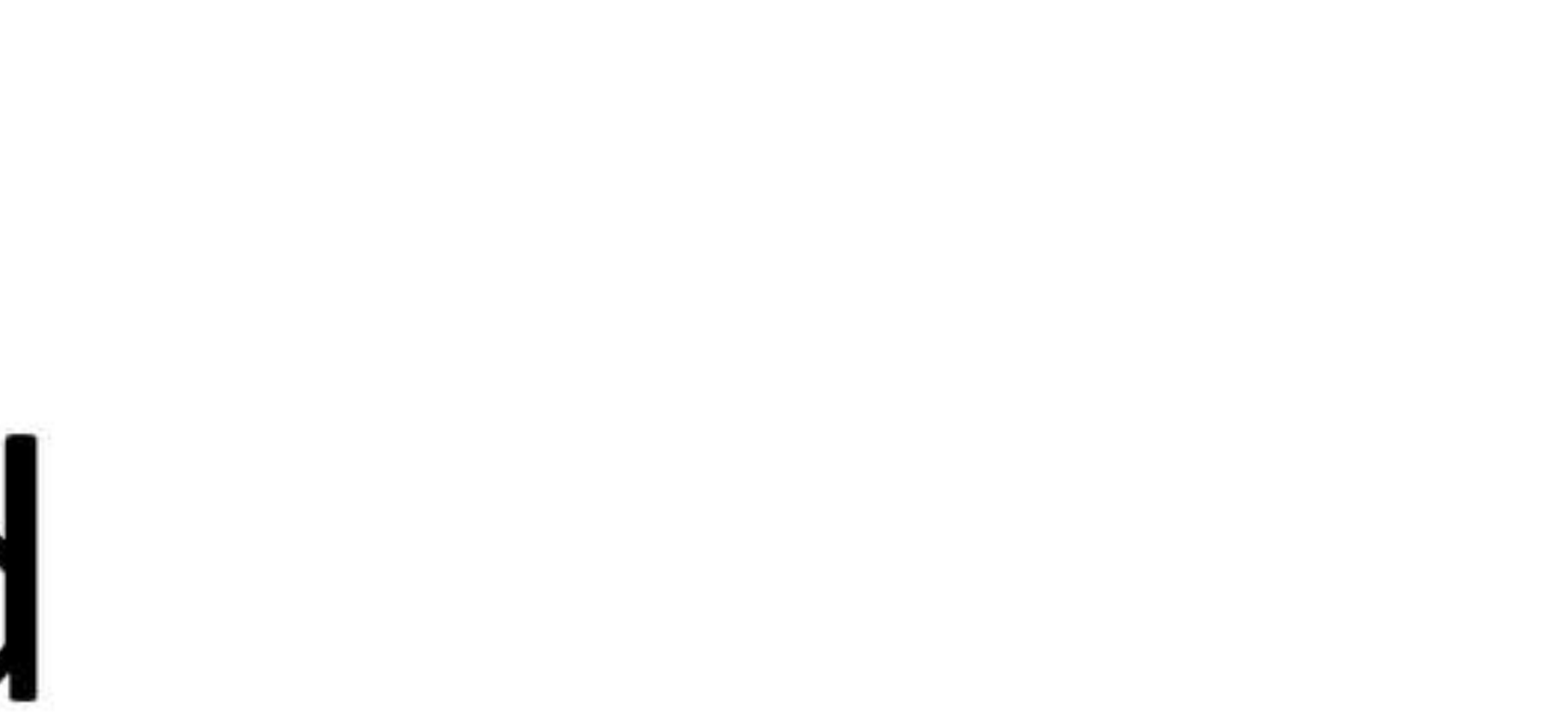

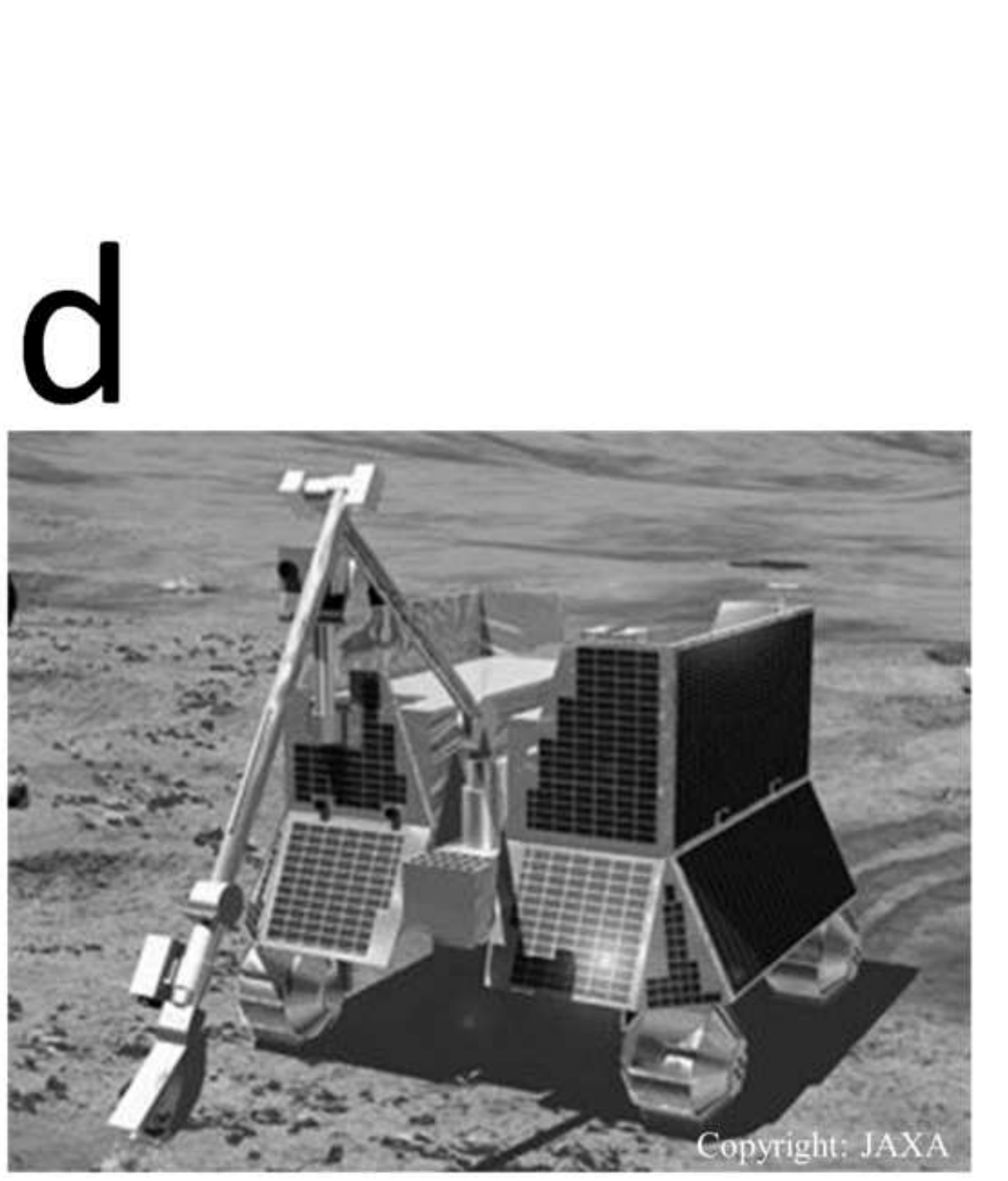

Figure $8 d$ (print)

\section{Figure $8 d$ (print)}
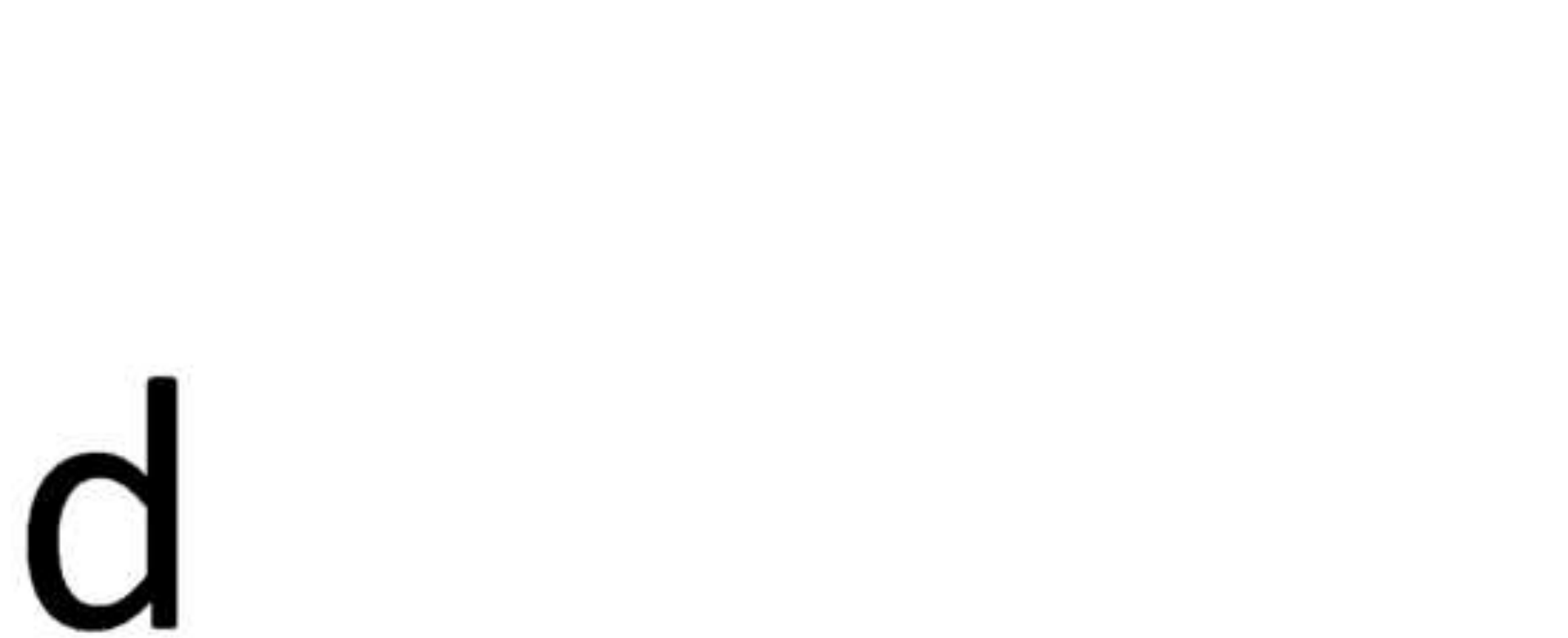

(

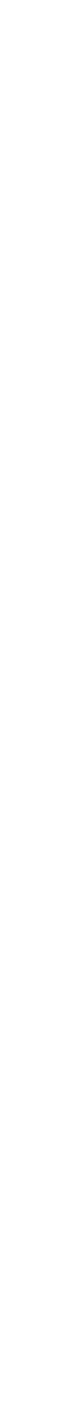

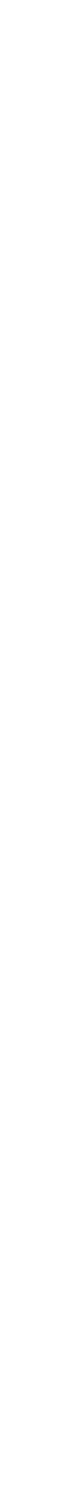




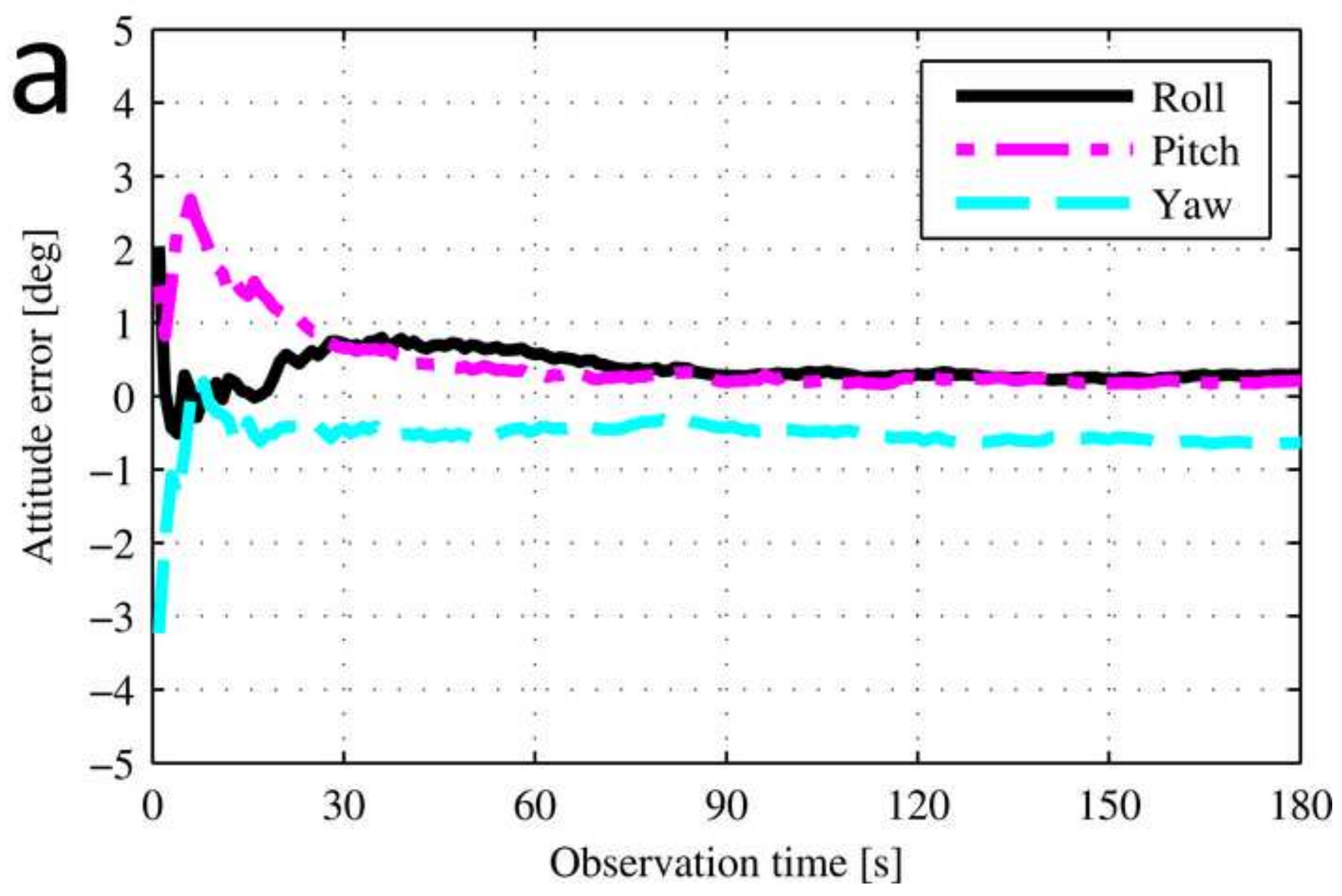




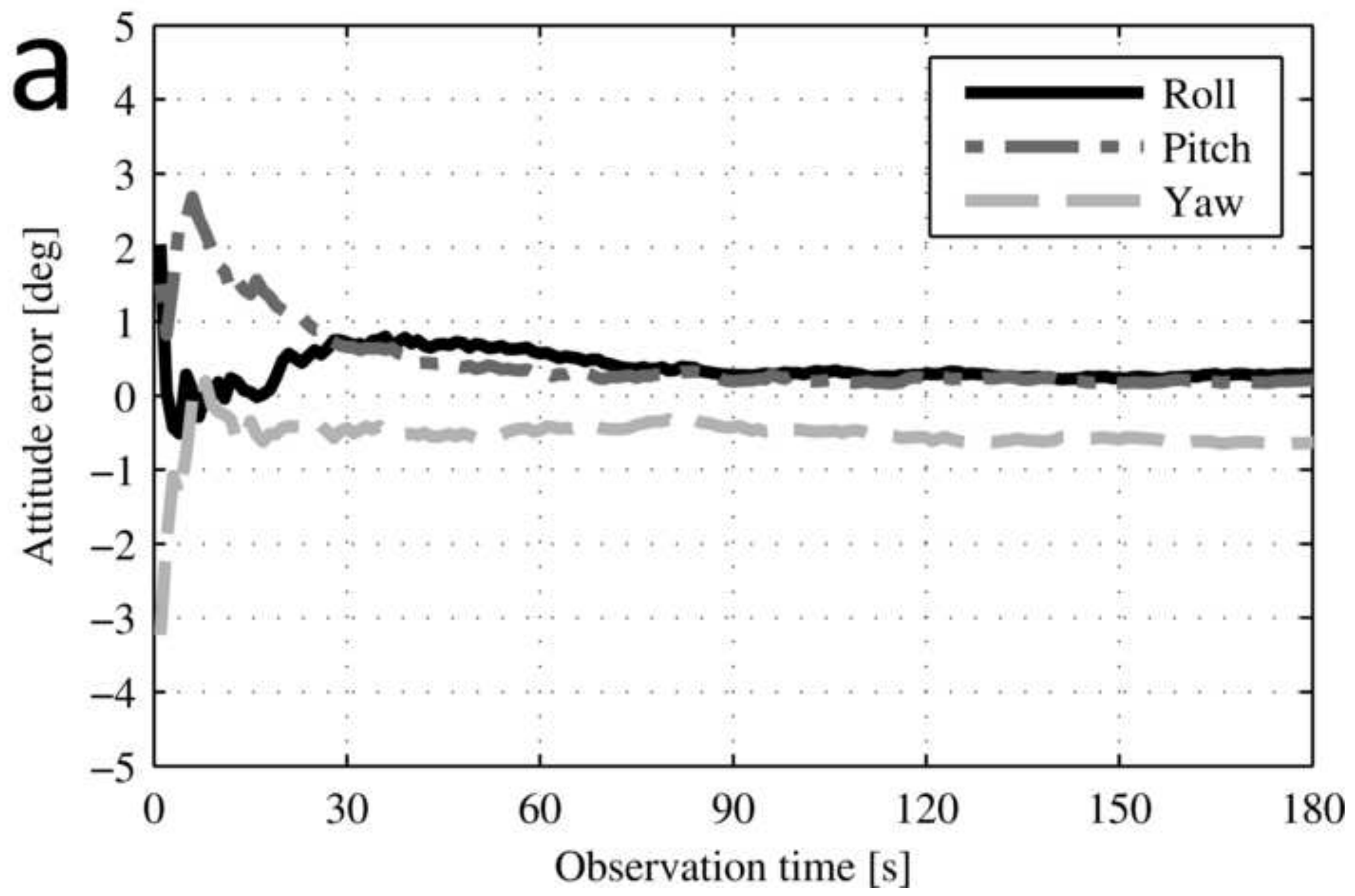




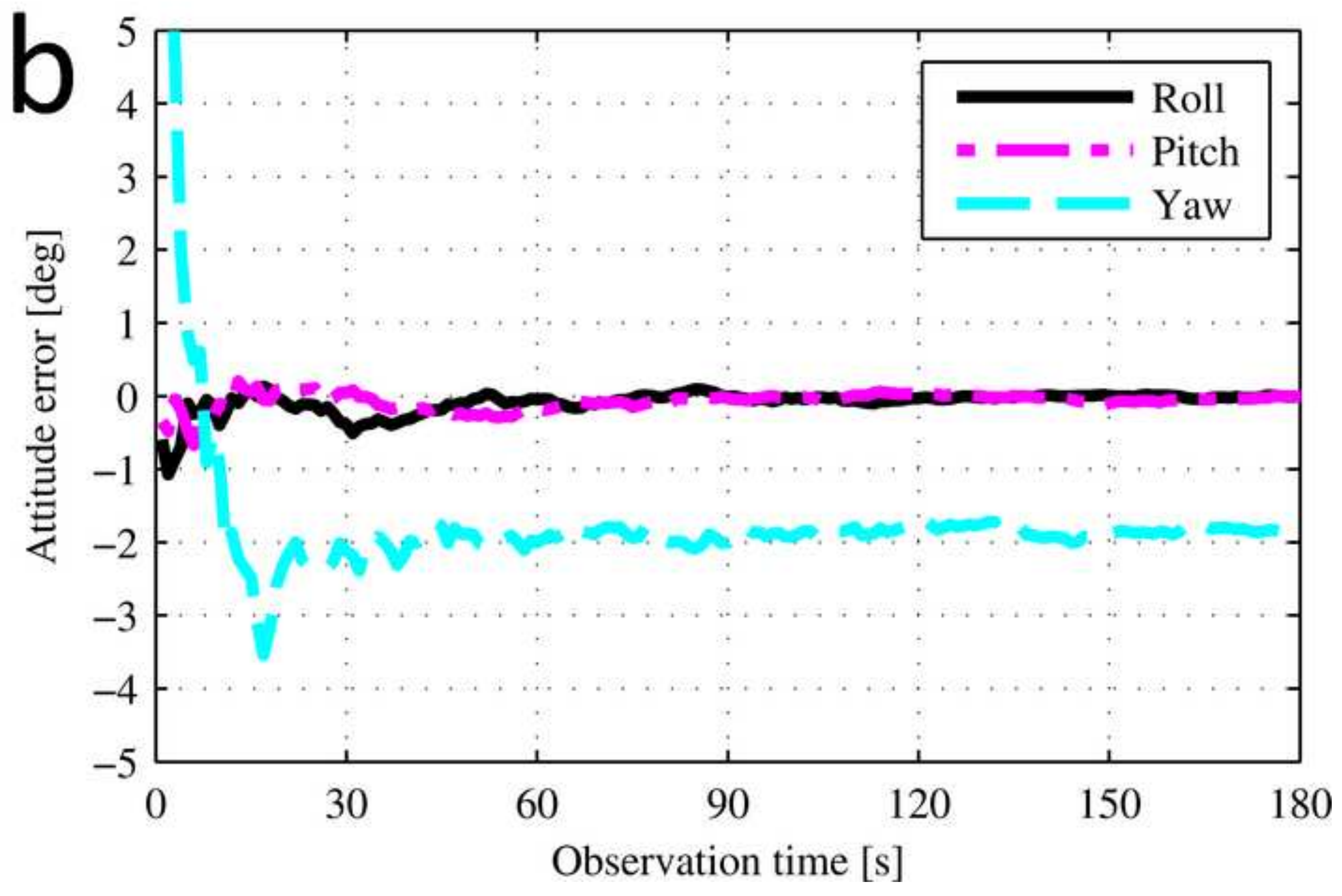




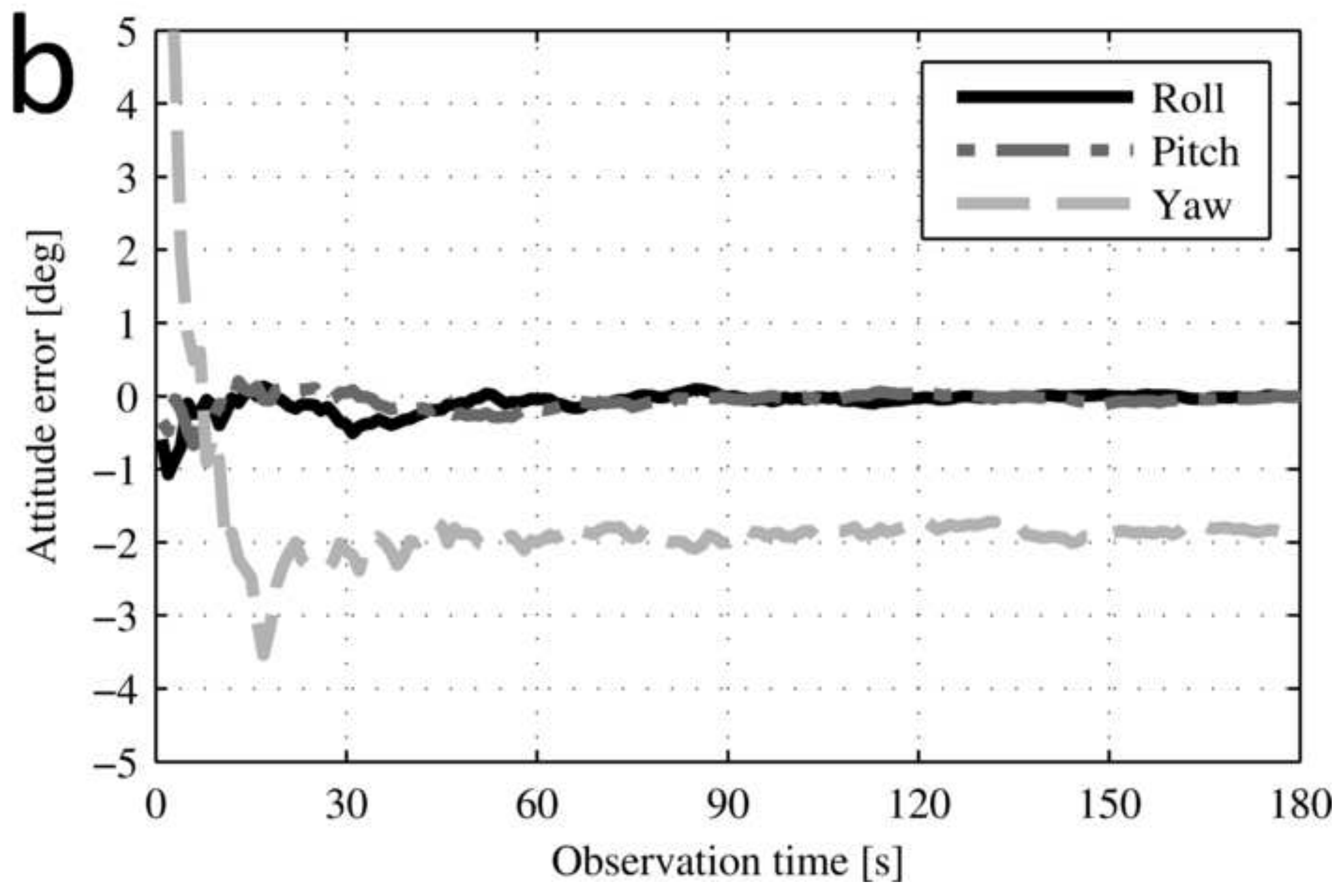




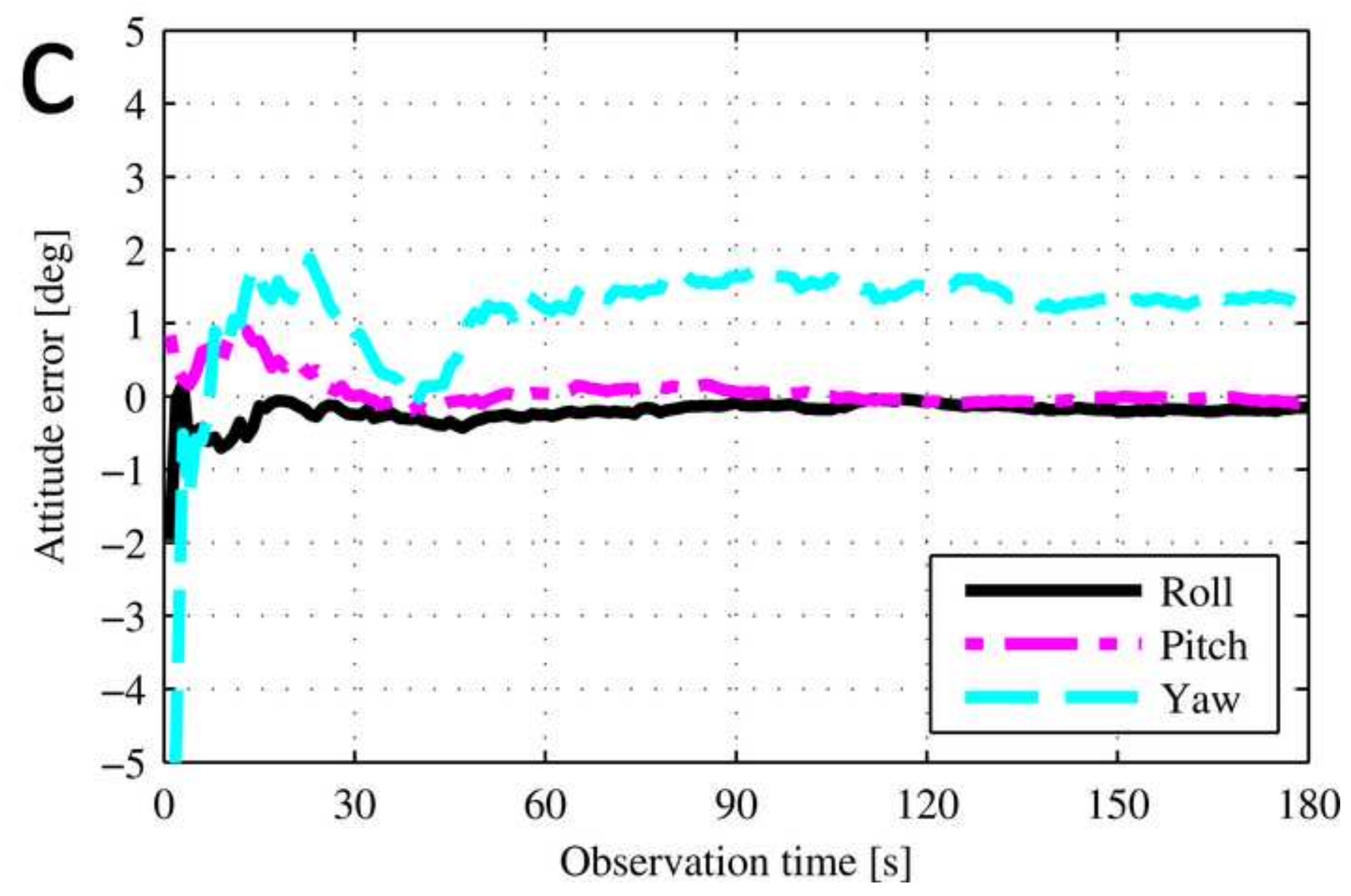




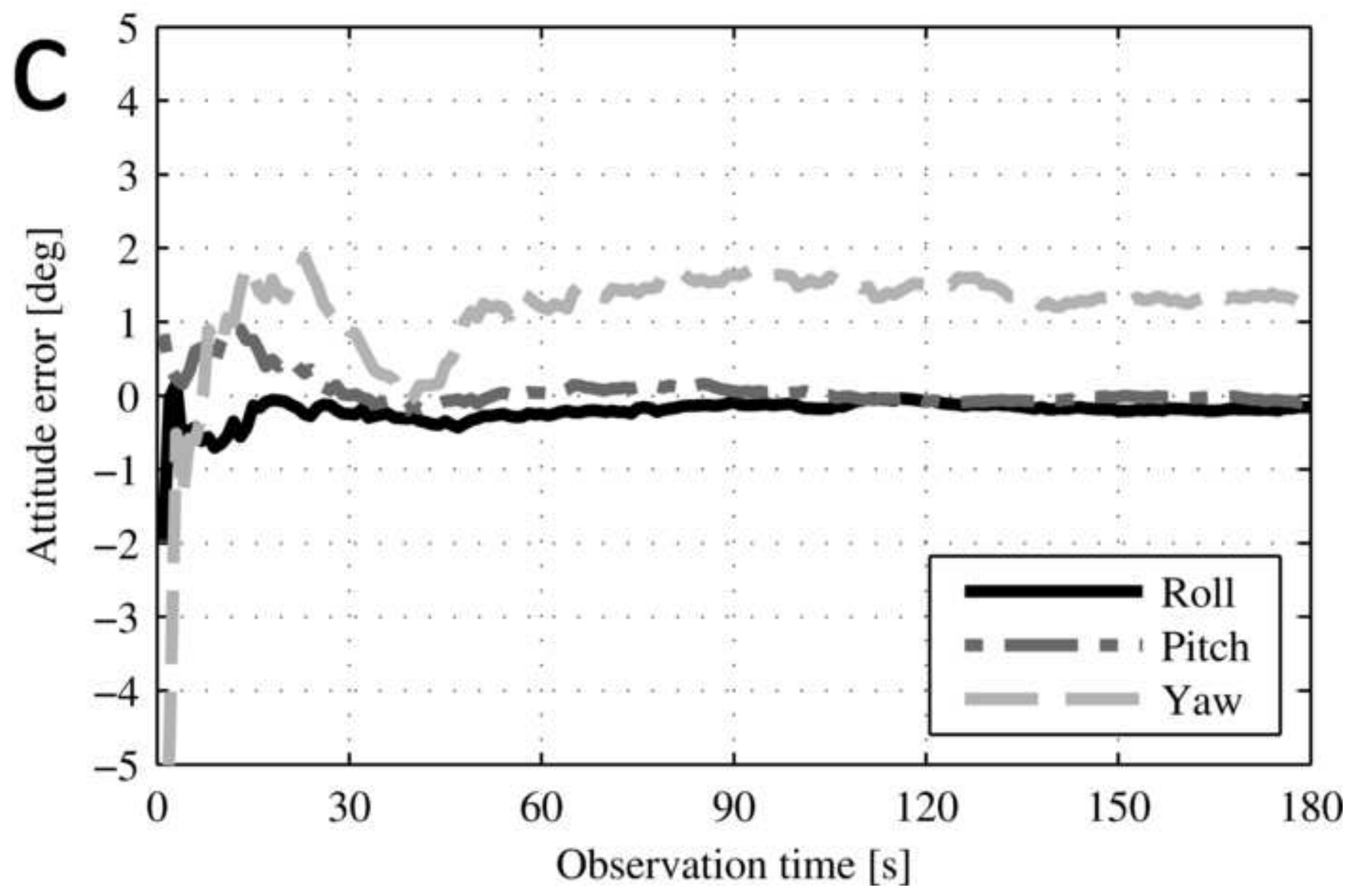




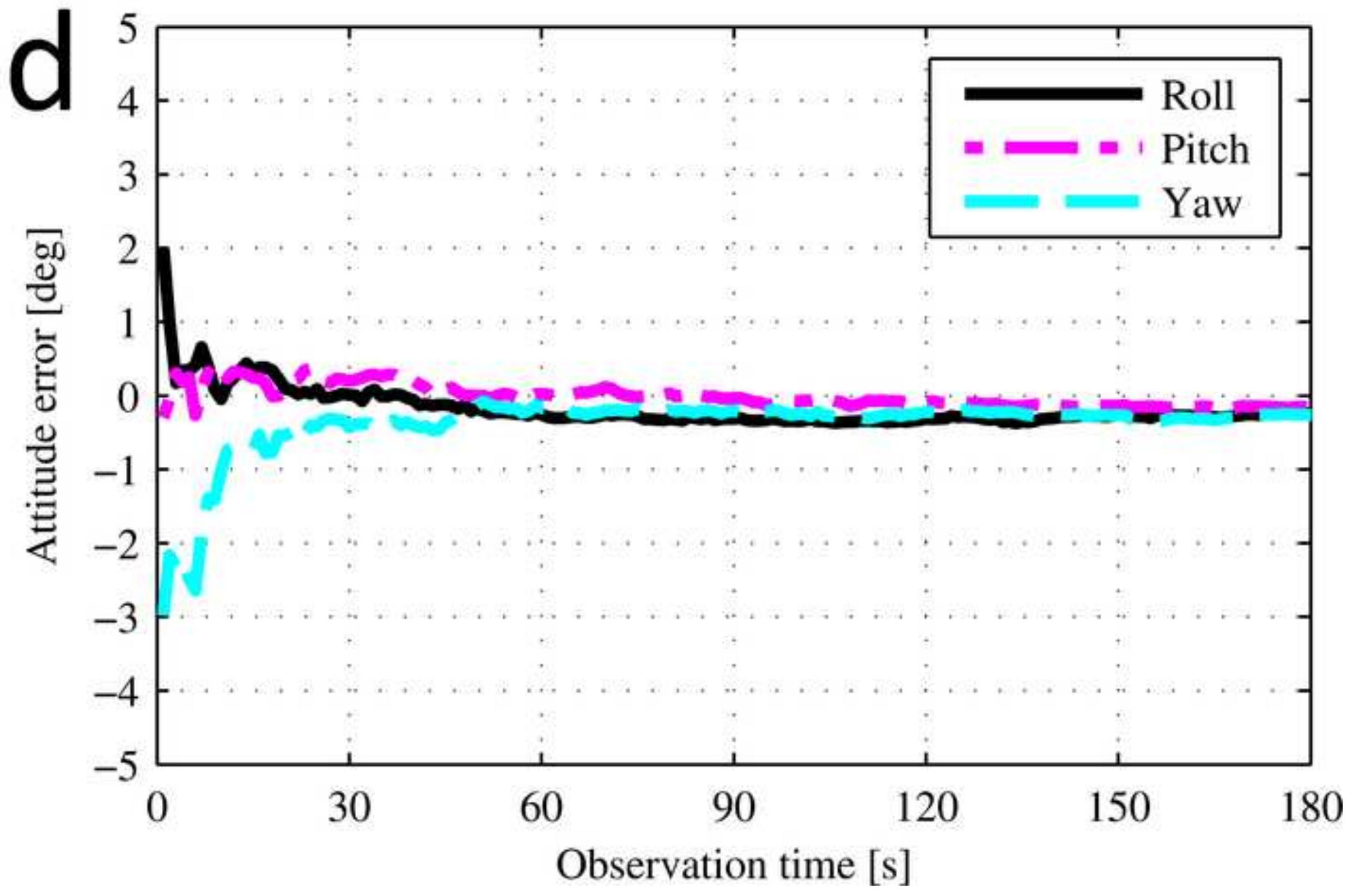




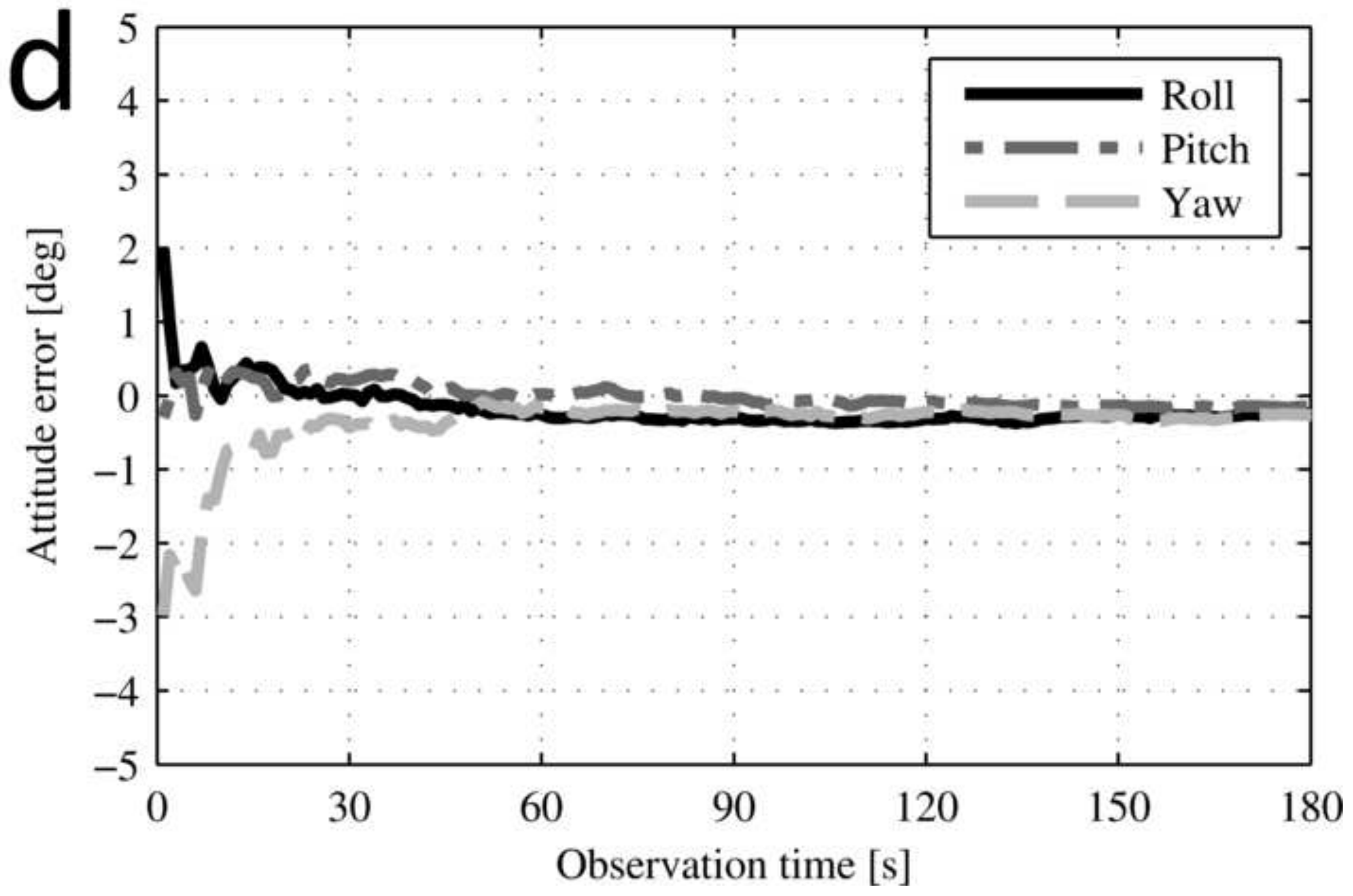



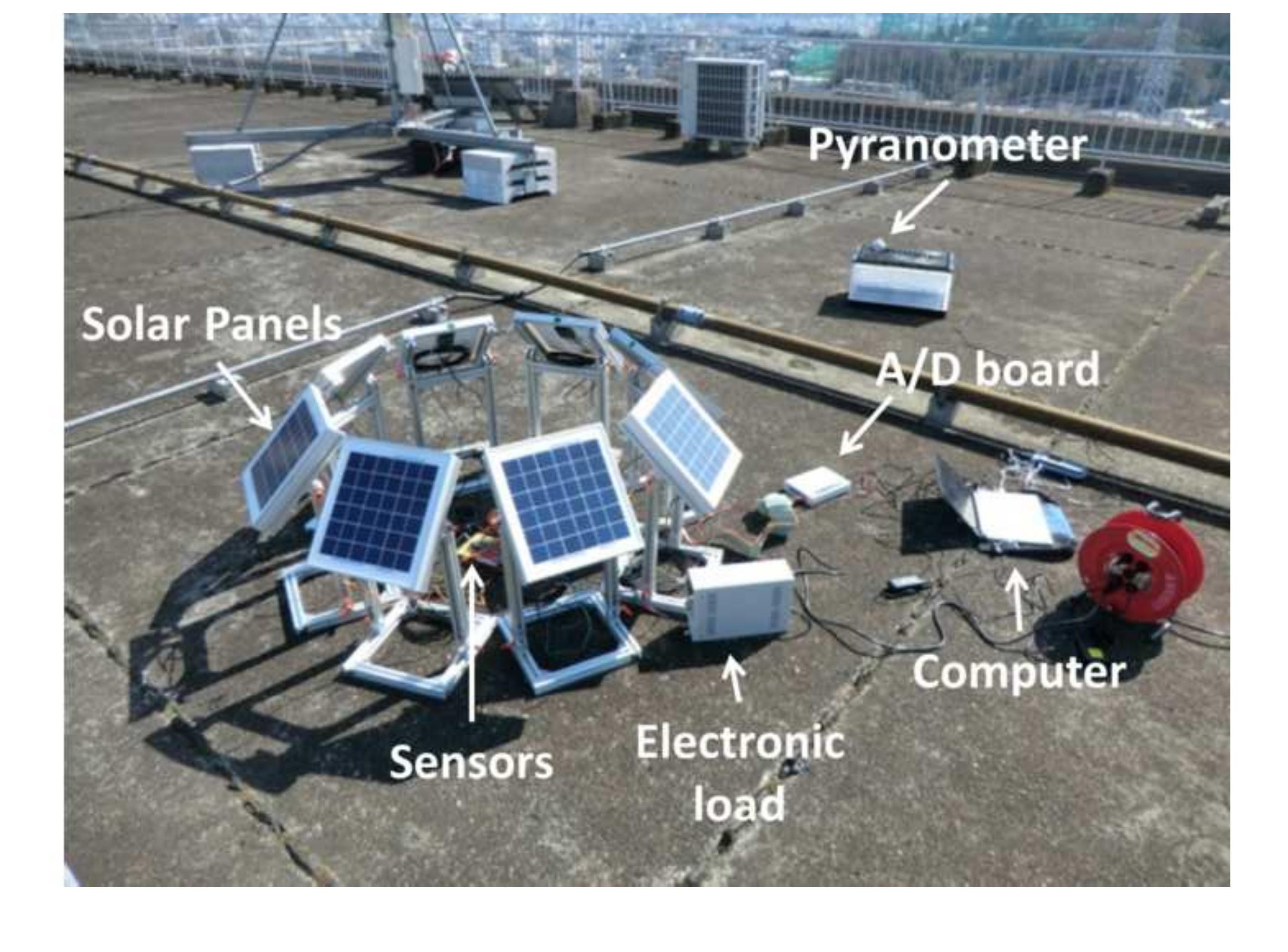

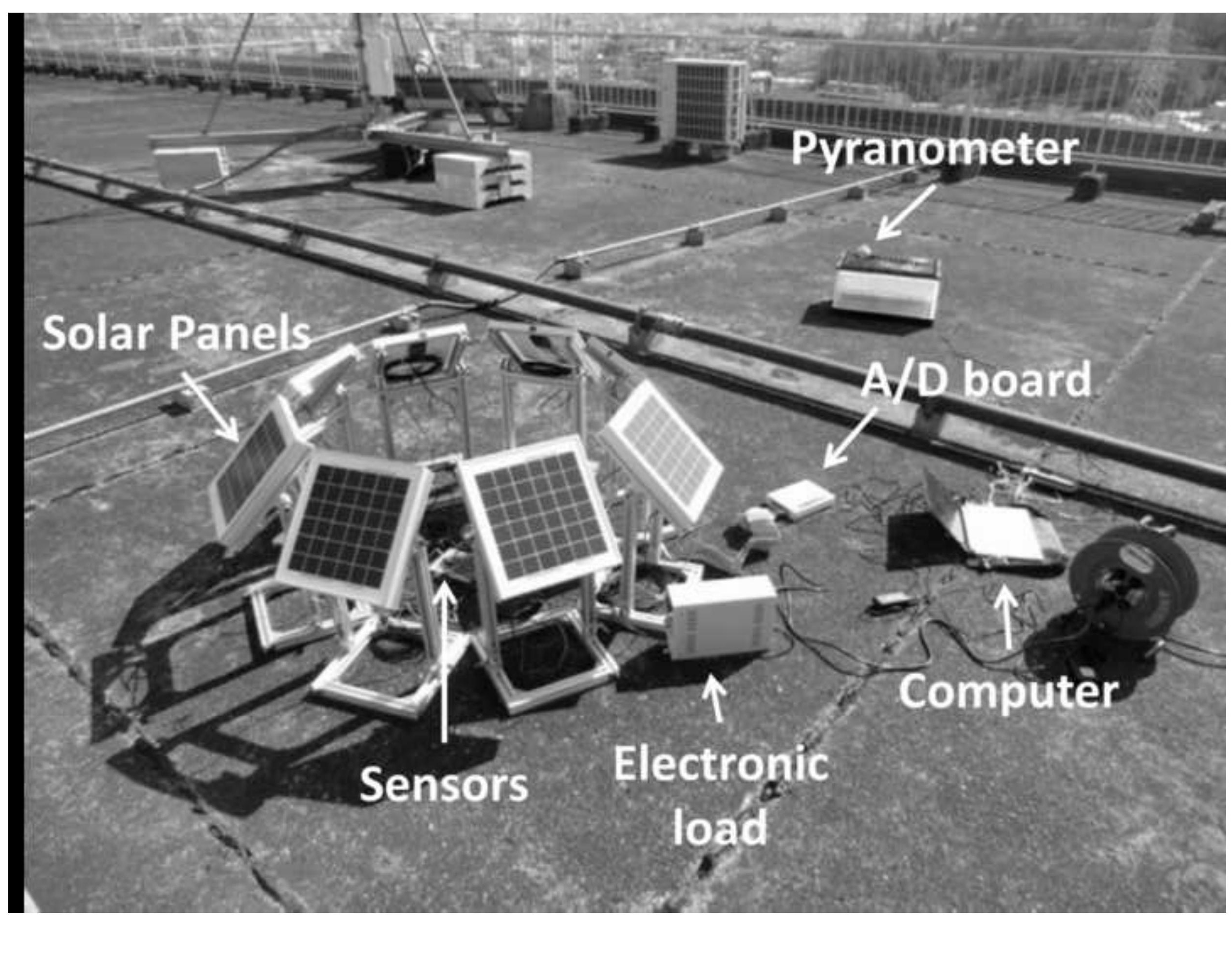


$$
\mathrm{SP} 1 \longrightarrow \mathrm{SP} 2 \longrightarrow \mathrm{SP} 3 \longrightarrow \mathrm{SP} 4
$$

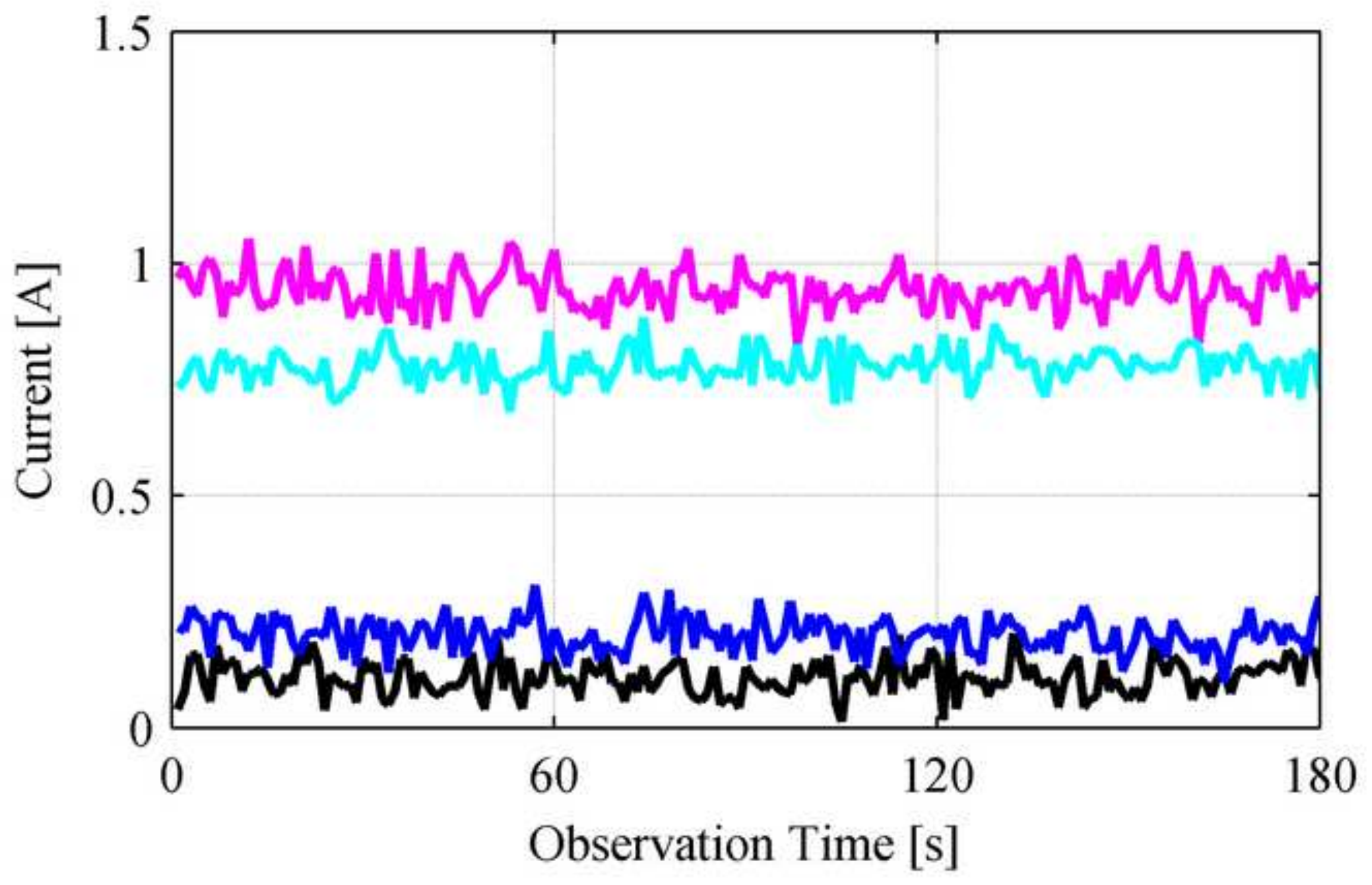




$$
\longrightarrow \mathrm{SP} 1 \longrightarrow \mathrm{SP} 2 \longrightarrow \mathrm{SP} 3 \longrightarrow \mathrm{SP} 4
$$

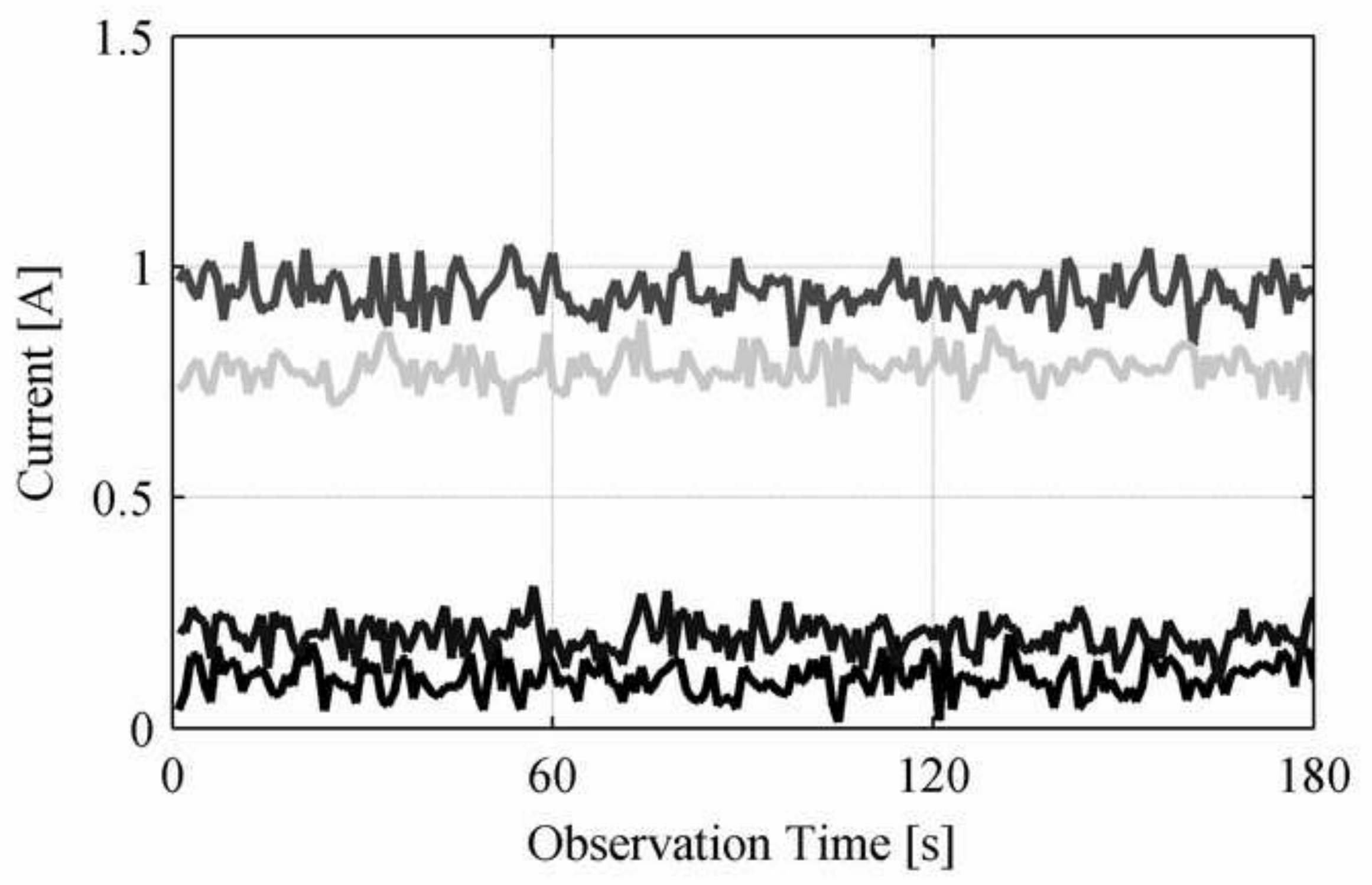




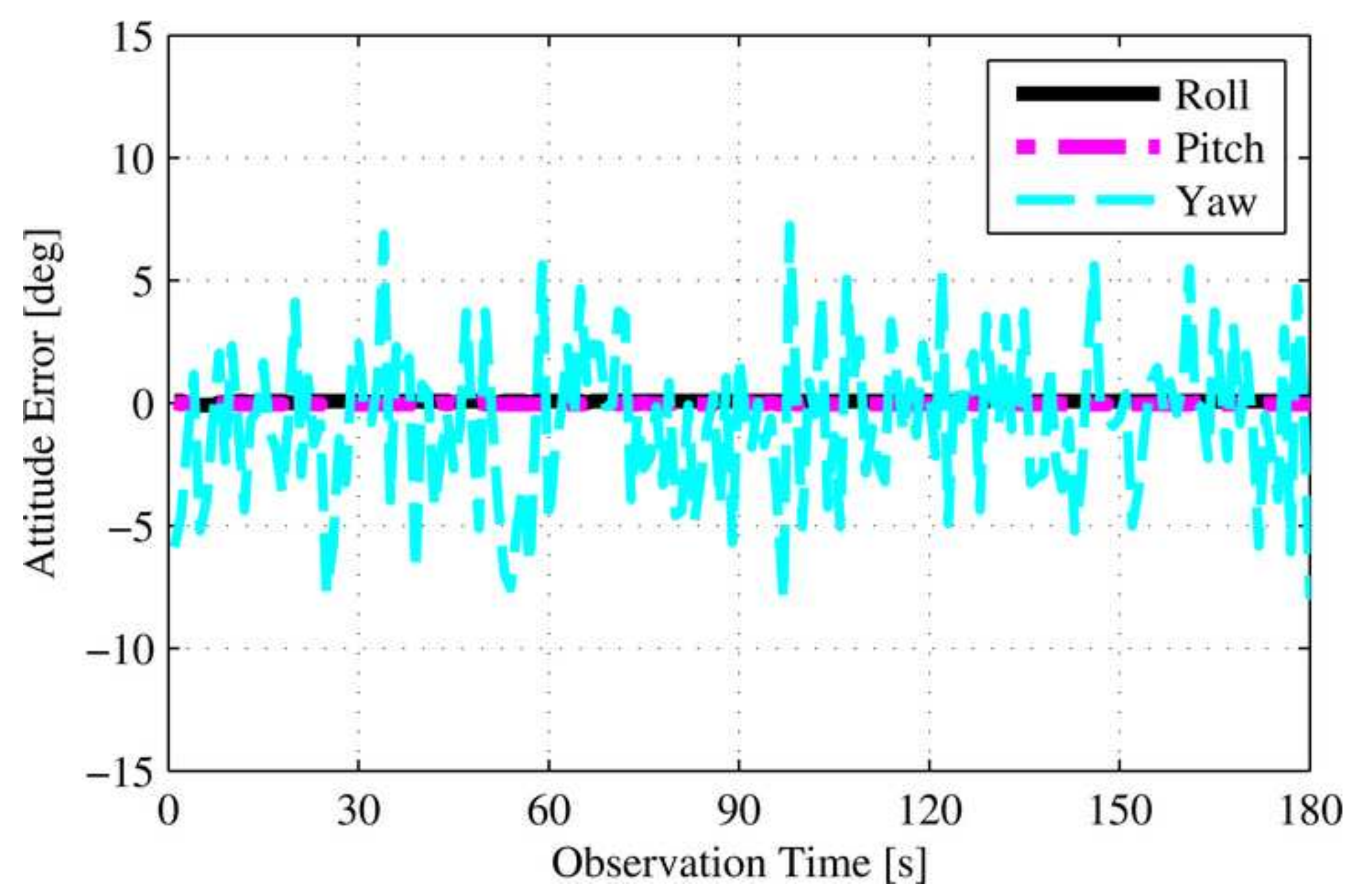




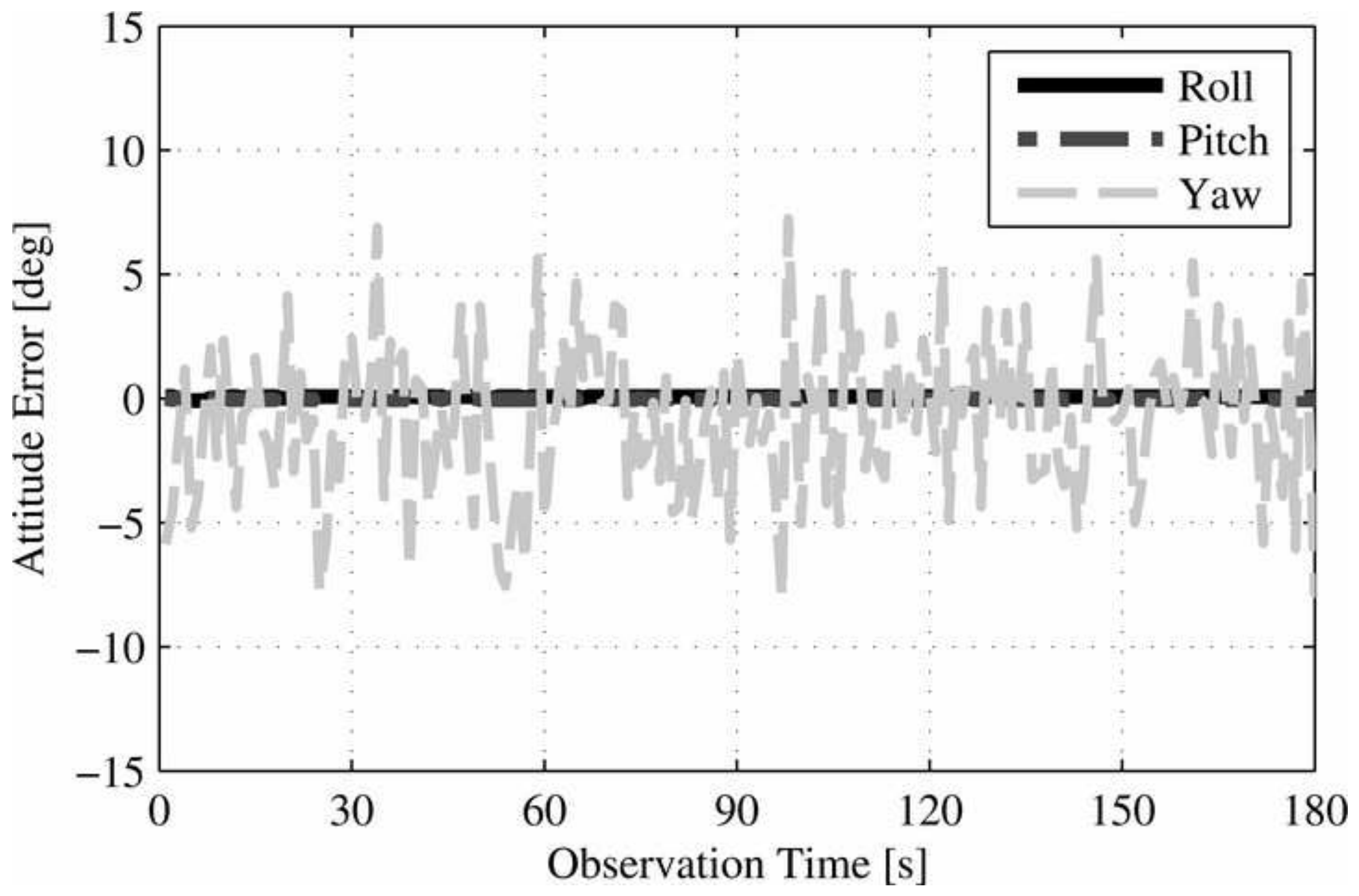




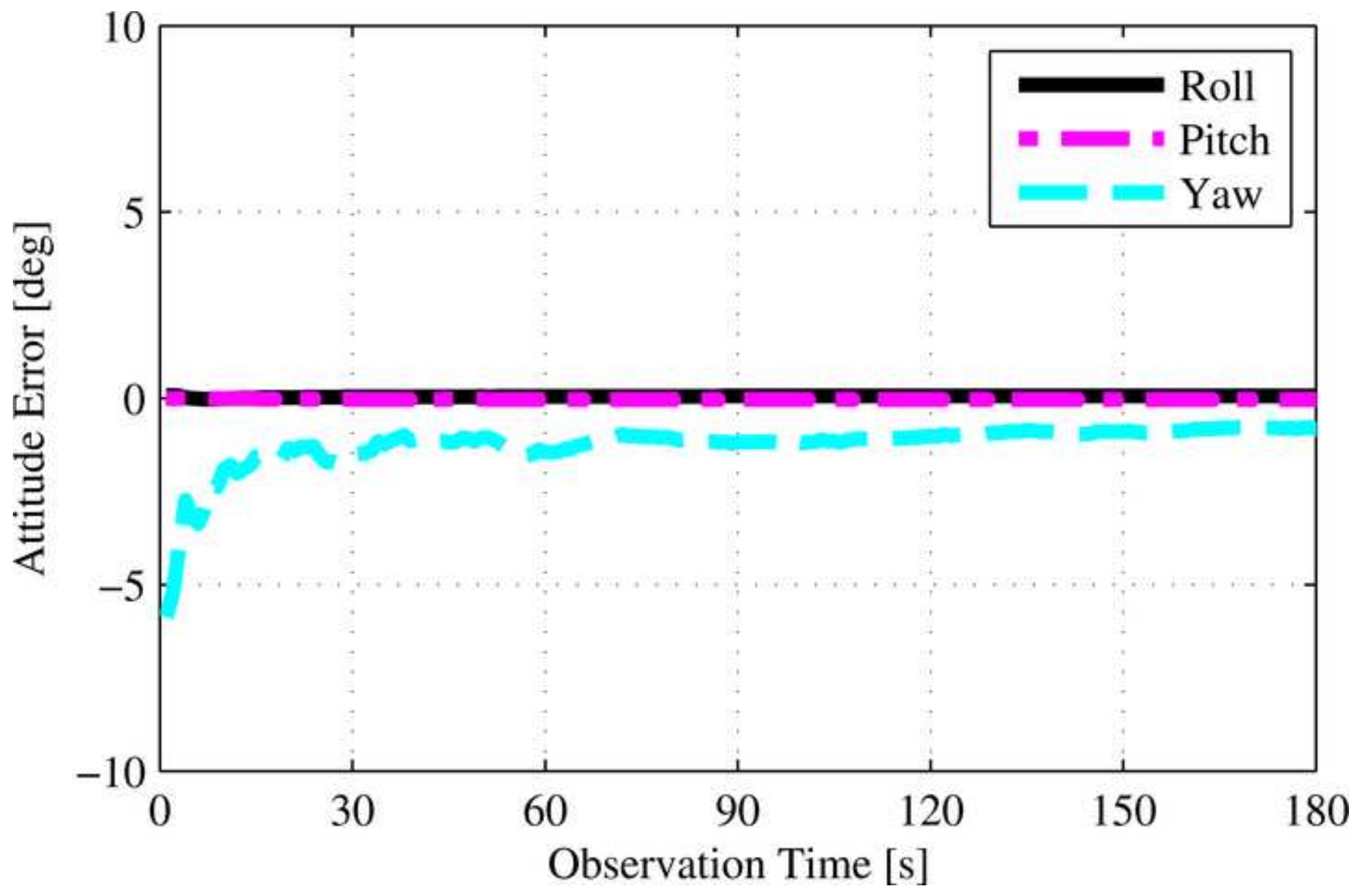




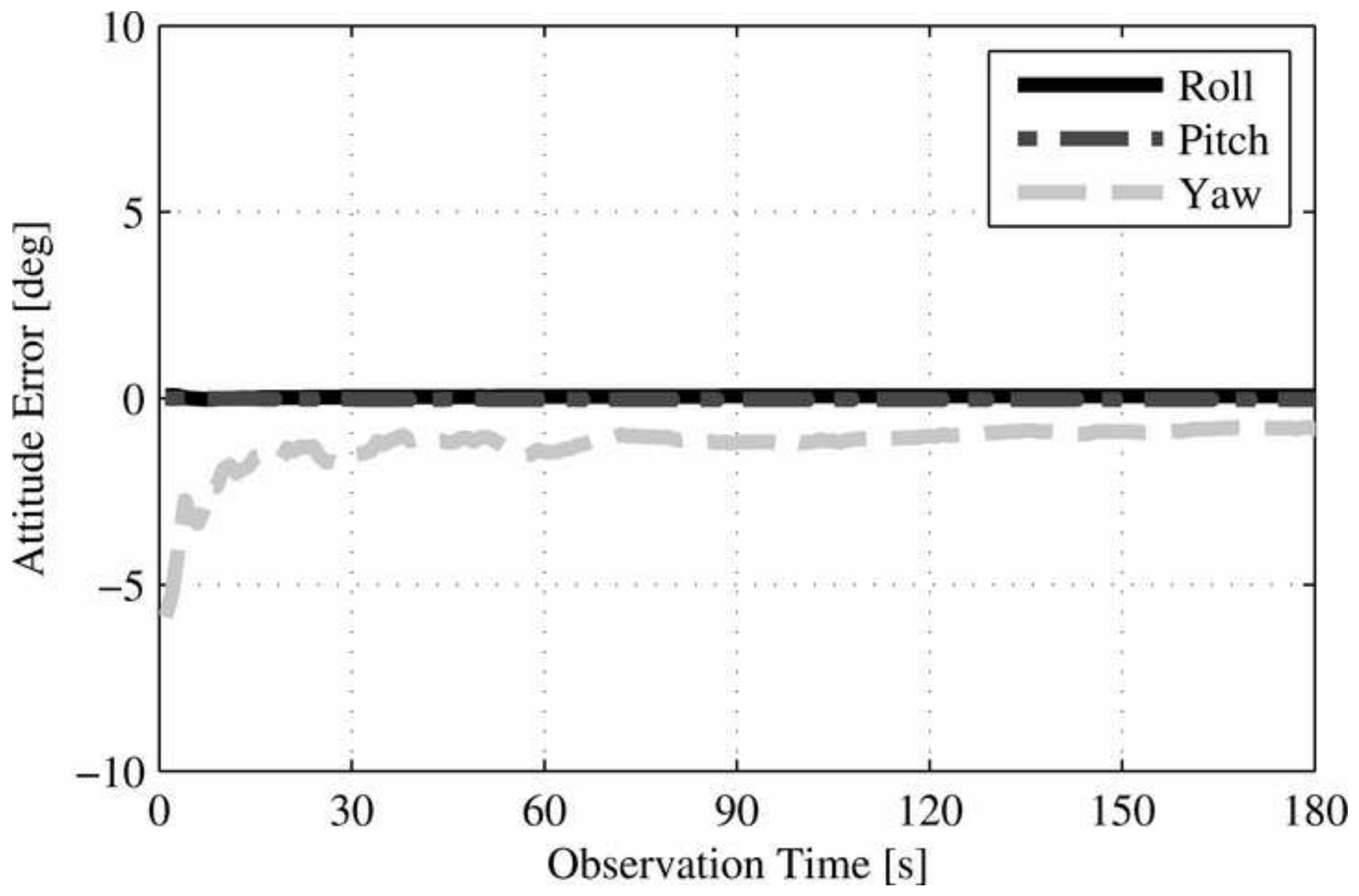




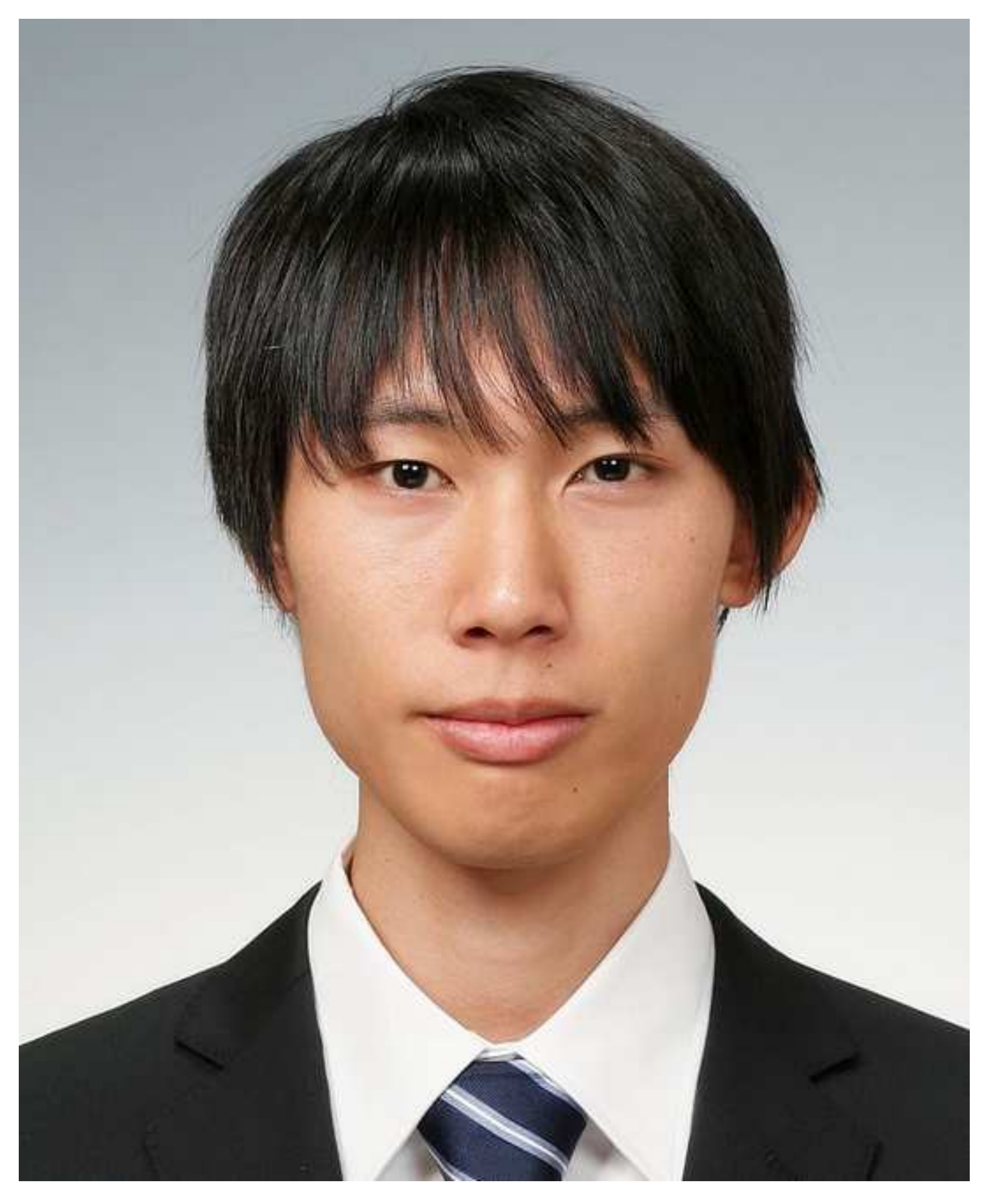 \\ Vitae (Takayuki Ishida, web)}
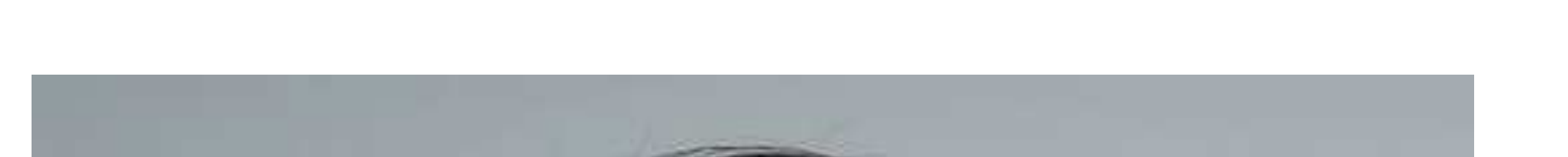

(2)

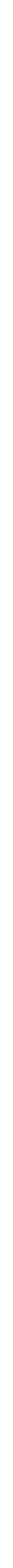




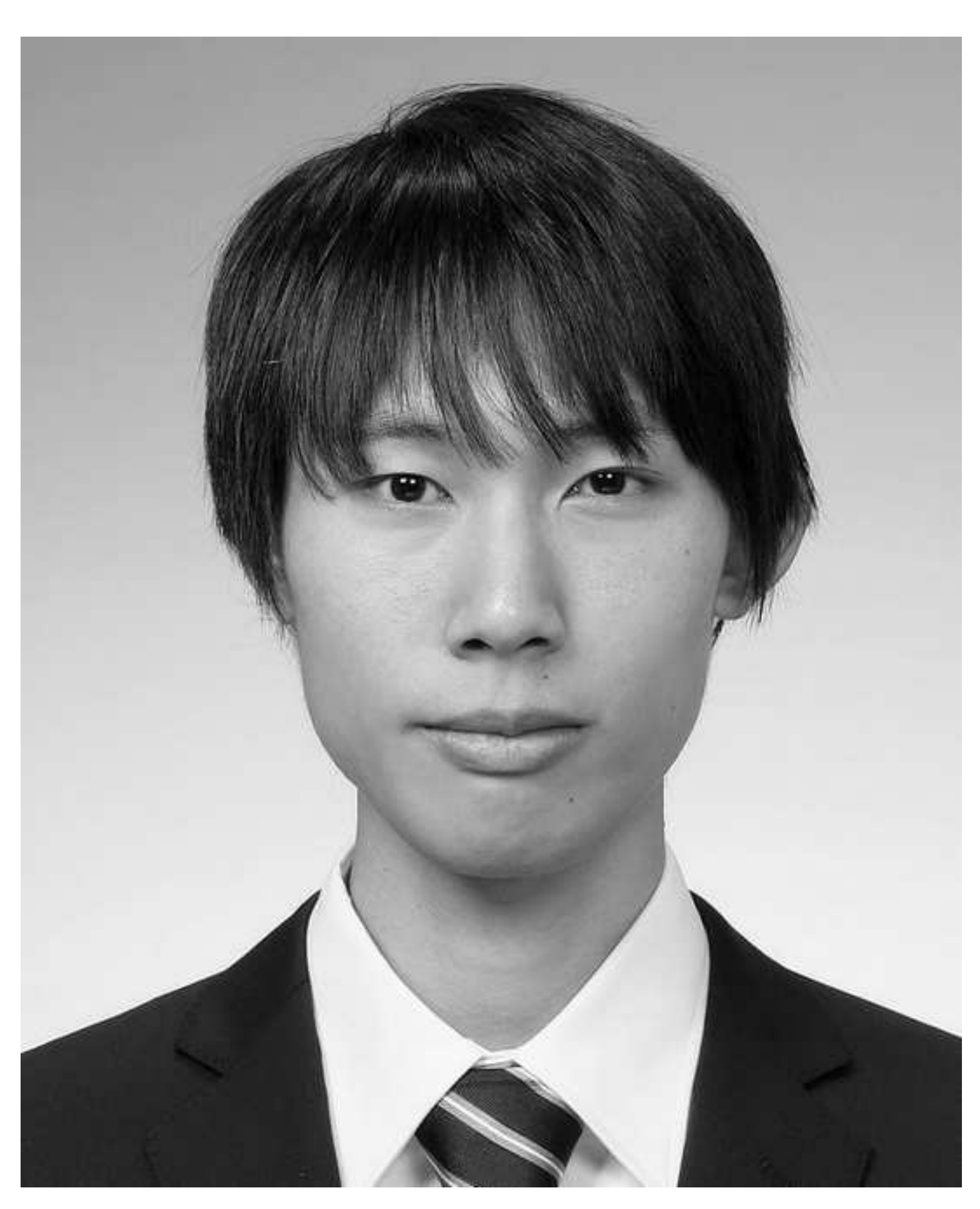 \\ Vitae (Takayuki Ishida, print)}

)

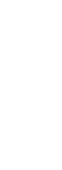
.




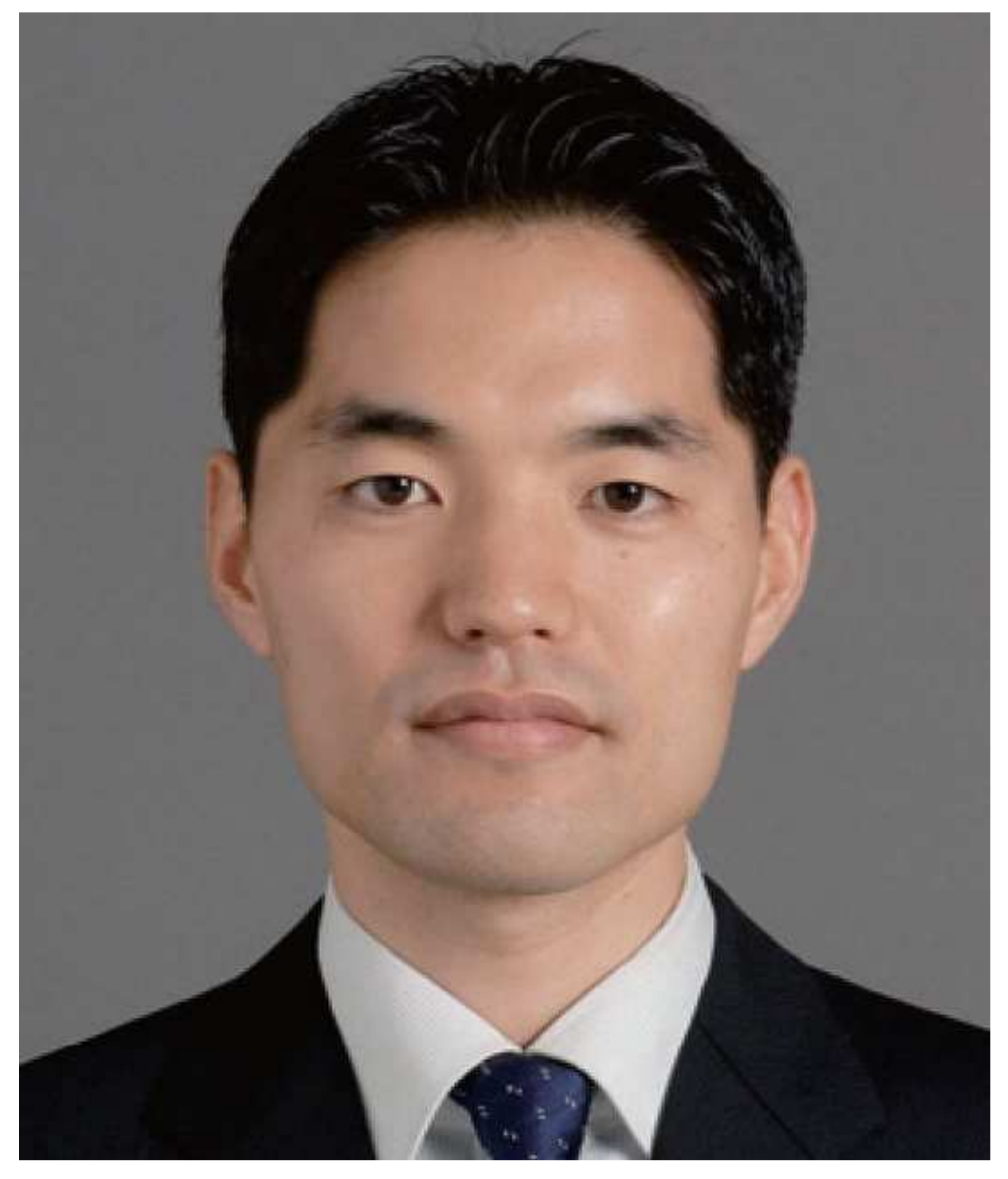

) .

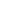

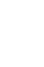
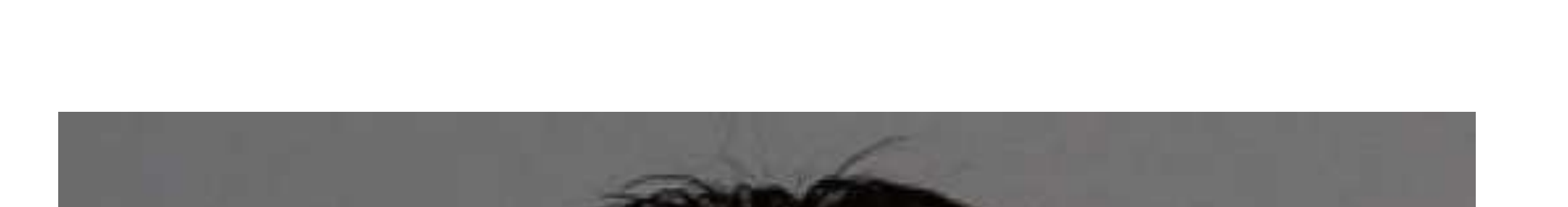


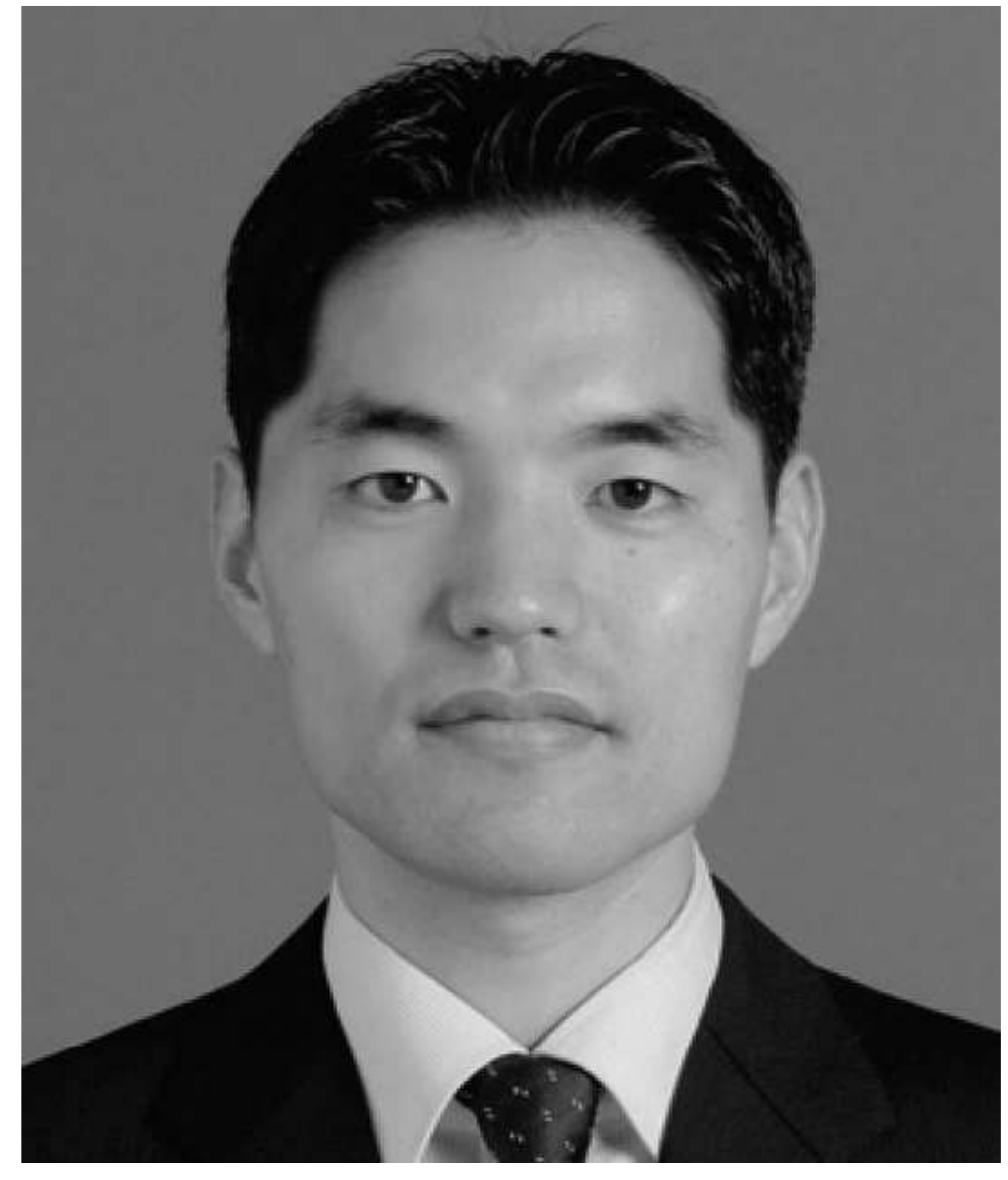

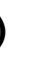

Vitae (Masaki Takahashi, print)
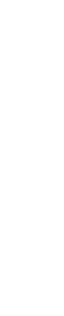